\title{
Tunable $P$-chiral Bisdihydrobenzooxaphosphole Ligands for Enantioselective Hydroformylation
}

Renchang Tan ${ }^{\mathrm{a}}$, Xin Zheng, ${ }^{\mathrm{a}}$, Bo Qu ${ }^{\mathrm{b}}$, C. Avery Sader, Keith R. Fandrick, ${ }^{\mathrm{b}}$ Chris H. Senanayake ${ }^{\mathrm{b} *}$ and Xumu Zhanga*

a.Department of Chemistry and Chemical Biology \& Department of Medicinal Chemistry, Rutgers, The State University of New Jersey, Piscataway, New Jersey 08854, USA

${ }^{\mathrm{b}}$ Chemical Development, Boehringer Ingelheim Pharmaceuticals, Inc., 900 Ridgebury Road, Ridgefield, CT 06877, USA

\section{Supporting Information}

\section{Table of Contents}

1. General Remarks

2. General Procedure for the Asymmetric Hydroformylation

3. GC and HPLC analysis of the chiral aldehydes

S2-S16

4. ${ }^{1} \mathrm{H}$ NMR Spectra of Crude AHF Reaction Mixtures (toluene as solvent) S17-S30

5. Transition State Calculations S31-S53

6. References S54 


\section{General Remarks}

Commercially available reagents were used without further purification. The BIBOP ligands were synthesized according to literature procedures. ${ }^{1} \mathrm{~N}$-allylamides were synthesized following previously reported procedures, ${ }^{2}$ and all characterization data were consistent with those reported for these compounds. All asymmetric hydroformylation reactions were performed in an inert atmosphere using standard Schlenk techniques. Enantiomeric excesses were determined by Agilent GC 7890A or Agilent HPLC 1200 series.

\section{General Procedure for the Asymmetric Hydroformylation}

In a glovebox under nitrogen, ligand $(0.012 \mathrm{mmol})$ and $\left[\mathrm{Rh}(\mathrm{acac})(\mathrm{CO})_{2}\right](0.01 \mathrm{mmol}$ in $0.4 \mathrm{~mL}$ toluene $)$ were added to a $2 \mathrm{~mL}$ vial. After stirring for 10 minutes, the substrate $(1.0 \mathrm{mmol})$ and additional solvent were added to bring the total volume of the reaction mixture to $0.5 \mathrm{~mL}$. The vial was transferred into an autoclave and taken out of the glovebox. Hydrogen and carbon monoxide were added sequentially. The reaction mixture was stirred at $60^{\circ} \mathrm{C}$ for 20 hours. The reaction was cooled and the pressure was carefully released in a well-ventilated fume hood. The conversion and branch to linear ratio of this reaction were determined by ${ }^{1} \mathrm{H}$ NMR spectroscopy from the crude reaction mixture. The enantiomeric excess was determined by GC analysis with a Supelco's Beta Dex 225 column or by Chiral HPLC analysis of the corresponding alcohol resulting from the sodium borohydride reduction of the aldehyde.

\section{GC and HPLC analysis of the chiral aldehydes}

References for chiral separation methods are included. Isolated yields, NMR spectral and HRMS data are provided for chiral aldehydes 21 and 23, which are unreported as AHF products.

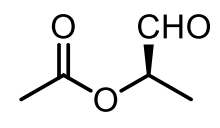

(R)-1-oxopropan-2-yl acetate $(3)^{3}$

Enantiomeric excess was determined by GC with a Supelco's Beta Dex 225 column: $100^{\circ} \mathrm{C}$, stay 5 mins, $4^{\circ} \mathrm{C} / \mathrm{min}$ to $160^{\circ} \mathrm{C}$, stay $5 \mathrm{mins}$, flow rate $=1.0 \mathrm{~mL} / \mathrm{min}, \mathrm{t}_{\text {minor }}=7.6 \mathrm{~min}$, $\mathrm{t}_{\text {major }}=9.3 \mathrm{~min} ; 95.3: 4.7 \mathrm{er}$. 

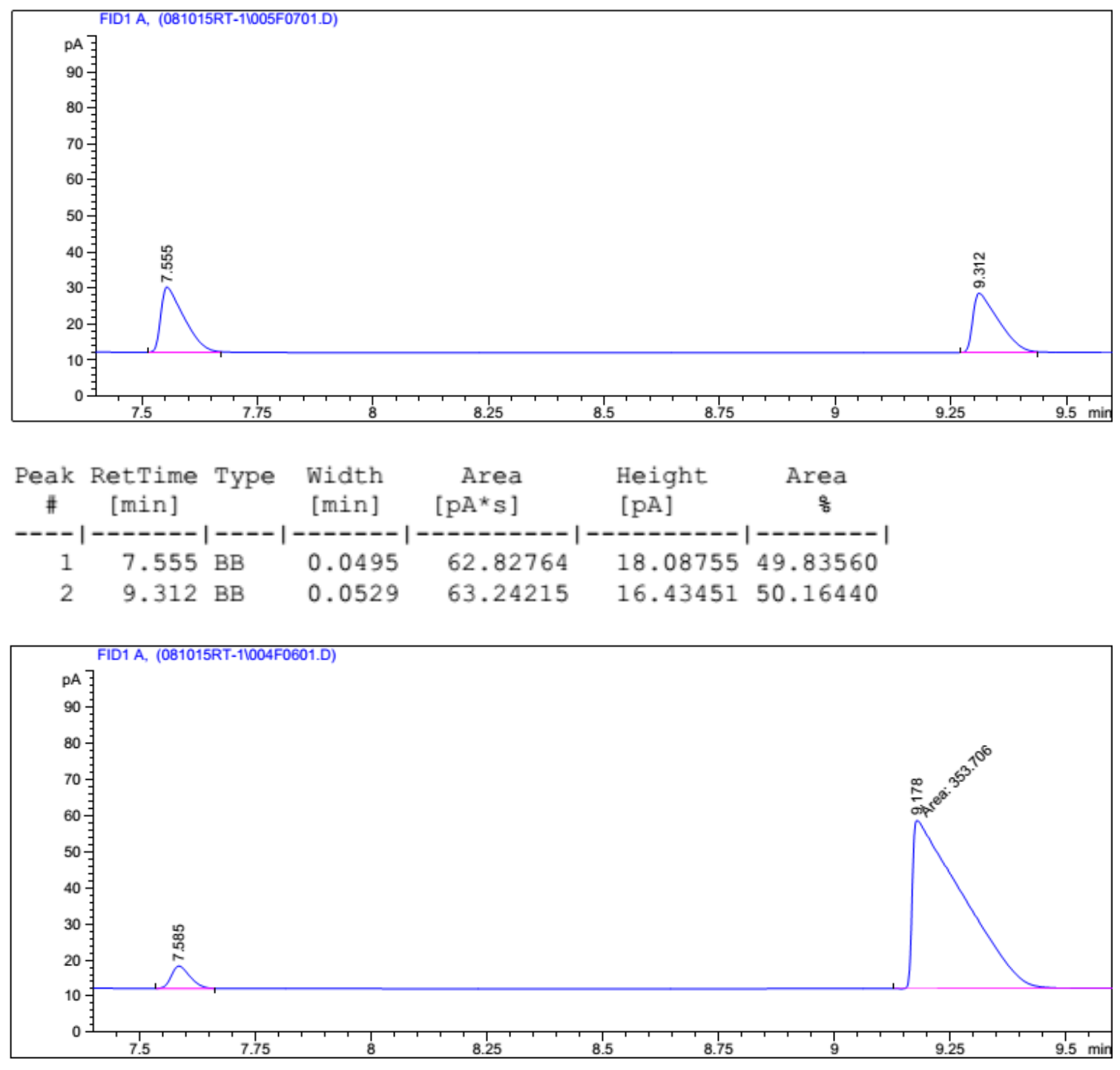

\begin{tabular}{|c|c|c|c|c|c|c|}
\hline $\begin{array}{c}\text { Peak } \\
\#\end{array}$ & $\begin{array}{c}\text { RetTime } \\
\text { [min] }\end{array}$ & Type & $\begin{array}{l}\text { Width } \\
\text { [min] }\end{array}$ & $\begin{array}{r}\text { Area } \\
{\left[\mathrm{pA}^{*} \mathrm{~s}\right]}\end{array}$ & $\begin{array}{l}\text { Height } \\
{[\mathrm{pA}]}\end{array}$ & $\begin{array}{c}\text { Area } \\
\frac{8}{6}\end{array}$ \\
\hline & & & & ----------1 & & \\
\hline $\begin{array}{l}1 \\
2\end{array}$ & $\begin{array}{l}7.585 \\
9.178\end{array}$ & $\begin{array}{l}\text { BB } \\
\text { MM }\end{array}$ & $\begin{array}{l}0.0435 \\
0.1259\end{array}$ & $\begin{array}{r}17.38674 \\
353.70642\end{array}$ & $\begin{array}{r}6.24105 \\
46.83174\end{array}$ & $\begin{array}{r}4.68528 \\
95.31472\end{array}$ \\
\hline
\end{tabular}

Enantiomeric excess was determined by GC with a Supelco's Beta Dex 225 column: $50^{\circ} \mathrm{C}$, stay $5 \mathrm{mins}, 4^{\circ} \mathrm{C} / \mathrm{min}$ to $100^{\circ} \mathrm{C}$, stay $5 \mathrm{mins}$, flow rate $=1.0 \mathrm{~mL} / \mathrm{min}, \mathrm{t}_{\text {minor }}=42.3 \mathrm{~min}, \mathrm{t}_{\text {major }}=43.2 \mathrm{~min} ; 95.2: 4.8 \mathrm{er}$. 

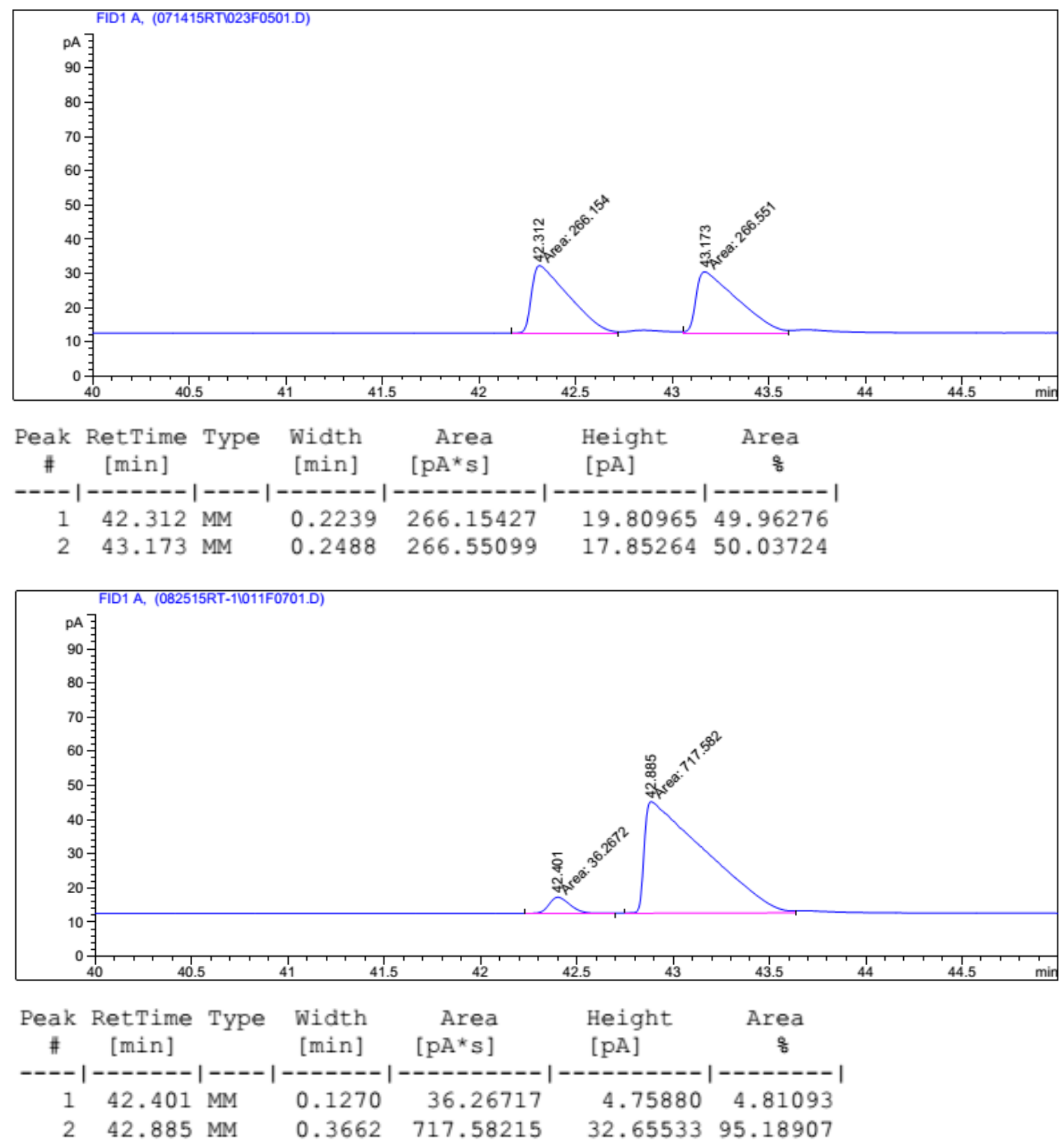

至

$\mathrm{C}_{7} \mathrm{H}_{15} \mathrm{O}_{\mathrm{O}}$ Enantiomeric excess was determined by GC with a Supelco's Beta Dex 225 column: $130^{\circ} \mathrm{C}$, stay $30 \mathrm{mins}$, flow rate $=1.0 \mathrm{~mL} / \mathrm{min}, \mathrm{t}_{\text {minor }}=26.3 \mathrm{~min}, \mathrm{t}_{\text {major }}=27.5 \mathrm{~min} ; 94.5: 5.5 \mathrm{er}$. 

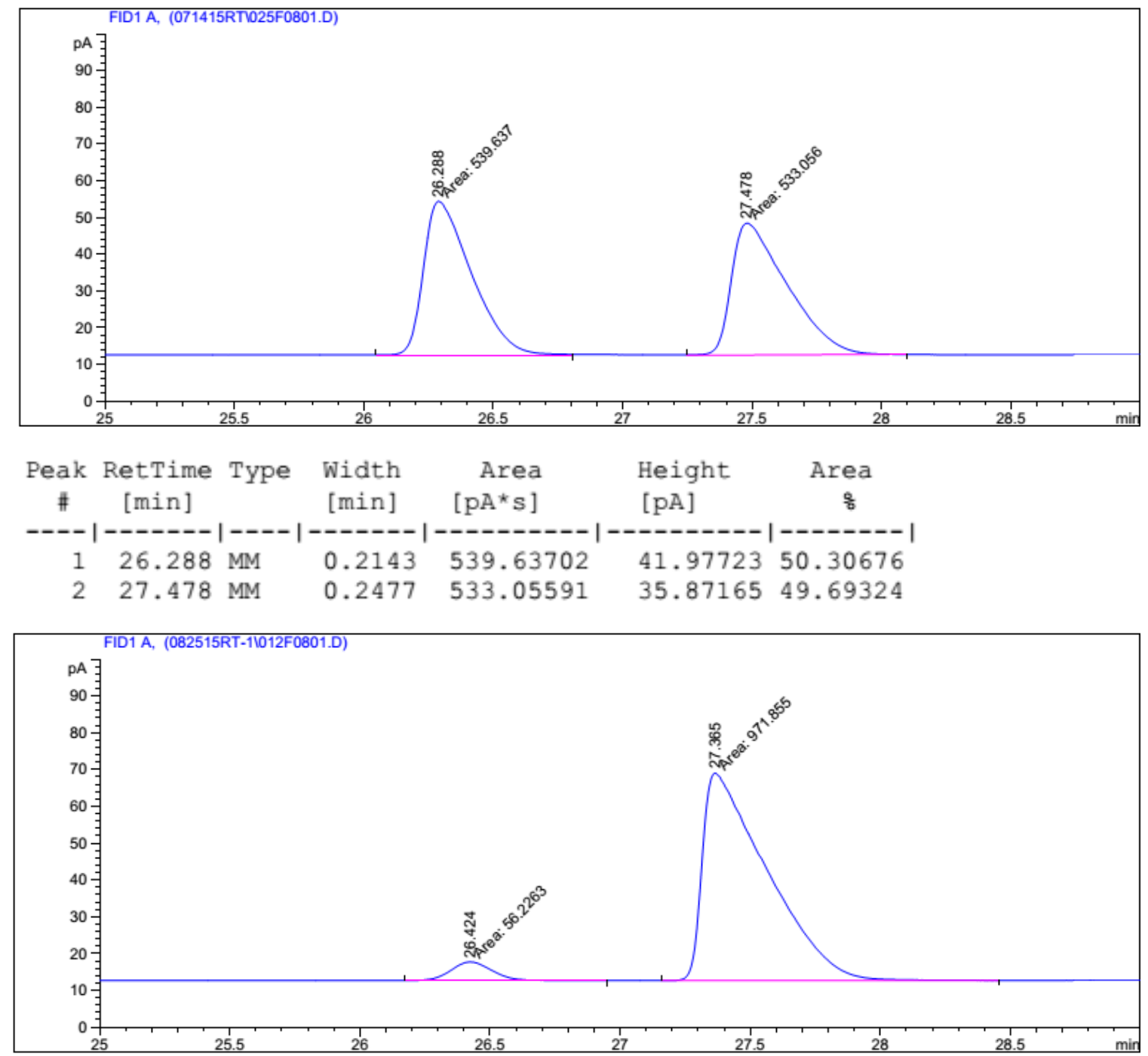

\begin{tabular}{|c|c|c|c|c|c|c|}
\hline $\begin{array}{c}\text { Peak } \\
\#\end{array}$ & $\begin{array}{c}\text { RetTime } \\
\text { [min] }\end{array}$ & Type & $\begin{array}{c}\text { Width } \\
\text { [min] }\end{array}$ & $\begin{array}{r}\text { Area } \\
{\left[\mathrm{pA}^{*} \mathrm{~s}\right]}\end{array}$ & $\begin{array}{l}\text { Height } \\
{[\mathrm{pA}]}\end{array}$ & $\begin{array}{c}\text { Area } \\
\text { 옿 }\end{array}$ \\
\hline & & & & & & \\
\hline 1 & & & & 56. & & 05 \\
\hline 2 & 27.365 & MM & 376 & 971.85498 & 32727 & 3095 \\
\hline
\end{tabular}

لl

$\mathrm{C}_{9} \mathrm{H}_{19} \mathrm{O}$ Enantiomeric excess was determined by GC with a Supelco's Beta Dex 225 column: $130^{\circ} \mathrm{C}$, stay $85 \mathrm{mins}$, flow rate $=1.0 \mathrm{~mL} / \mathrm{min}, \mathrm{t}_{\text {minor }}=71.8 \mathrm{~min}, \mathrm{t}_{\text {major }}=75.3 \mathrm{~min} ; 94.4: 5.6 \mathrm{er}$. 

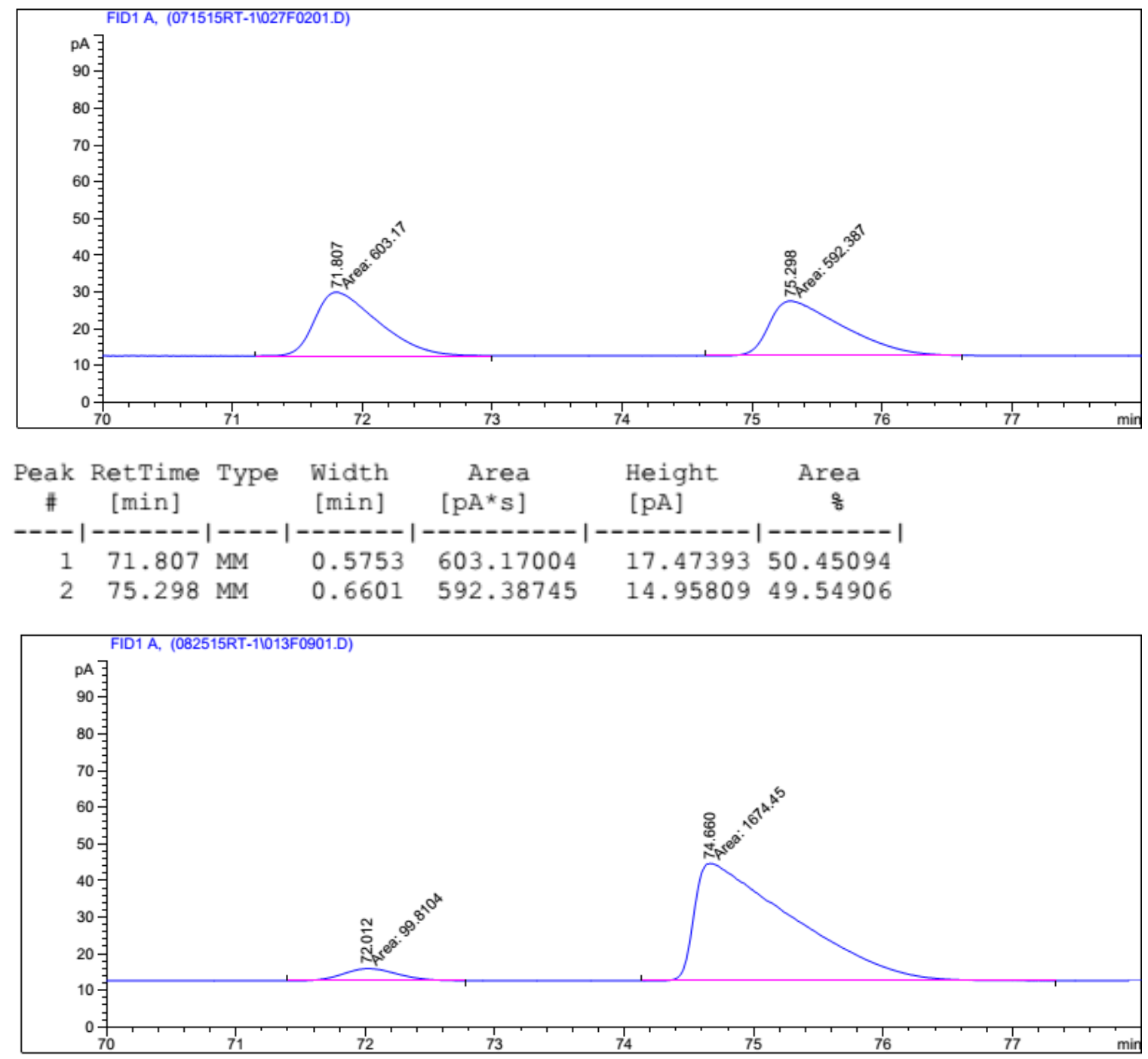

\begin{tabular}{|c|c|c|c|c|c|c|}
\hline $\begin{array}{c}\text { Peak } \\
\#\end{array}$ & $\begin{array}{l}\text { RetTime } \\
\text { [min] }\end{array}$ & Type & $\begin{array}{c}\text { Width } \\
\text { [min] }\end{array}$ & $\begin{array}{r}\text { Area } \\
{\left[\mathrm{pA}^{*} \mathrm{~s}\right]}\end{array}$ & $\begin{array}{l}\text { Height } \\
{[\mathrm{pA}]}\end{array}$ & $\begin{array}{c}\text { Area } \\
\text { 옿 }\end{array}$ \\
\hline & & & & 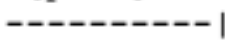 & ---------1 & ---- \\
\hline 1 & 72.01 & MM & & 99.81042 & 3.34903 & 2546 \\
\hline 2 & 74.660 & $M M$ & & 1674.45300 & 7008 & 7454 \\
\hline
\end{tabular}<smiles>CC(C=O)OC(=O)c1ccccc1</smiles>

(R)-1-oxopropan-2-yl benzoate $\left(\begin{array}{ll}1 & 1)^{3}\end{array}\right.$

Enantiomeric excess was determined by GC with a Supelco's Beta Dex 225 column: $130^{\circ} \mathrm{C}$, stay $36 \mathrm{mins}$, flow rate $=1.0 \mathrm{~mL} / \mathrm{min}, \mathrm{t}_{\text {major }}=37.6 \mathrm{~min}, \mathrm{t}_{\mathrm{minor}}=41.3 \mathrm{~min} ; 95.0: 5.0 \mathrm{er}$. 

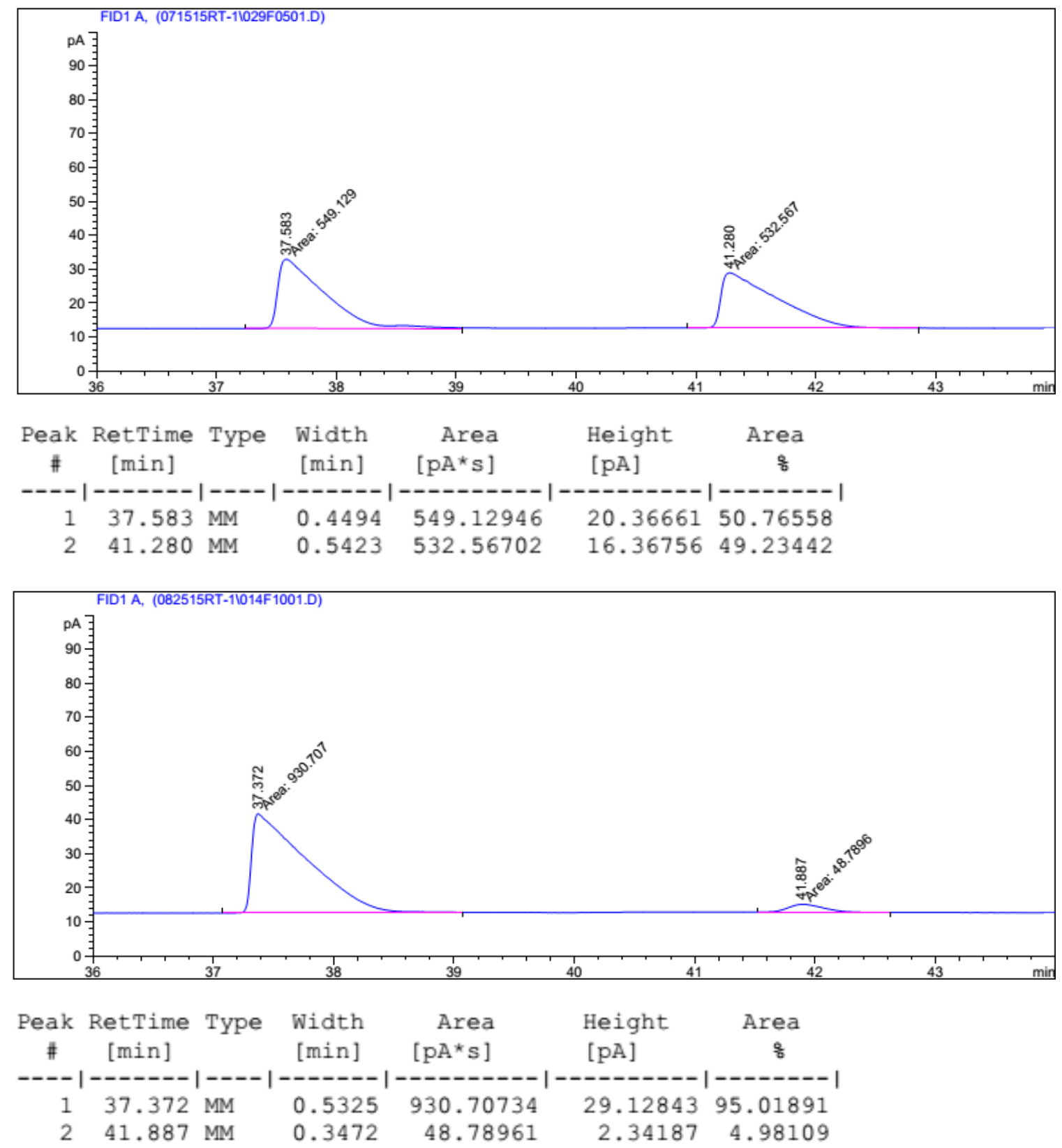

$\mathrm{OHC} \mathrm{CH}_{3} \quad(\boldsymbol{R})$-1,1,1-trifluoro-2-methyl-3-oxopropan-2-yl acetate $(13)^{4}$

$\mathrm{F}_{3} \mathrm{C}$ OAc Enantiomeric excess was determined by GC with a Supelco's Beta Dex 225 column: $100{ }^{\circ} \mathrm{C}$, stay 5 mins, $4^{\circ} \mathrm{C} / \mathrm{min}$ to $160^{\circ} \mathrm{C}$, stay $5 \mathrm{mins}$, flow rate $=1.0 \mathrm{~mL} / \mathrm{min}, \mathrm{t}_{\mathrm{minor}}=3.9 \mathrm{~min}, \mathrm{t}_{\mathrm{major}}=4.2 \mathrm{~min} ; 90.0: 10.0 \mathrm{er}$. 

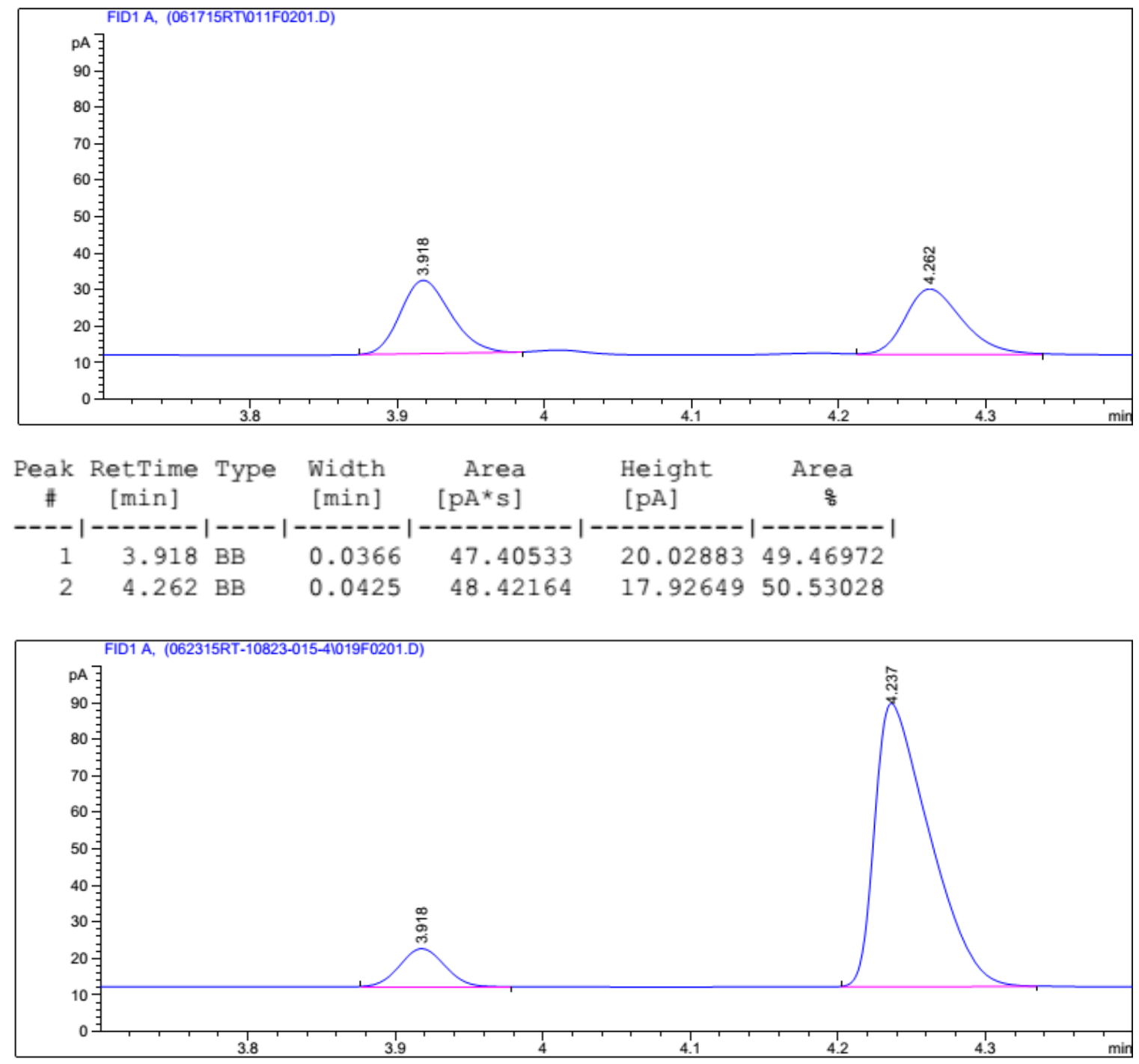

\begin{tabular}{|c|c|c|c|c|c|c|}
\hline $\begin{array}{c}\text { Peak } \\
\#\end{array}$ & $\begin{array}{c}\text { RetTime } \\
\text { [min] }\end{array}$ & Type & $\begin{array}{l}\text { Width } \\
\text { [min] }\end{array}$ & $\begin{array}{r}\text { Area } \\
{\left[\mathrm{pA}^{\star} \mathrm{s}\right]}\end{array}$ & $\begin{array}{l}\text { Height } \\
{[\mathrm{pA}]}\end{array}$ & $\begin{array}{c}\text { Area } \\
\text { 옿 }\end{array}$ \\
\hline & & & & & & \\
\hline 1 & 9 & $3 B$ & & 9601 & & 9. \\
\hline 2 & 4.237 & B & .0391 & 200.30005 & 77.68095 & 02409 \\
\hline
\end{tabular}

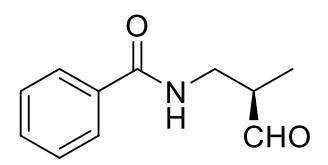

(R)-N-(2-methyl-3-oxopropyl)benzamide $(15)^{3}$

Enantiomeric excess was determined by reducing it into alcohol with $\mathrm{NaBH}_{4}$ and analyzing with HPLC: Daicel Chiralcel AD-3, hexane $/ \mathrm{PrOH}=95: 5$, flow rate $=1.0 \mathrm{~mL} / \mathrm{min}, \lambda=254$ $\mathrm{nm}, \mathrm{t}_{\text {major }}=16.7 \mathrm{~min}, \mathrm{t}_{\text {minor }}=17.6 \mathrm{~min}, 89.2: 10.8 \mathrm{er}$. 

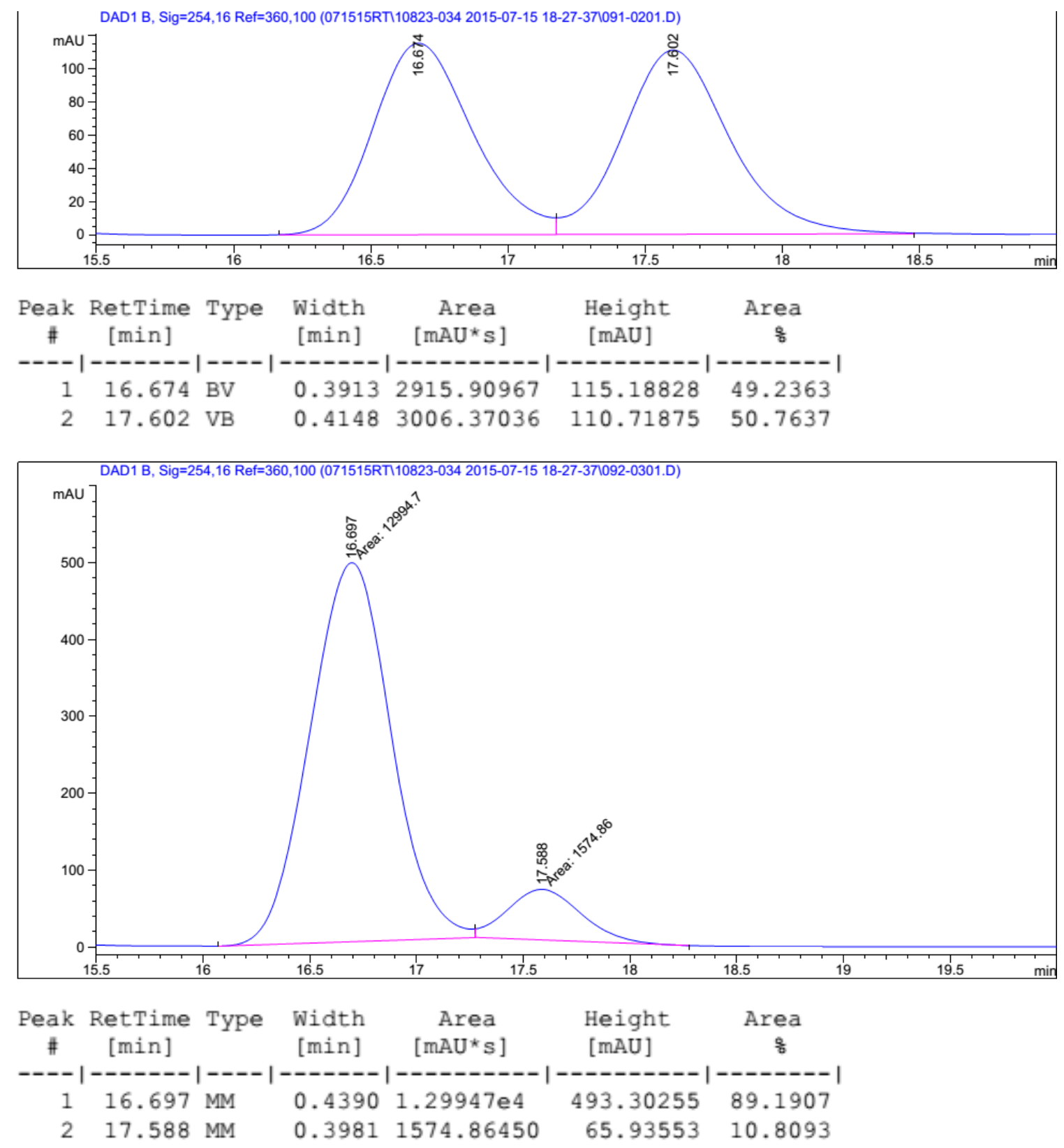
Enantiomeric excess was determined by reducing it into alcohol with $\mathrm{NaBH}_{4}$ and
analyzing with $\mathrm{HPLC}$ : Daicel Chiralcel $\mathrm{AD}-3$, hexane $/ \mathrm{PrOH}=95: 5$, flow rate $=1.0$
$\mathrm{~mL} / \mathrm{min}, \lambda=254 \mathrm{~nm}, \mathrm{t}_{\text {minor }}=16.1 \mathrm{~min}, \mathrm{t}_{\text {major }}=17.6 \mathrm{~min}, 87.3: 12.7 \mathrm{er}$. 


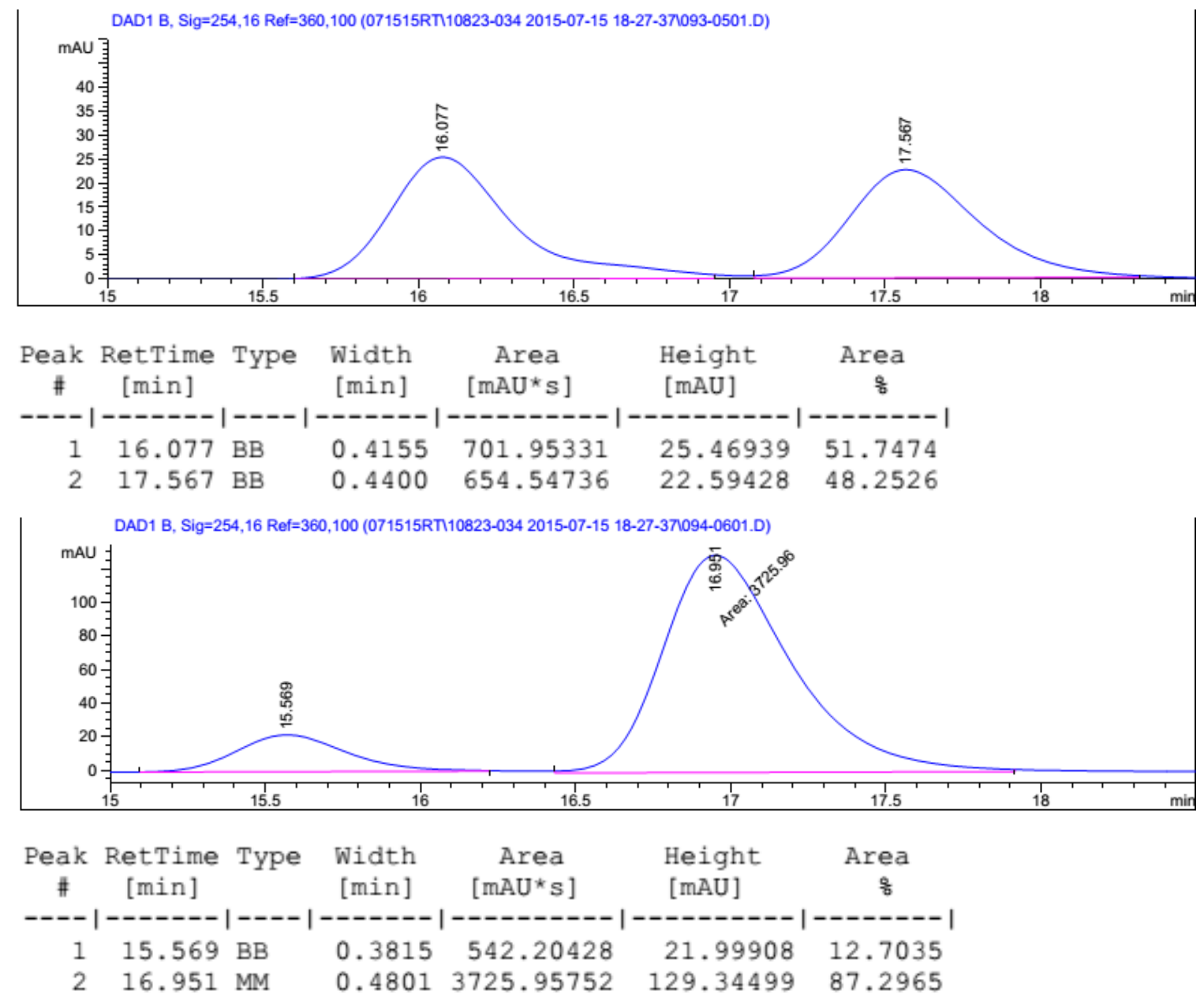

$\overbrace{\mathrm{CHO}}^{\mathrm{O}}$

(R)-4-methyl-N-(2-methyl-3-oxopropyl)benzenesulfonamide $(19)^{3}$

Enantiomeric excess was determined by reducing it into alcohol with $\mathrm{NaBH} 4$ and analyzing with HPLC: Daicel Chiralcel $\mathrm{AD}-\mathrm{H}$, hexane $/ \mathrm{iPrOH}=90: 10$, flowrate $=1.0$ $\mathrm{mL} / \mathrm{min}, \lambda=254 \mathrm{~nm}, \mathrm{t}_{\text {minor }}=32.7 \mathrm{~min}, \mathrm{t}_{\text {major }}=45.6 \mathrm{~min} ; 92.2: 7.8 \mathrm{er}$.

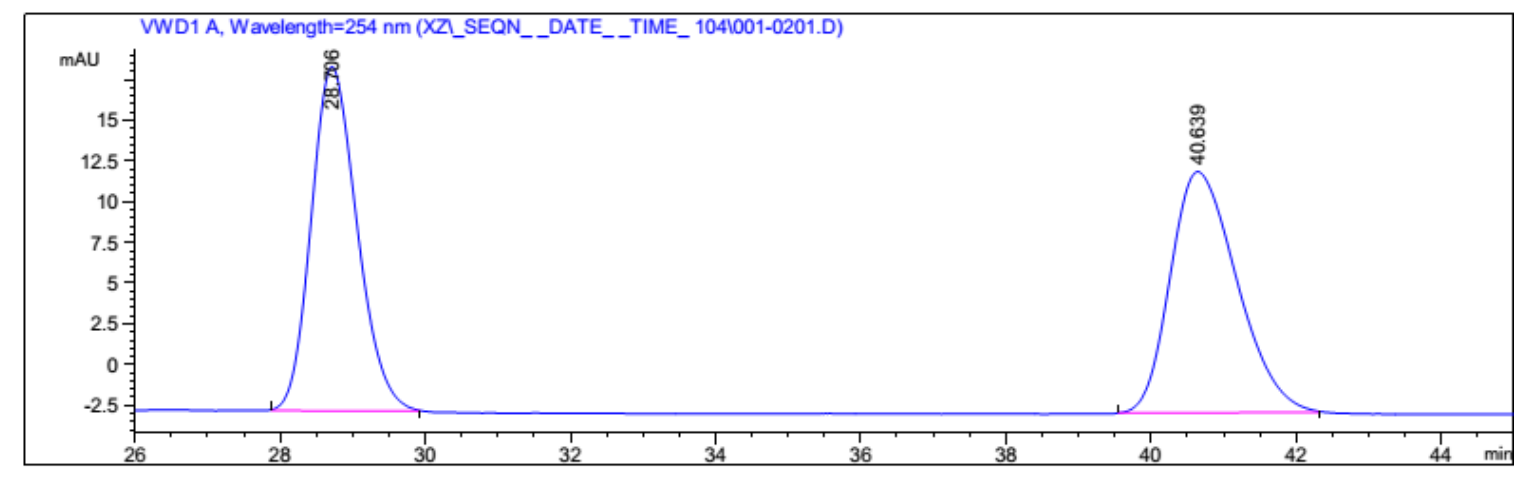



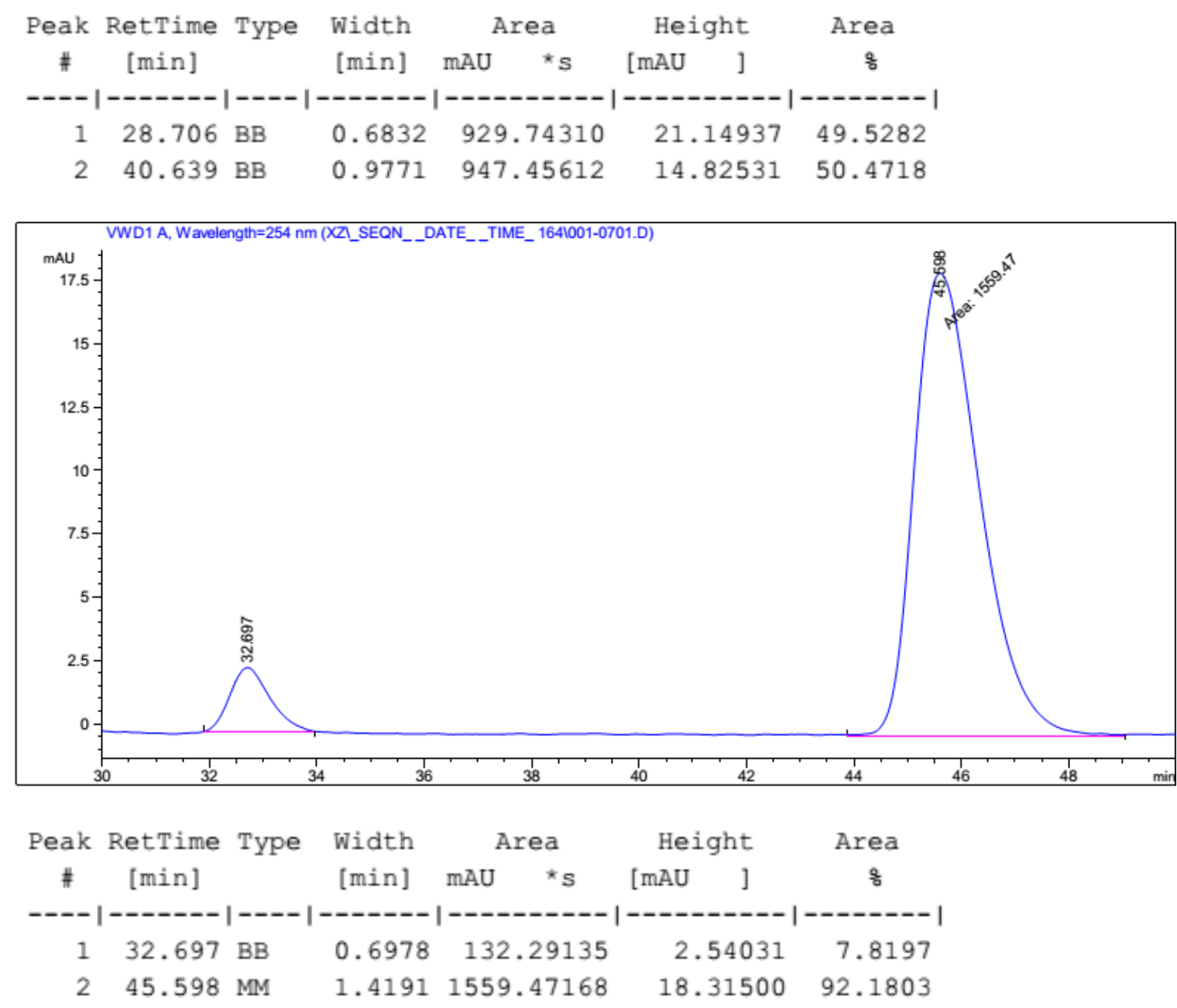

$\underbrace{\mathrm{OMe}}_{\mathrm{CHO}}$

(R)-3-(2-methoxyphenyl)-2-methylpropanal (21) ${ }^{5}$

The crude reaction mixture was purified on silica gel (hexane: ethyl acetate $=19: 1$ ) to afford a clear colorless oil ( $87 \mathrm{mg}, 49 \%$ yield). ${ }^{1} \mathrm{HNMR}\left(400 \mathrm{MHz} \mathrm{CDCl}_{3}\right) \delta 9.68(\mathrm{~d}, J=1.7 \mathrm{~Hz}$, $1 \mathrm{H}), 7.20(\mathrm{td}, J=7.9,1.7 \mathrm{~Hz}, 1 \mathrm{H}), 7.10(\mathrm{dd}, J=7.4,1.6 \mathrm{~Hz}, 1 \mathrm{H}), 6.87(\mathrm{ddd}, J=11.0,8.7,4.6$ $\mathrm{Hz}, 2 \mathrm{H}), 3.80(\mathrm{~s}, 3 \mathrm{H}), 3.07(\mathrm{dd}, J=13.1,6.4 \mathrm{~Hz}, 1 \mathrm{H}), 2.75-2.59(\mathrm{~m}, 2 \mathrm{H}), 1.05(\mathrm{~d}, J=6.9 \mathrm{~Hz}$, $3 \mathrm{H}) .{ }^{13} \mathrm{CNMR}\left(400 \mathrm{MHz}, \mathrm{CDCl}_{3}\right) \delta 205.0,157.4,131.0,127.8,127.1,120.3,110.3,55.1,46.5,31.8,13.3$. All spectral data are in accordance with literature. ${ }^{6} \mathrm{HRMS}(\mathrm{ESI}) \mathrm{m} / \mathrm{z}$ : calc. for $\mathrm{C}_{11} \mathrm{H}_{15} \mathrm{O}_{2}[\mathrm{M}+\mathrm{H}]^{+}$179.10666; found 179.10672 .

Enantiomeric excess was determined by reducing it into alcohol with $\mathrm{NaBH}_{4}$ and analyzing with HPLC: Daicel Chiralcel OD-H, hexane $/ \mathrm{PPOH}=95: 5$, flowrate $=1.0 \mathrm{~mL} / \mathrm{min}, \lambda=220 \mathrm{~nm}, \mathrm{t}_{\text {minor }}=10.6 \mathrm{~min}, \mathrm{t}_{\text {major }}=16.5 \mathrm{~min}$; 93.0:7.0 er. 

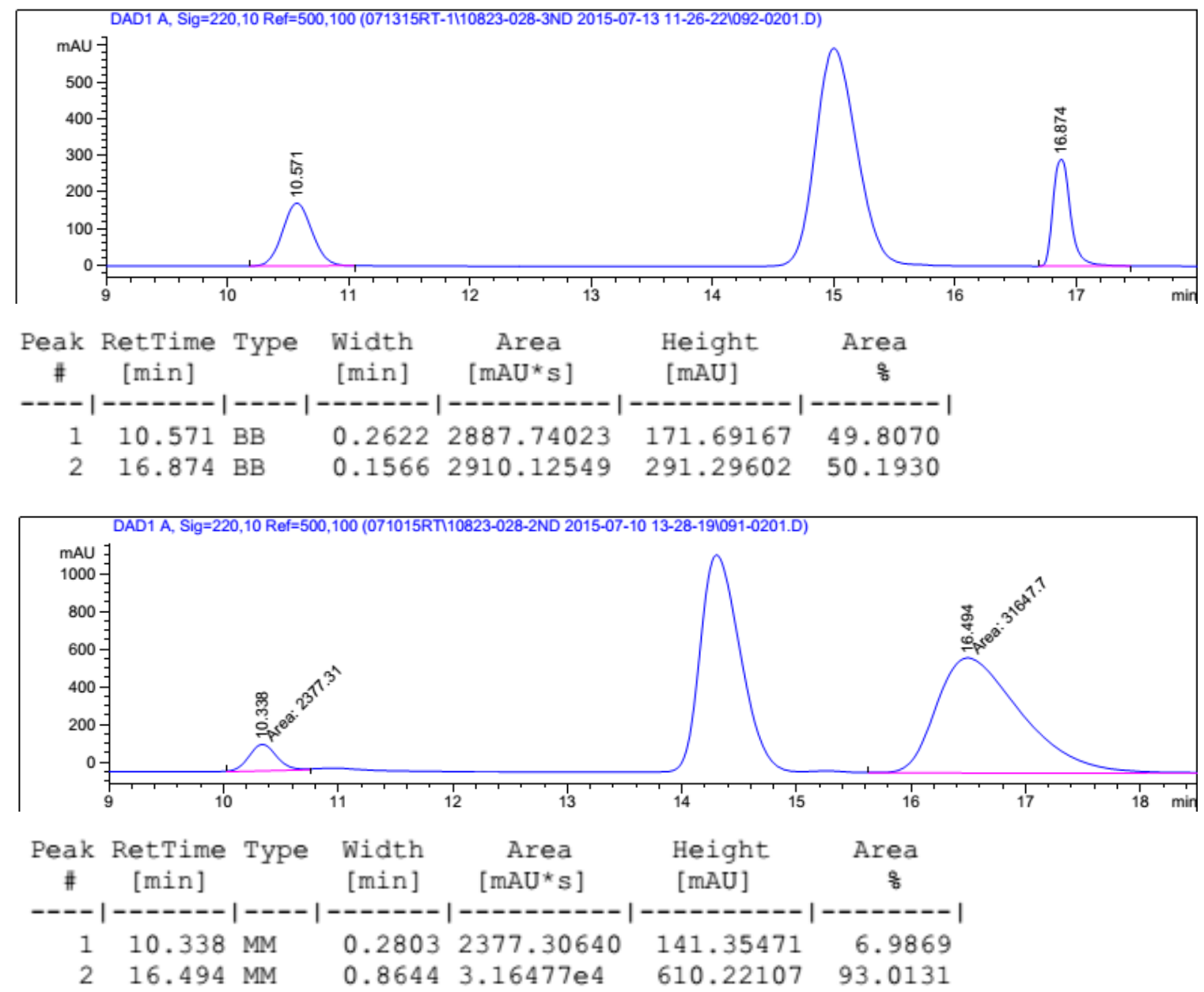

$\overbrace{\mathrm{CHO}}$

(R)-3-(4-chlorophenyl)-2-methylpropanal (23) ${ }^{5}$

The crude reaction mixture was purified on silica gel (hexane: ethyl acetate $=19: 1$ ) to afford a clear pale oil (73 mg, $40 \%$ yield). ${ }^{1} \mathrm{H}$ NMR $\left(400 \mathrm{MHz}, \mathrm{CDCl}_{3}\right) \delta 9.70(\mathrm{~d}, J=1.4$ $\mathrm{Hz}, 1 \mathrm{H}), 7.28$ - $7.24(\mathrm{~m}, 2 \mathrm{H}), 7.13-7.08(\mathrm{~m}, 2 \mathrm{H}), 3.05$ (dd, $J=13.4,5.7 \mathrm{~Hz}, 1 \mathrm{H}), 2.63$ (dddd, $J=26.6,21.5,10.2$, $6.9 \mathrm{~Hz}, 2 \mathrm{H}), 1.09$ (d, $J=6.9 \mathrm{~Hz}, 3 \mathrm{H}) .{ }^{13} \mathrm{C} \mathrm{NMR}\left(400 \mathrm{MHz}, \mathrm{CDCl}_{3}\right) \delta 203.9,137.3,132.2,130.4,128.6,47.9,35.9$, 13.2. HRMS (ESI) $\mathrm{m} / \mathrm{z}$ : calc. for $\mathrm{C}_{10} \mathrm{H}_{15} \mathrm{NOCl}\left[\mathrm{M}+\mathrm{NH}_{4}\right]^{+} 200.08367$; found 200.08376 .

Enantiomeric excess was determined by reducing it into alcohol with $\mathrm{NaBH}_{4}$ and analyzing with HPLC: Daicel Chiralcel OD-H, hexane $/ \mathrm{iPrOH}=95: 5$, flowrate $=1.0 \mathrm{~mL} / \mathrm{min}, \lambda=220 \mathrm{~nm}, \mathrm{t}_{\text {major }}=7.4 \mathrm{~min}, \mathrm{t}_{\text {minor }}=7.9 \mathrm{~min} ; 88.1: 11.9$ er. 

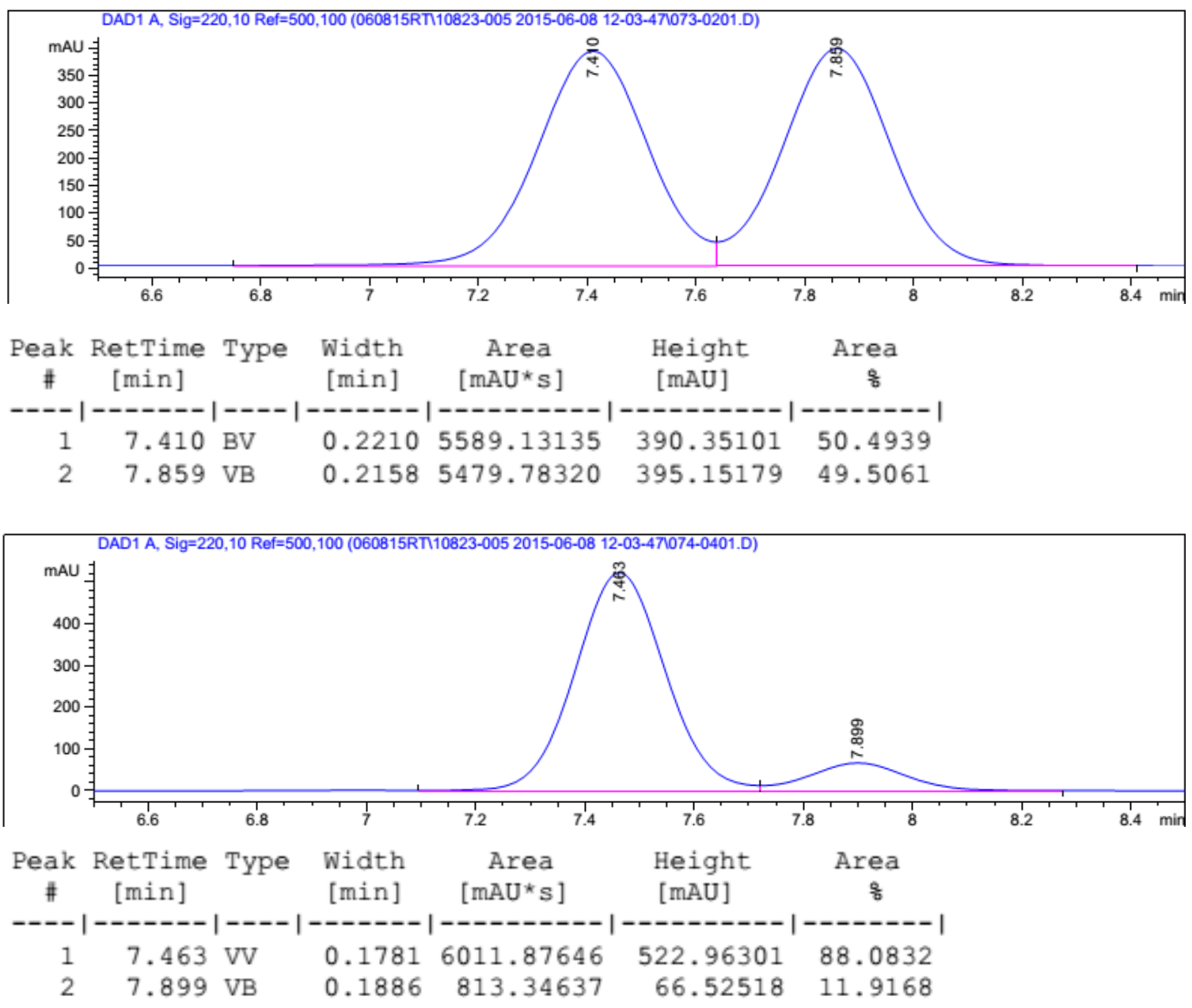

$\mathrm{N}_{\mathrm{CHO}}$

(R)-2-methyl-3-oxopropyl acetate $(25)^{3}$

Enantiomeric excess was determined by GC with a Supelco's Beta Dex 225 column: $90^{\circ} \mathrm{C}$, stay $10 \mathrm{mins}, 0.8^{\circ} \mathrm{C} / \mathrm{min}$ to $100^{\circ} \mathrm{C}$, stay $2 \mathrm{mins}, 0.8^{\circ} \mathrm{C} / \mathrm{min}$ to $110^{\circ} \mathrm{C}$, stay $10 \mathrm{mins}$, flow rate $=1.0$ $\mathrm{mL} / \mathrm{min}, \mathrm{t}_{\text {minor }}=28.0 \mathrm{~min}, \mathrm{t}_{\text {major }}=28.5 \mathrm{~min} ; 93.3: 6.7 \mathrm{er}$.

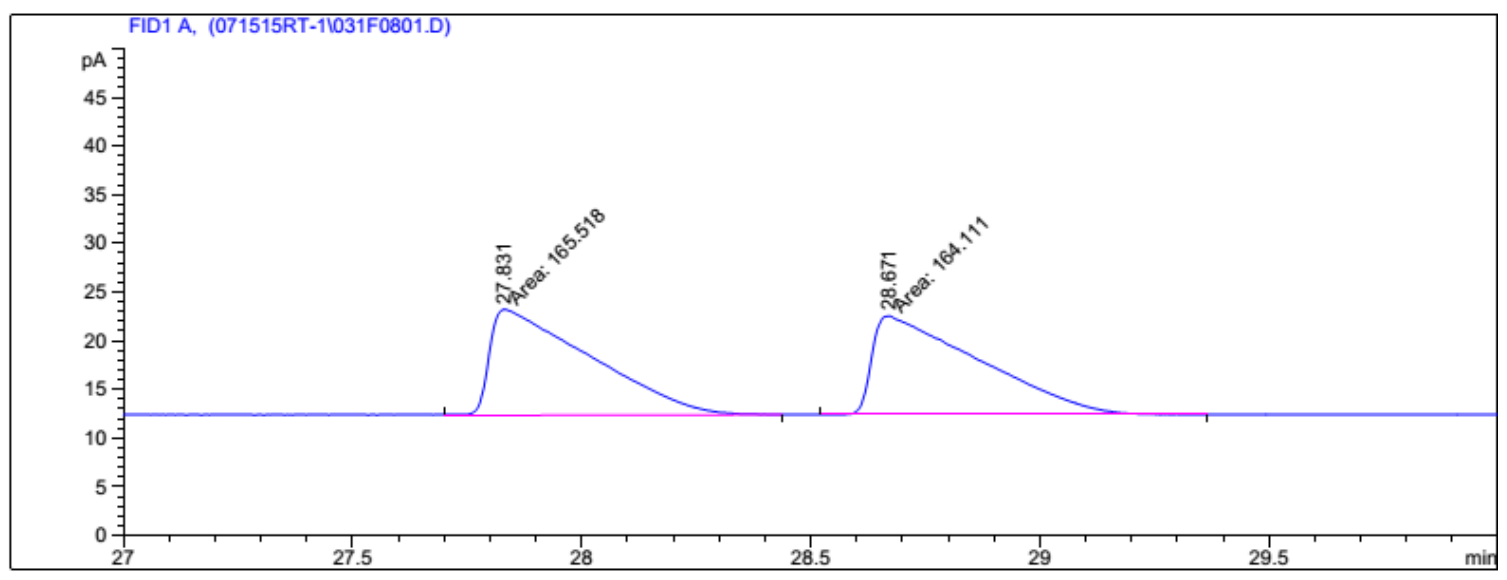



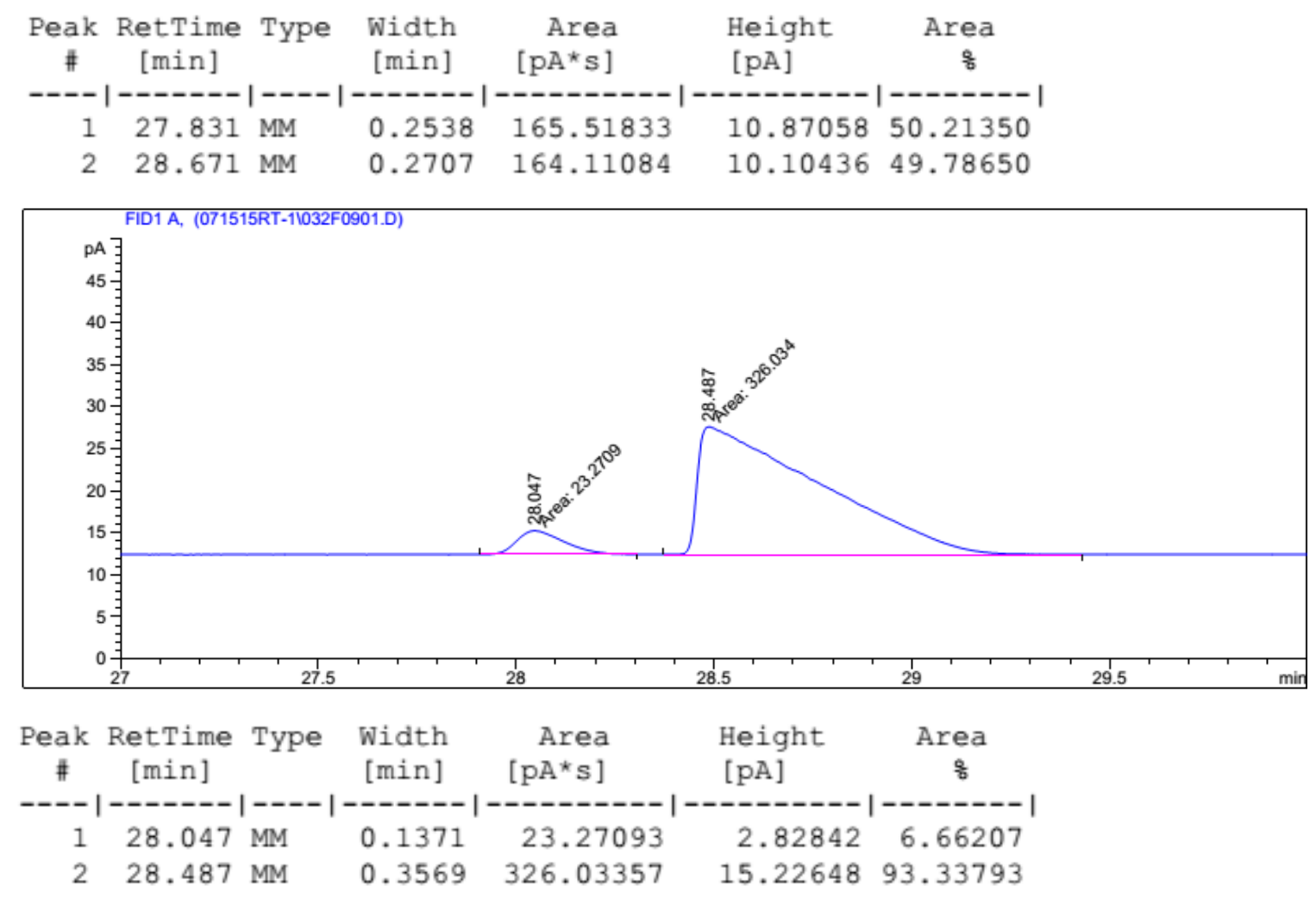

TBS $\bigcirc$ (R)-3-((tert-butyldimethylsilyl)oxy)-2-methylpropanal $(27)^{3}$

CHO Enantiomeric excess was determined by GC with a Supelco's Beta Dex 225 column: $65^{\circ} \mathrm{C}$, stay $30 \mathrm{mins}, 0.7^{\circ} \mathrm{C} / \mathrm{min}$ to $80^{\circ} \mathrm{C}$, stay $10 \mathrm{mins}, 0.7^{\circ} \mathrm{C} / \mathrm{min}$ to $90^{\circ} \mathrm{C}$, stay $15 \mathrm{mins}$, flow rate $=1.0 \mathrm{~mL} / \mathrm{min}, \mathrm{t}_{\text {major }}=70.1$ $\min , \mathrm{t}_{\text {minor }}=70.9 \mathrm{~min} ; 88.8: 11.2 \mathrm{er}$.

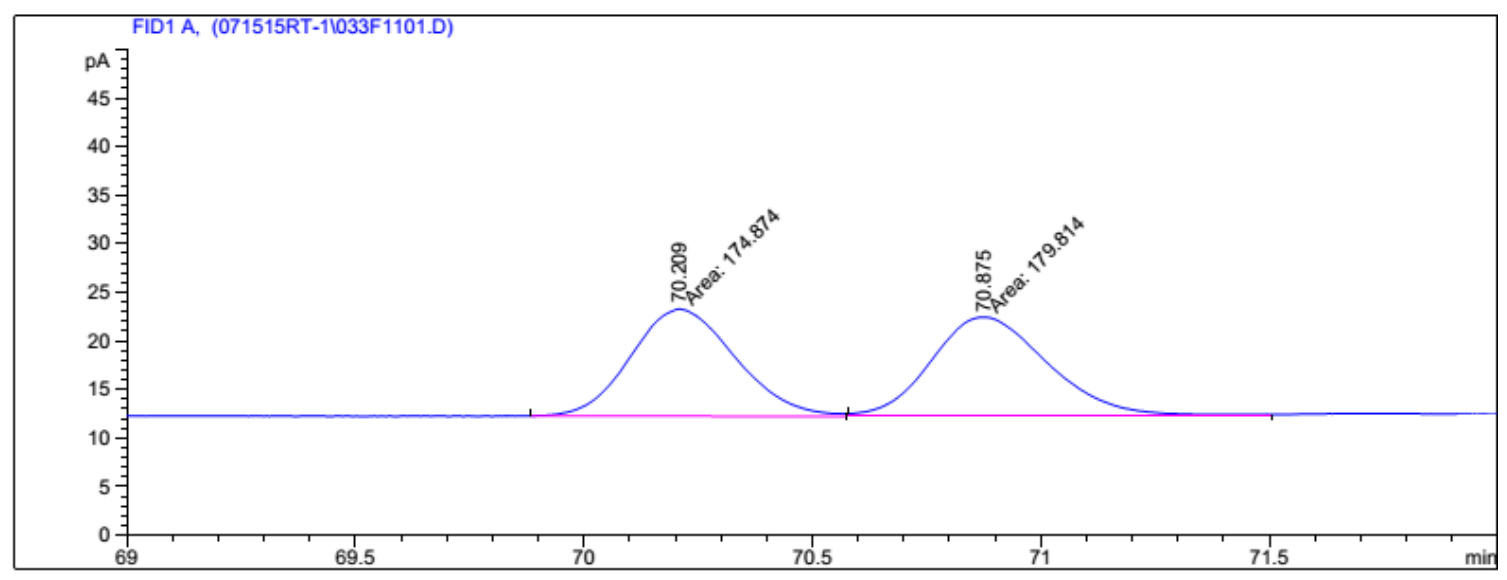



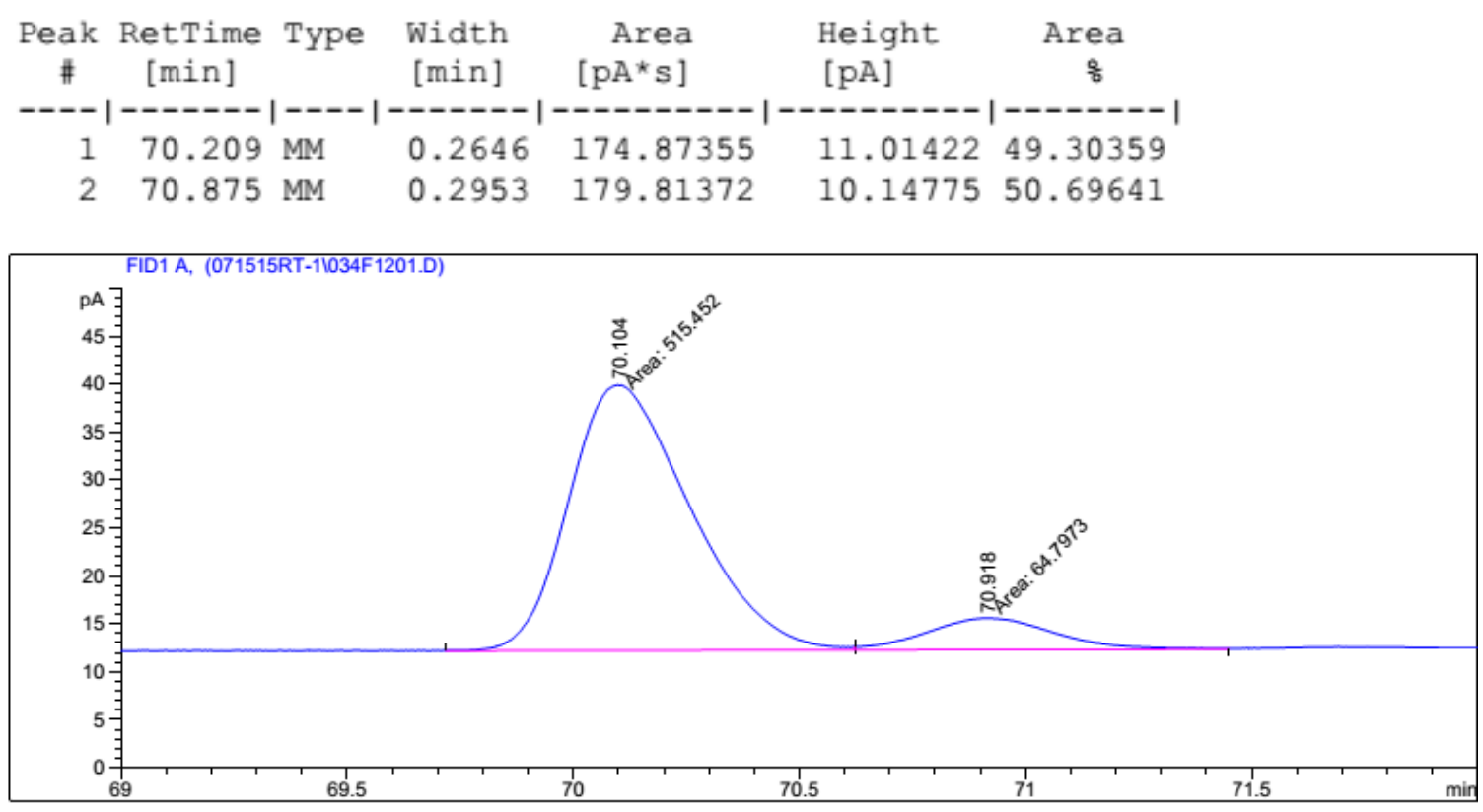

\begin{tabular}{|c|c|c|c|c|c|c|}
\hline $\begin{array}{c}\text { Peak } \\
\#\end{array}$ & $\begin{array}{l}\text { RetTime } \\
\text { [min] }\end{array}$ & Type & $\begin{array}{c}\text { Width } \\
\text { [min] }\end{array}$ & $\begin{array}{r}\text { Area } \\
{\left[\mathrm{pA}^{\star} \mathrm{s}\right]}\end{array}$ & $\begin{array}{l}\text { Height } \\
\text { [pA] }\end{array}$ & $\begin{array}{c}\text { Area } \\
\frac{8}{6}\end{array}$ \\
\hline & & & & 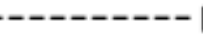 & & \\
\hline 1 & & $\mathrm{MF}$ & & 515.451 & & \\
\hline 2 & 70.918 & FM & .3284 & 64.79728 & 3.28817 & 11.16715 \\
\hline
\end{tabular}

PhO $\left(\right.$ R)-2-methyl-3-phenoxypropanal $(29)^{3}$

Enantiomeric excess was determined by GC with a Supelco's Beta Dex 225 column: $90^{\circ} \mathrm{C}$, stay 0 $\min , 1^{\circ} \mathrm{C} / \mathrm{min}$ to $160^{\circ} \mathrm{C}$, flow rate $=1.0 \mathrm{~mL} / \mathrm{min}, \mathrm{t}_{\text {major }}=49.3 \mathrm{~min}, \mathrm{t}_{\text {minor }}=49.7 \mathrm{~min} ; 86.8: 13.2 \mathrm{er}$.

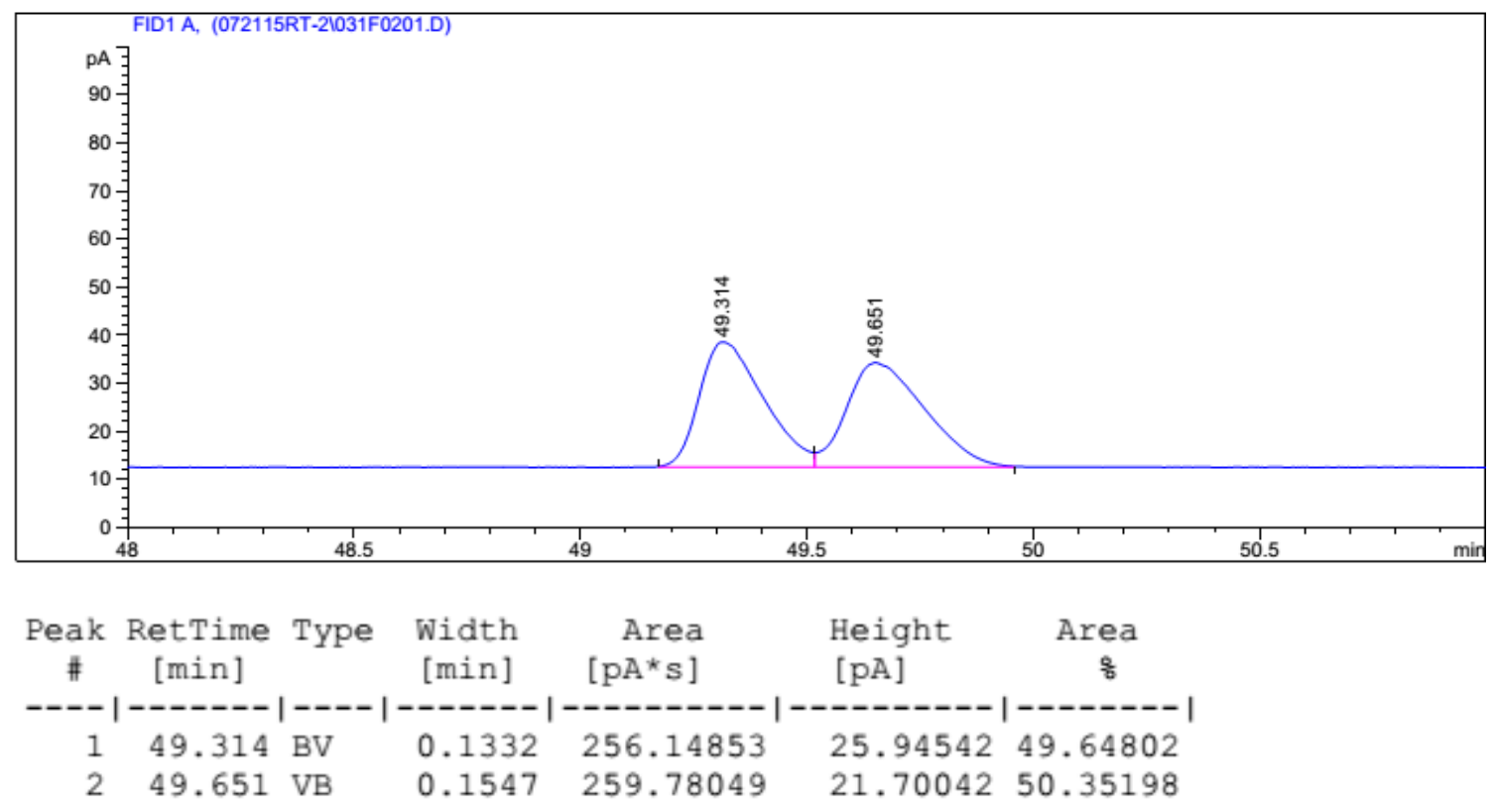




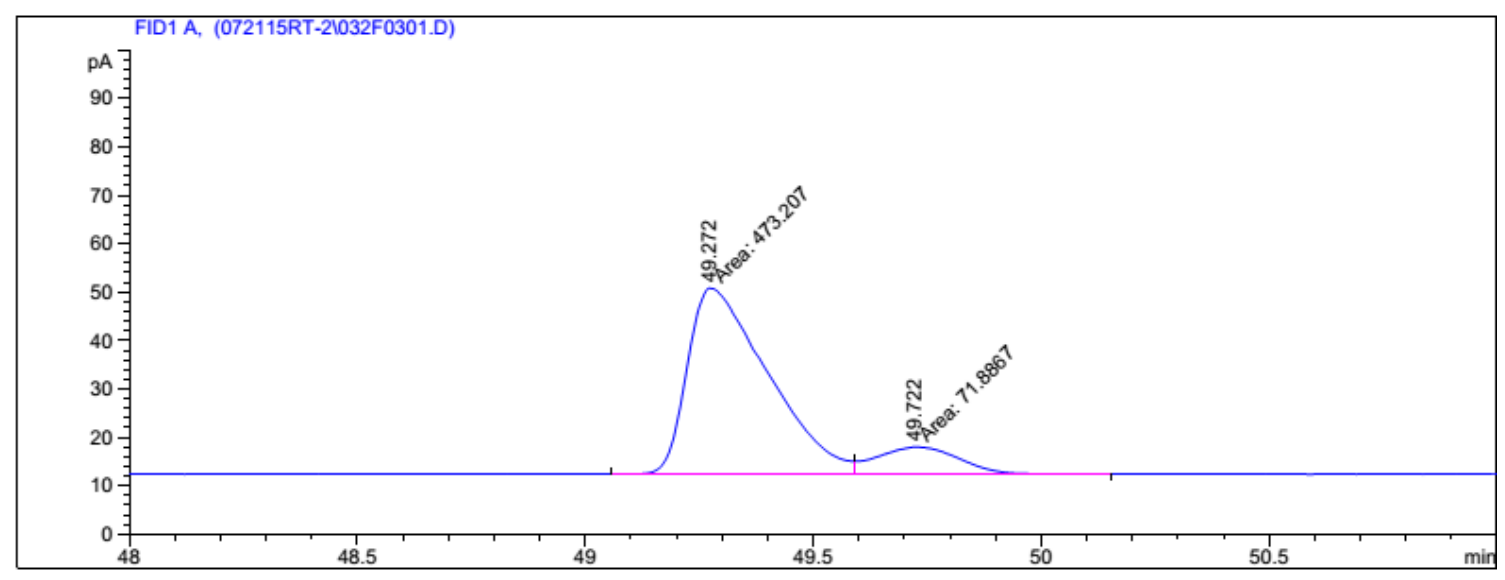

\begin{tabular}{|c|c|c|c|c|c|c|}
\hline $\begin{array}{c}\text { Peak } \\
\#\end{array}$ & $\begin{array}{l}\text { RetTime } \\
\text { [min] }\end{array}$ & Type & $\begin{array}{l}\text { Width } \\
\text { [min] }\end{array}$ & $\begin{array}{r}\text { Area } \\
{\left[\mathrm{pA}^{\star} \mathrm{s}\right]}\end{array}$ & $\begin{array}{l}\text { Height } \\
{[\mathrm{pA}]}\end{array}$ & $\begin{array}{c}\text { Area } \\
\frac{8}{6}\end{array}$ \\
\hline & & & & & & \\
\hline 2 & & & .2114 & 71.88669 & 5.66666 & 13.18796 \\
\hline
\end{tabular}


4. ${ }^{1}$ H NMR Spectra of Crude AHF Reaction Mixtures (toluene as solvent)

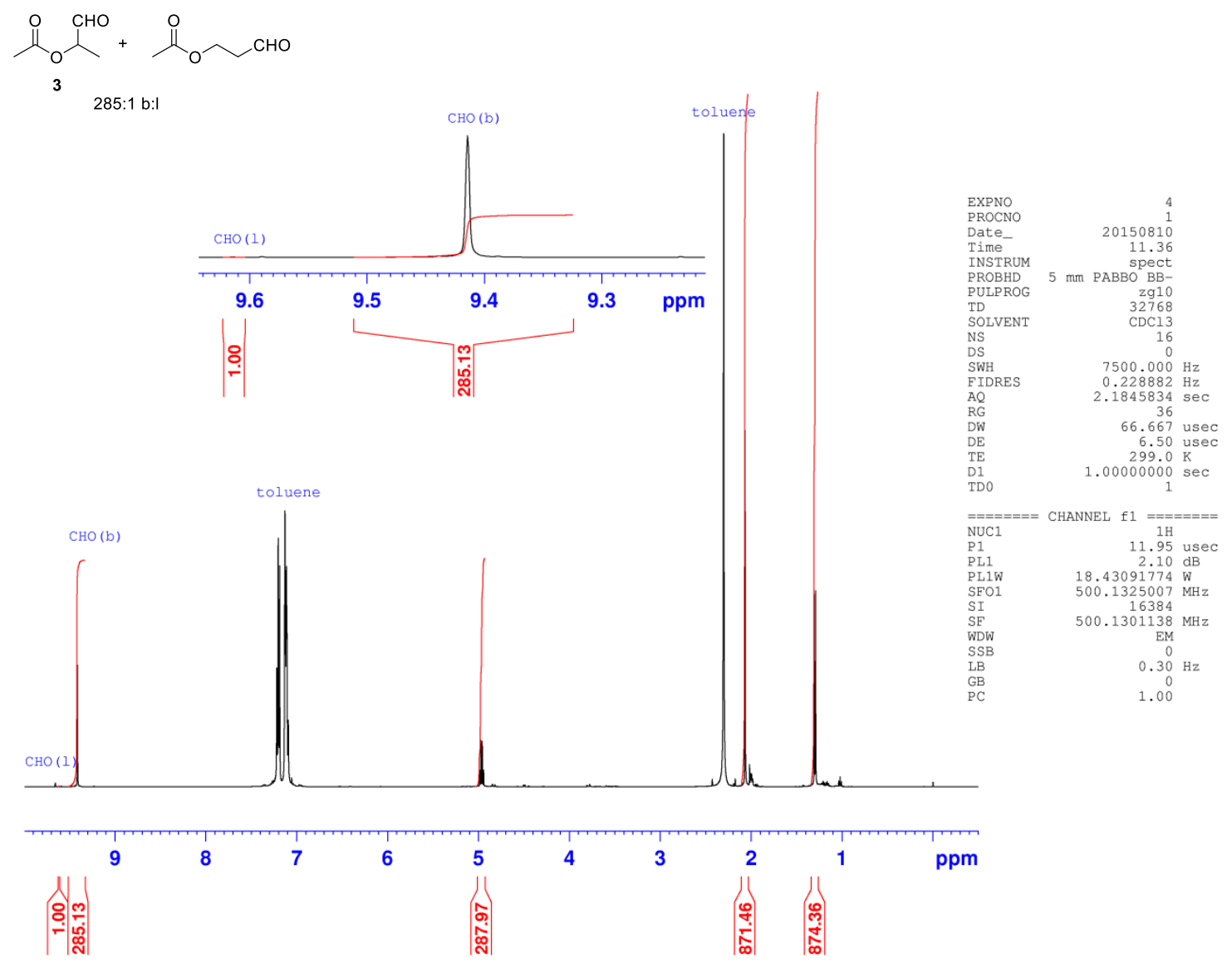




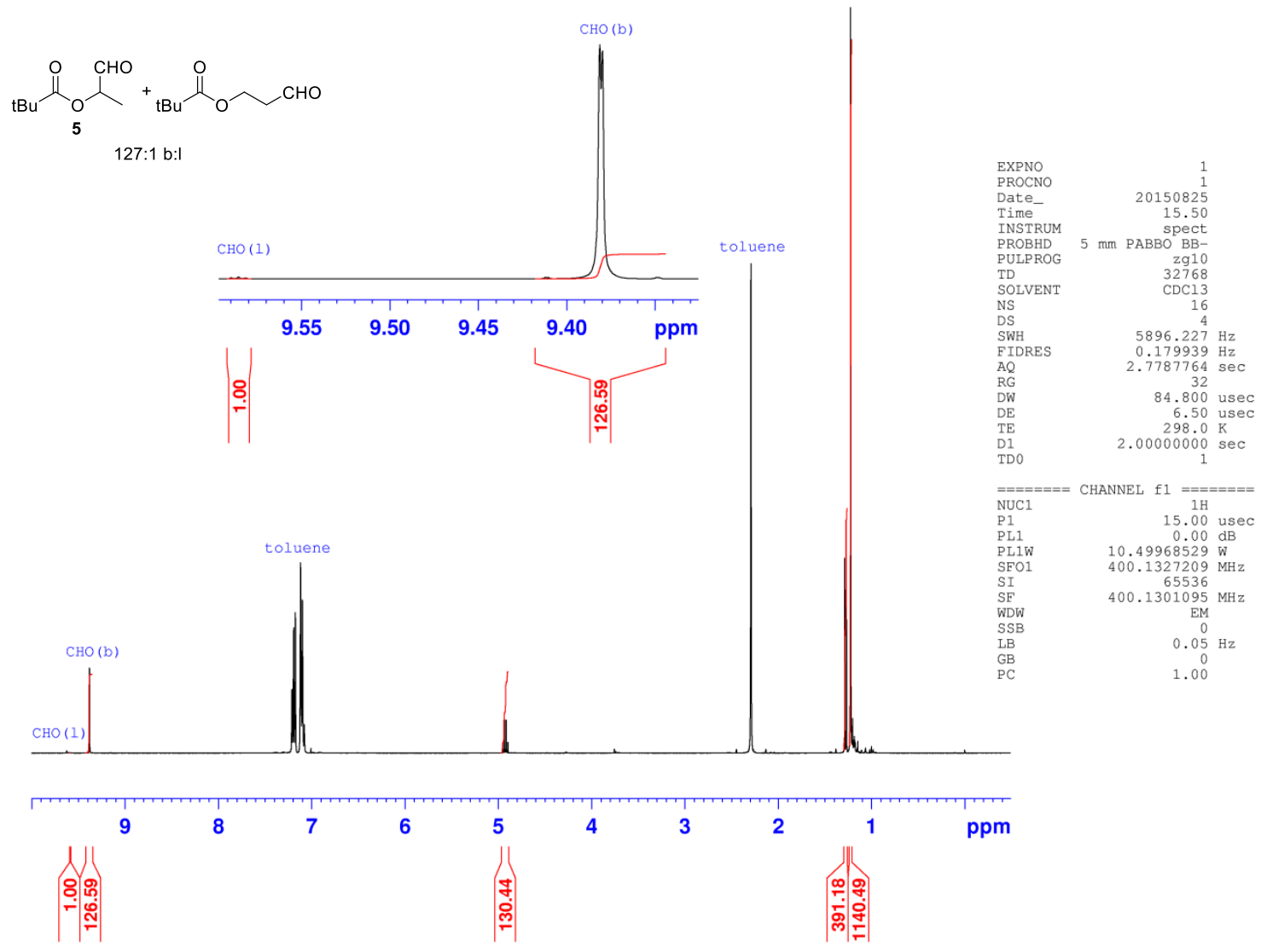




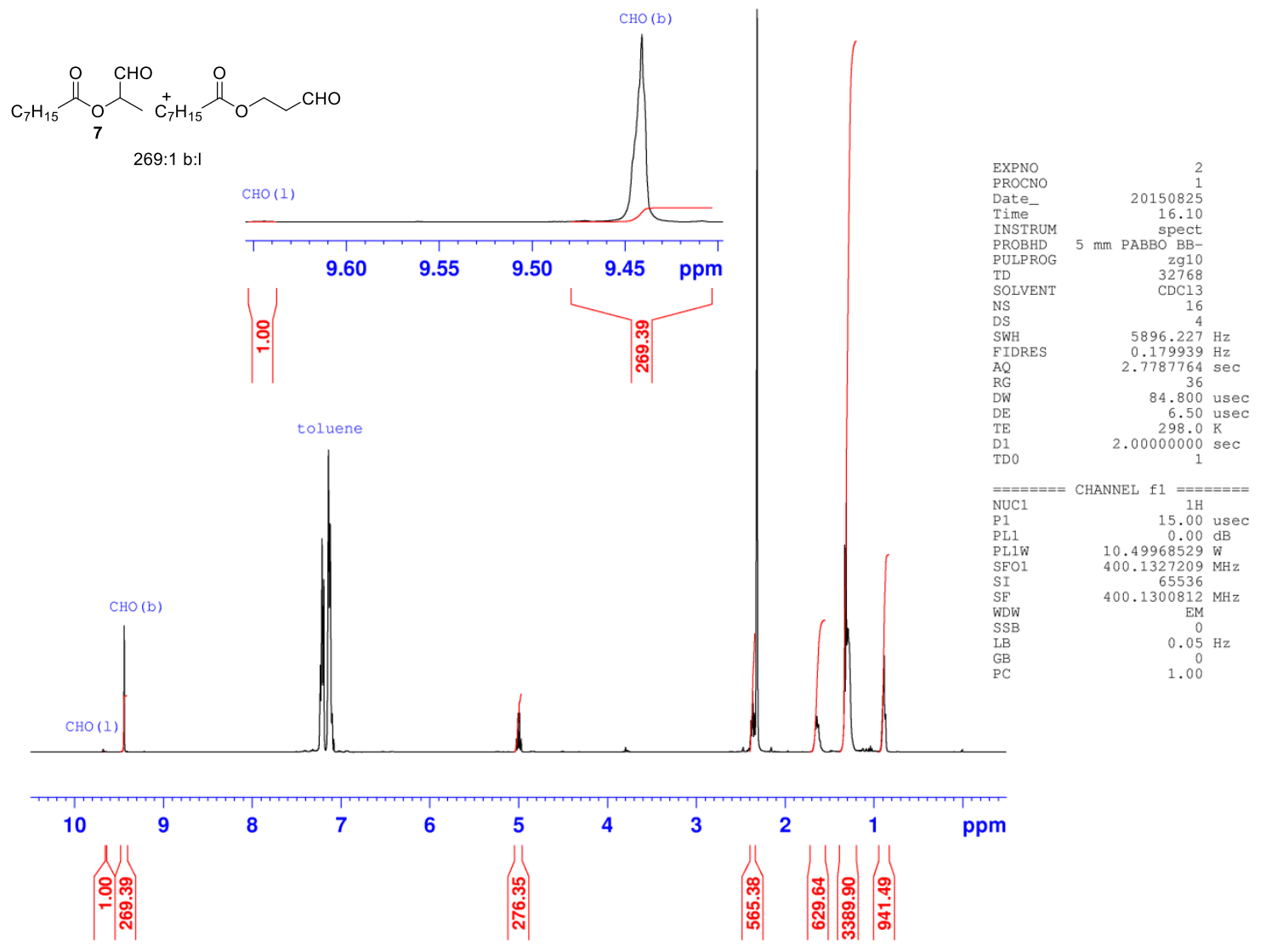




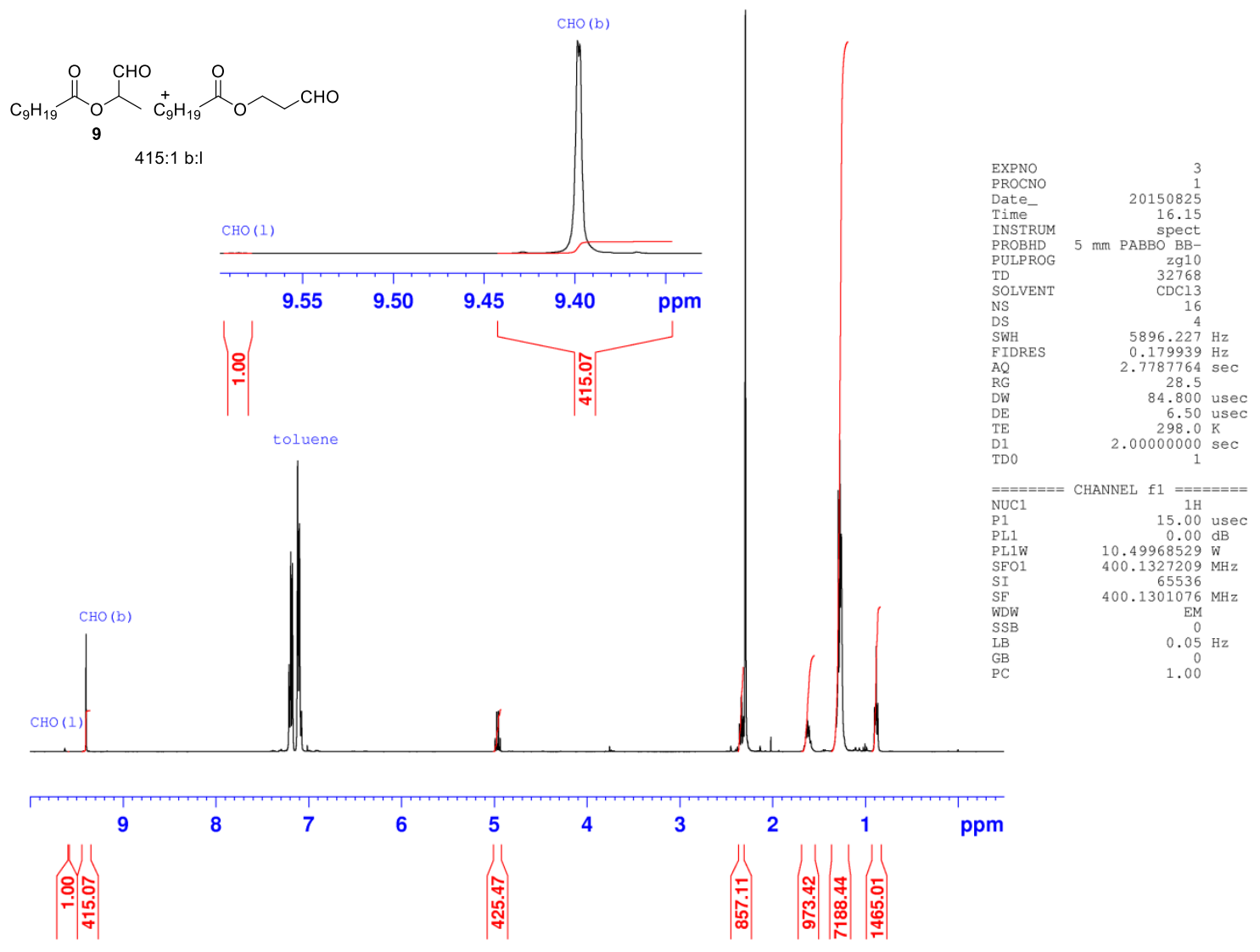




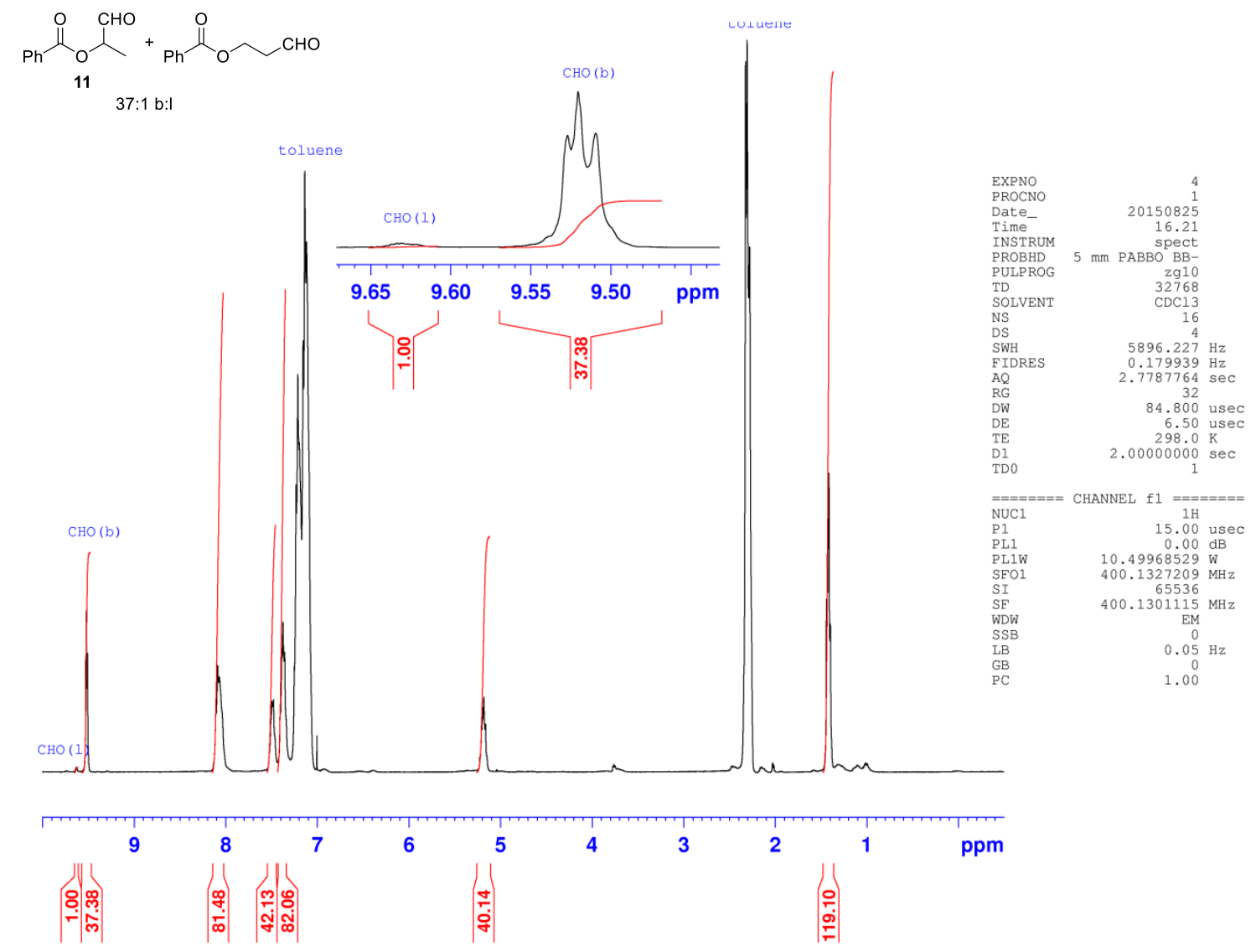




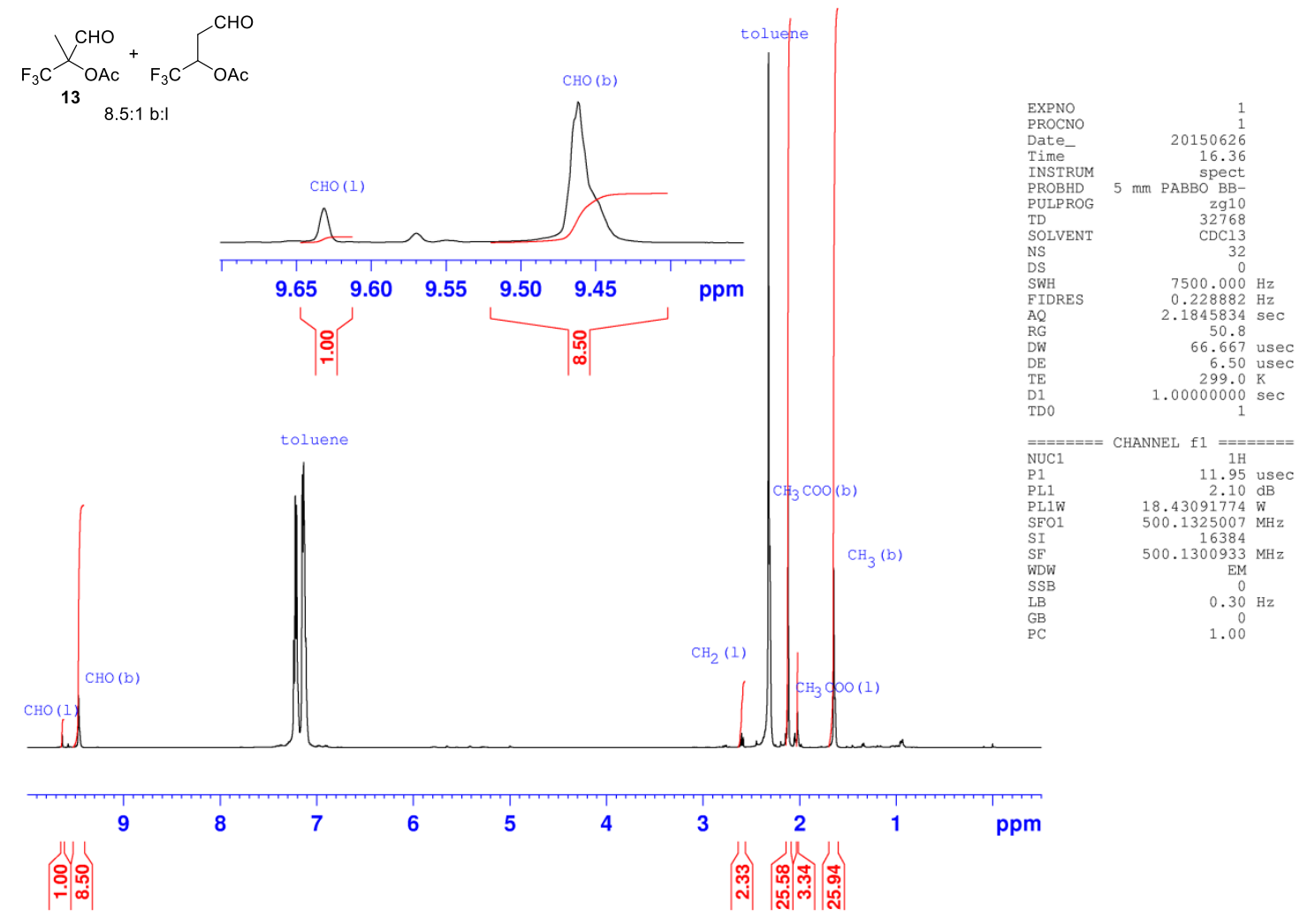




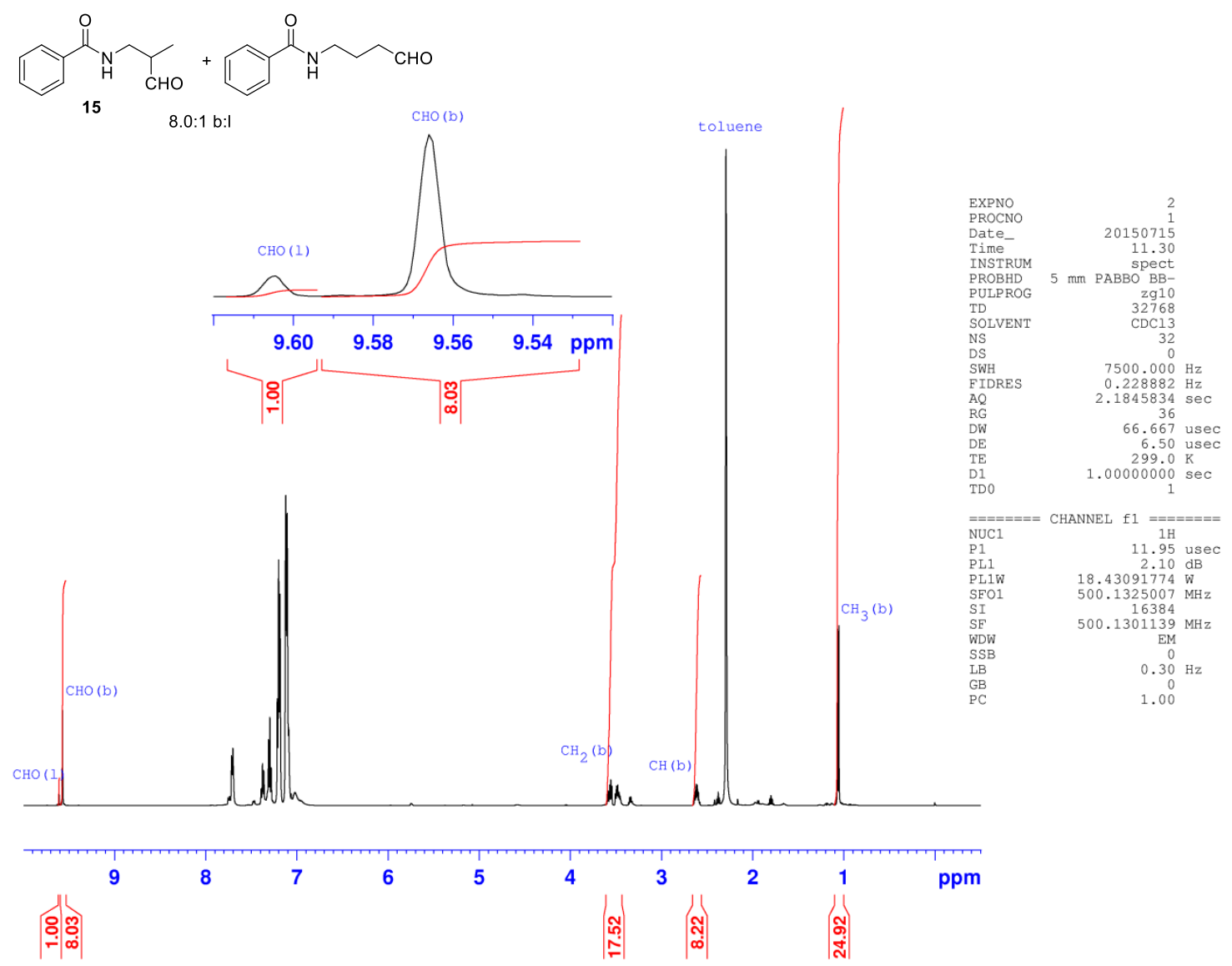




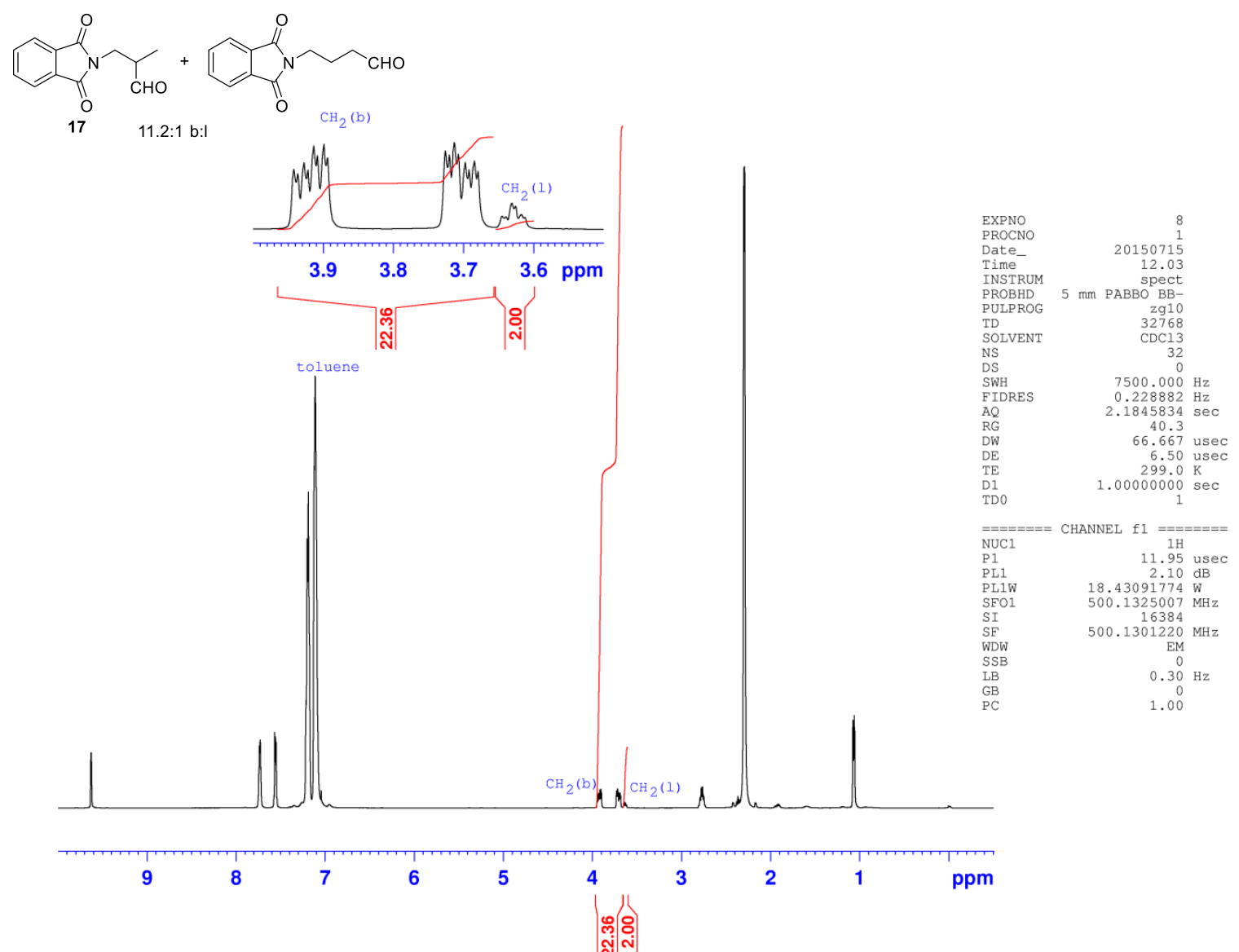

สี่ 


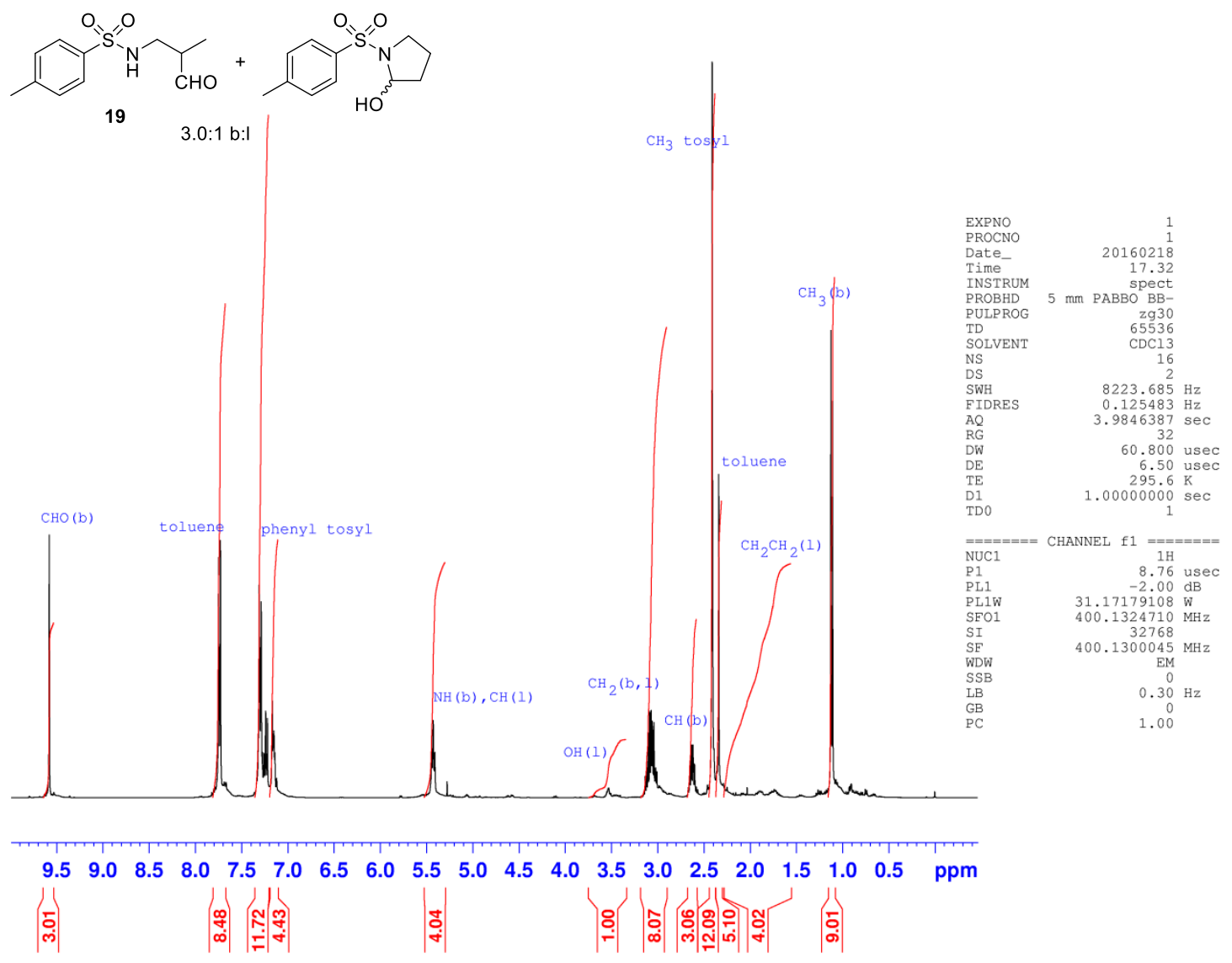



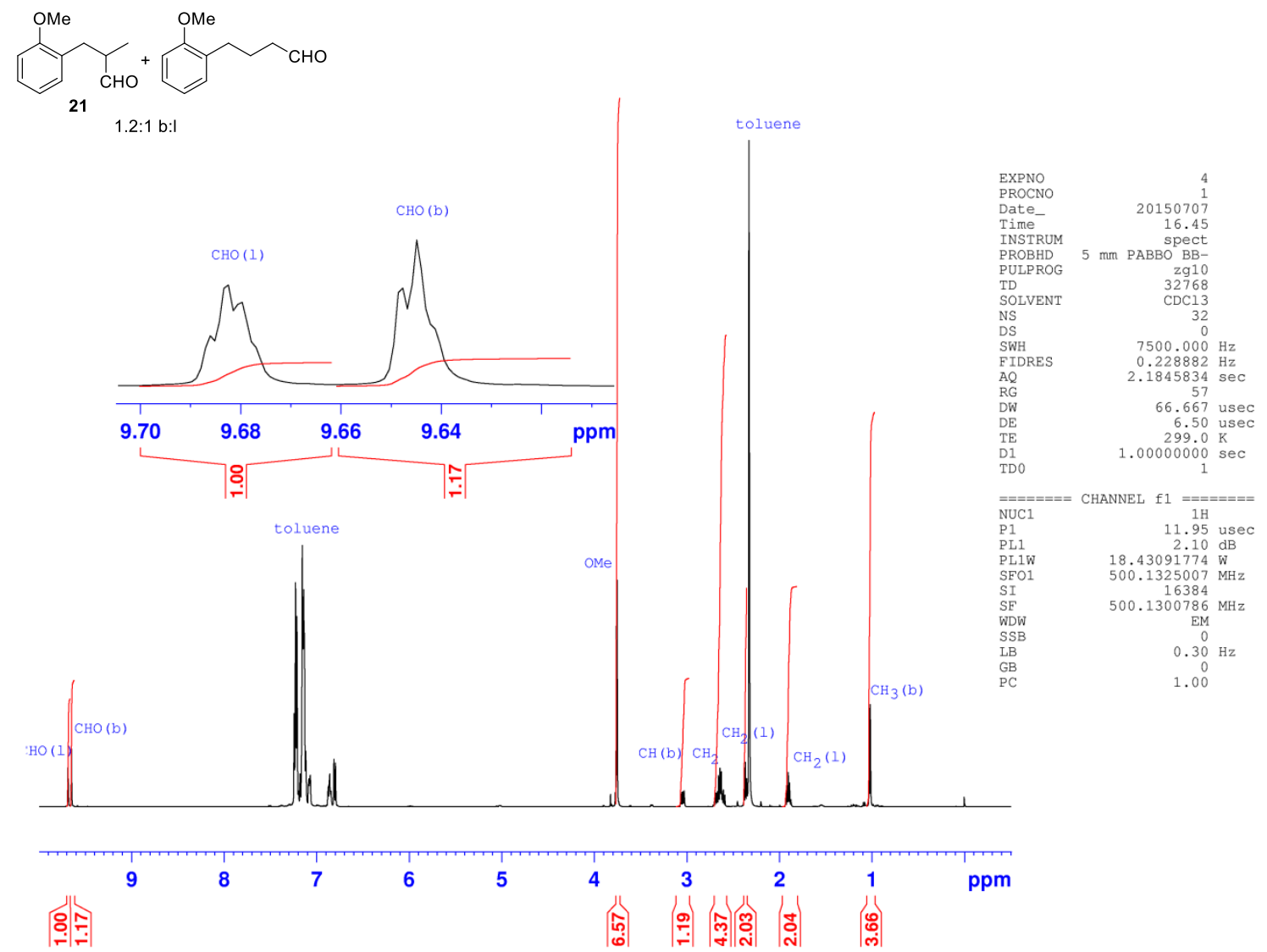

INSOBHD 5 spect 


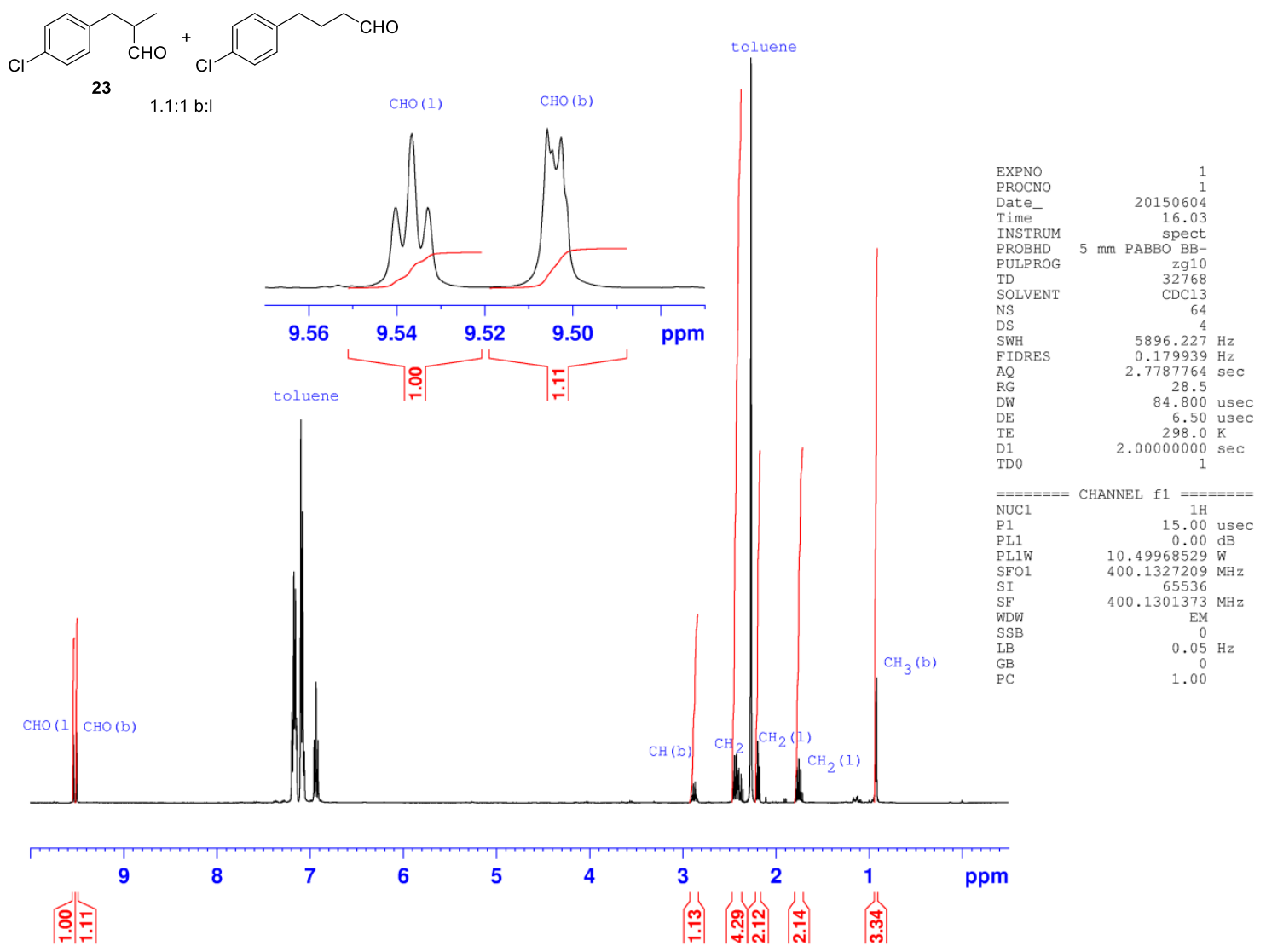



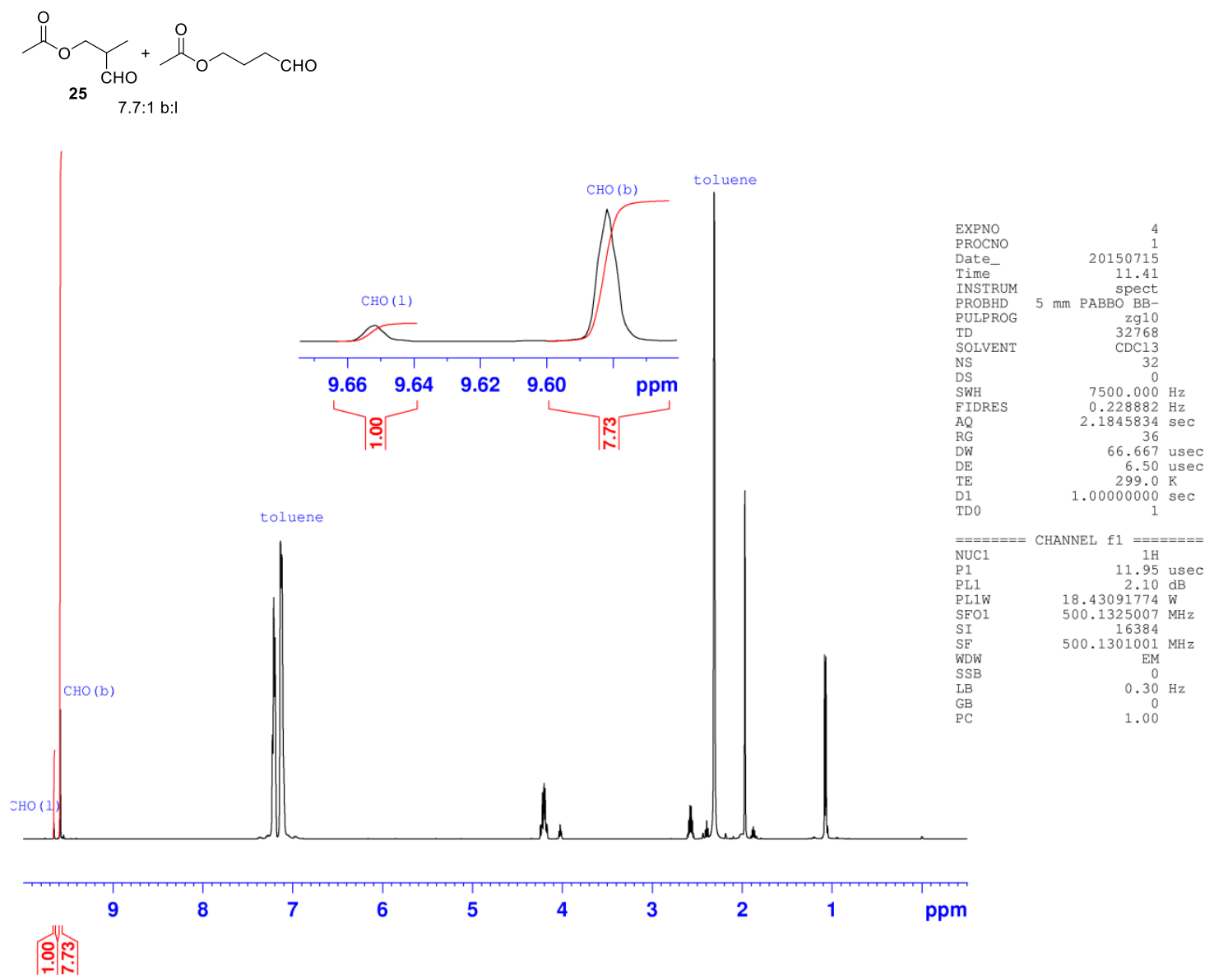


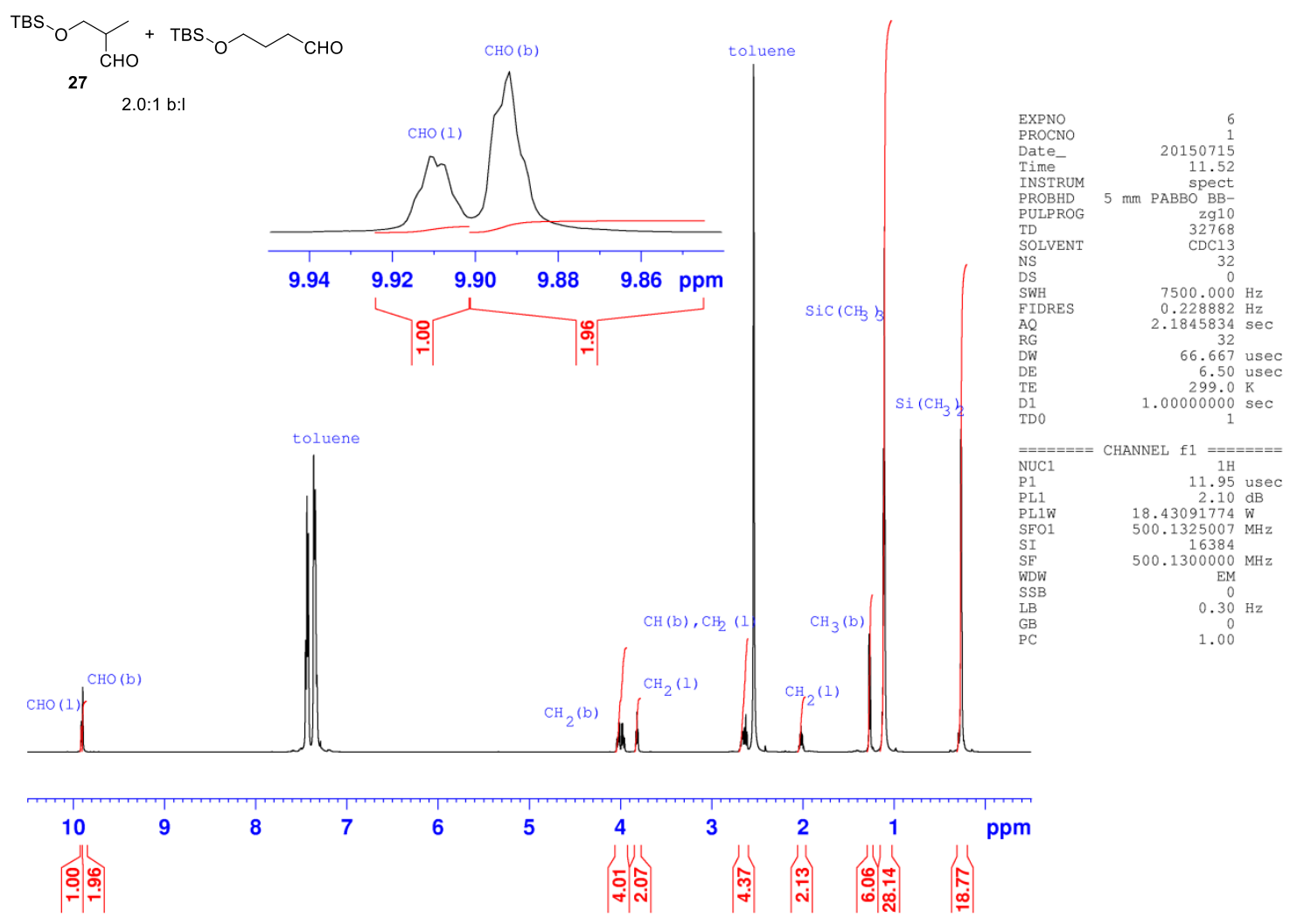




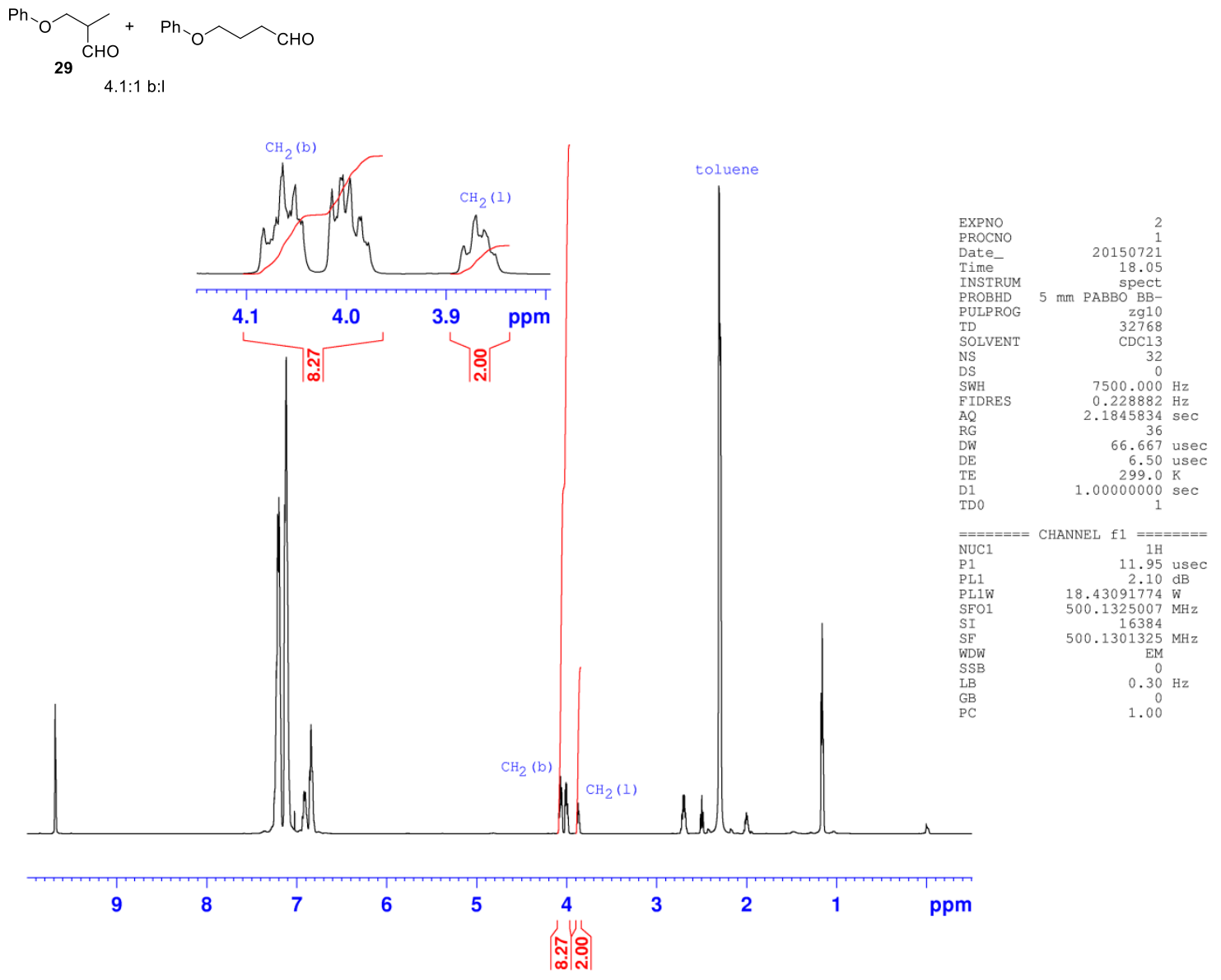




\section{Transition State Calculations}

Transition state searches were performed with the Gaussian 09 program $^{7}$ on a Unix HPC platform. All structures were optimized at the $\mathrm{UB}^{2} \mathrm{LYP}^{8}$ level of theory employing the LanL2DZ basis set with $\mathrm{ECP}$ for $\mathrm{Rh}^{9}$ and UB3LYP-gCP-D3/6-31G(d) for all other atoms with very tight optimization criteria. The modeling was based on the reported mechanism ${ }^{10}$ and all calculations were optimized in gas-phase. All optimized transition state structures were subjected to a frequency test to verify a single imaginary frequency was found (transition state) and that the imaginary vibrational mode corresponds with the expected reaction pathway. All stable structures were subjected to a frequency test to verify no imaginary frequencies. The resulting transition state structures were subjected to an IRC calculation to verify a clean reaction pathway from the starting geometry to the product geometry. The geometrical counterpoise (gCP) $)^{11}$ and dispersion (D3) ${ }^{12}$ corrections of Grimme et al. were applied to all stationary points to correct for basis set superposition error with 6-31G(d) and missing VDW dispersion in B3LYP, respectively. In comparison to results obtained with def2-QZVP, a much larger basis set than 6-31G(d), it has been demonstrated that the performance of B3LYP-gCP-D3/6-31G(d) is superior to B3LYP/6-31G(d) alone with respect to noncovalent interactions, reaction energies, and barrier heights. ${ }^{13}$ These corrections were applied for free at http://wwwtc.thch.uni-bonn.de/. Frequency corrections at $60^{\circ} \mathrm{C}$ were obtained using the freqchk utility included in the Gaussian09 suite of programs.

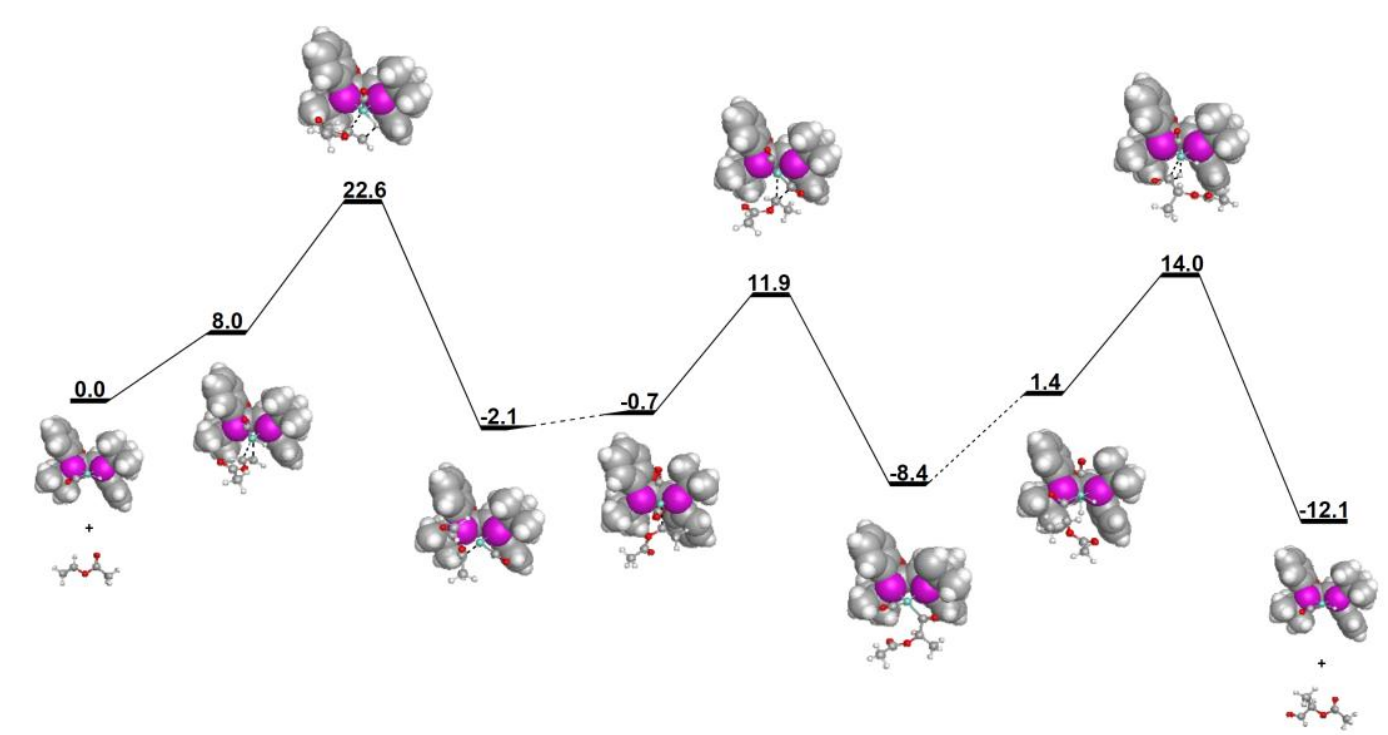

Figure S 1. Stationary points, in $\mathrm{kcal} / \mathrm{mol}$, along the free energy surface of the $\mathrm{Rh}$-catalyzed hydroformylation of vinylacetate with frequency corrections at $298 \mathrm{~K}$. The free energies of each elementary step are standardized by adding the free energies of $\mathrm{CO}$ and $\mathrm{H}_{2}$, where appropriate. All stationary points were computed in the gas phase. 


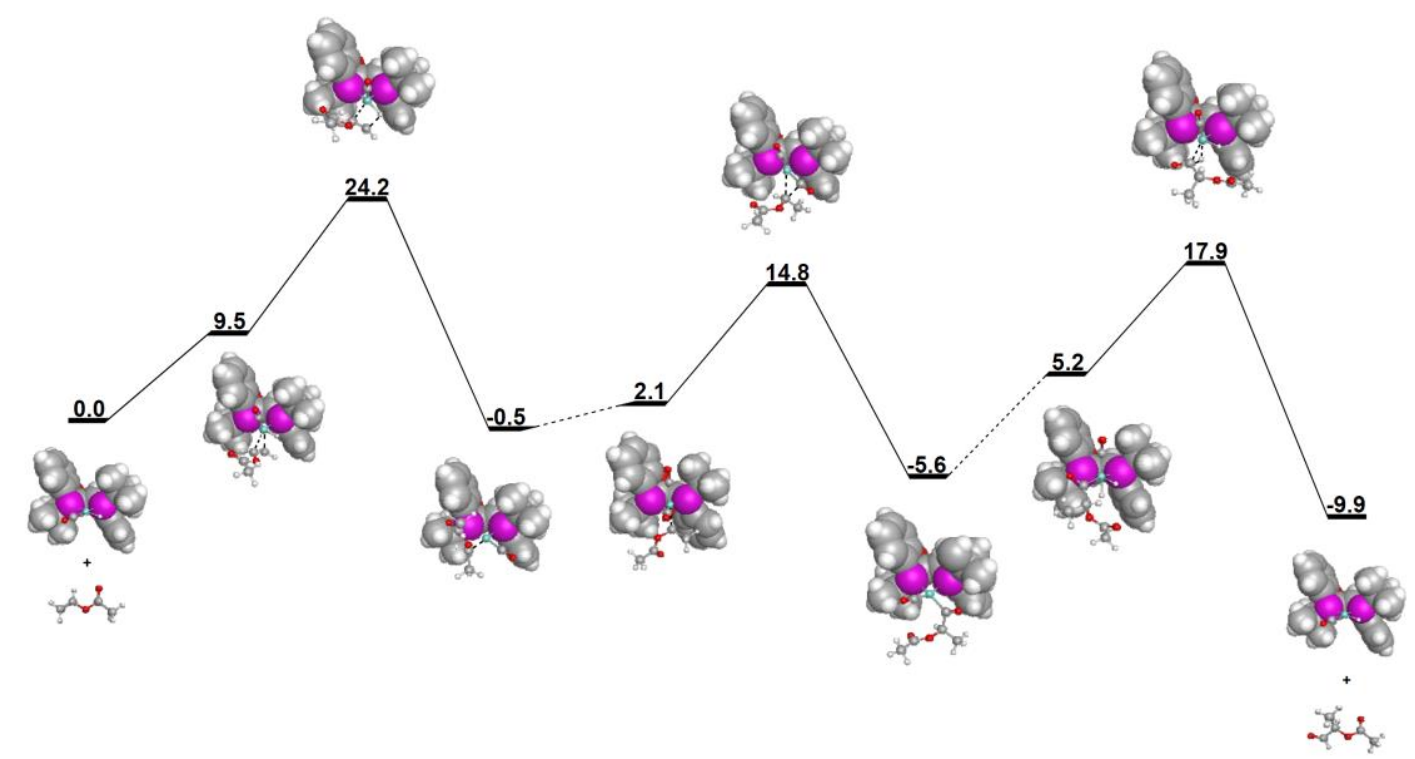

Figure S2. Stationary points, in $\mathrm{kcal} / \mathrm{mol}$, along the free energy surface of the $\mathrm{Rh}$-catalyzed hydroformylation of vinylacetate with frequency corrections at $333 \mathrm{~K}$. The free energies of each elementary step are standardized by adding the free energies of $\mathrm{CO}$ and $\mathrm{H}_{2}$, where appropriate. All stationary points were computed in the gas phase.

$\underline{\text { TS-A-1-SM }}$

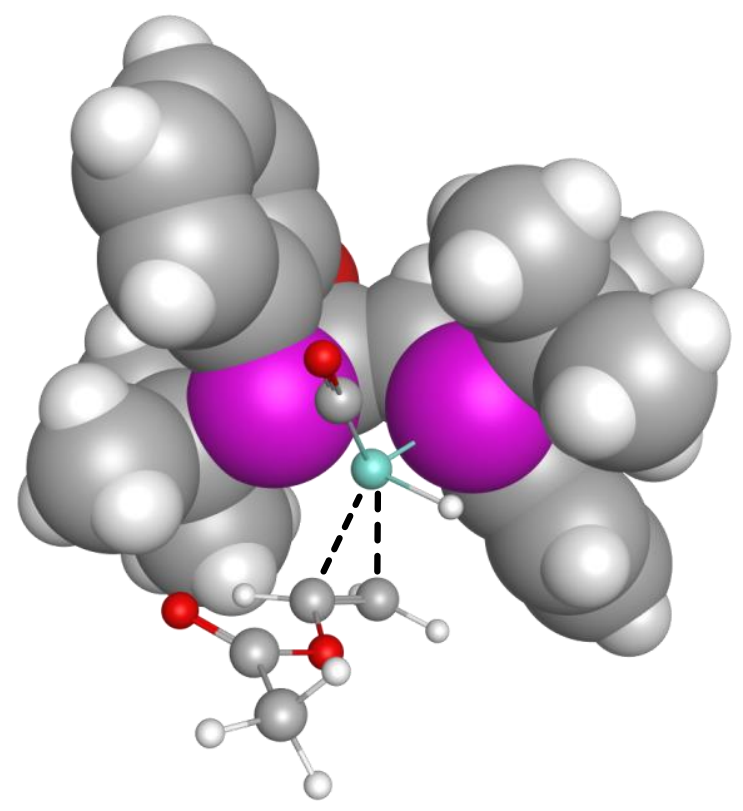

$\begin{array}{lrrr}\mathrm{P} & 2.472638872691 & -1.801090439517 & 0.787662693765 \\ \mathrm{P} & -0.224288095307 & -0.750194287102 & -0.665080220813 \\ \mathrm{C} & 2.540848559547 & -0.066615798825 & -0.001500328952 \\ \mathrm{H} & 2.613459929434 & 0.690075623518 & 0.783924973340 \\ \mathrm{C} & 1.333775922551 & 0.324624515107 & -0.888649959954 \\ \mathrm{H} & 1.666778237626 & 0.307594752356 & -1.929341338675 \\ \mathrm{C} & -1.142818235734 & -0.603908824924 & -2.340304768198 \\ \mathrm{C} & -2.583479358084 & -1.103007925420 & -2.098837220567\end{array}$




\begin{tabular}{|c|c|c|c|}
\hline $\mathrm{H}$ & -3.123656792781 & -1.128819224729 & -3.054151402019 \\
\hline H & -2.620212168373 & -2.107752667958 & -1.664479994635 \\
\hline H & -3.130477322212 & -0.428401572540 & -1.431176925724 \\
\hline C & -0.434048075320 & -1.509370373646 & -3.365146772854 \\
\hline H & -0.937259594456 & -1.422075922265 & -4.33673043244 \\
\hline H & 0.614416869632 & -1.224191491498 & -3.51654641785 \\
\hline $\mathrm{H}$ & -0.462245954771 & -2.561742270941 & -3.07337533259 \\
\hline C & -1.189531386830 & 0.837844106903 & -2.87960387922 \\
\hline H & -1.753862075103 & 0.847700476948 & -3.82153933521 \\
\hline H & -1.691442996870 & 1.518338925040 & -2.18569653931 \\
\hline H & -0.192967555816 & 1.240311610784 & -3.08885212492 \\
\hline C & 3.238909176086 & -1.535255446147 & 2.52033599784 \\
\hline C & 4.573755858261 & -0.770246827596 & 2.47190080759 \\
\hline H & 4.465044216510 & 0.240840557098 & 2.06414225733 \\
\hline $\mathrm{H}$ & 4.970725970322 & -0.669340834153 & 3.49058674717 \\
\hline H & 5.322408147437 & -1.298070235159 & 1.87307320979 \\
\hline C & 3.469638925650 & -2.934324236430 & 3.12447983800 \\
\hline $\mathrm{H}$ & 3.860322042195 & -2.832459016772 & 4.14534661412 \\
\hline H & 2.541029513803 & -3.512344567946 & 3.17085798222 \\
\hline $\mathrm{H}$ & 4.204082901829 & -3.507111582780 & 2.54651648408 \\
\hline C & 2.215569395530 & -0.765891951743 & $3.3757344572 \mathrm{~s}$ \\
\hline H & 2.012443141323 & 0.236500322528 & 2.97933107121 \\
\hline H & 1.263264827823 & -1.299789547260 & 3.44470165758 \\
\hline H & 2.610336278596 & -0.640397830636 & 4.39223266881 \\
\hline C & 3.885520423775 & -2.280490256262 & -0.26002276425 \\
\hline C & 4.369518922569 & -1.175649623788 & -0.97450216773 \\
\hline C & 4.482172909225 & -3.531357688906 & -0.44819086361 \\
\hline C & 5.451823274379 & -1.287221489547 & -1.8480391286 \\
\hline C & 5.567592659483 & -3.659911327977 & -1.31740881893 \\
\hline C & 6.046526854739 & -2.539762406300 & $-2.0073456695 \mathrm{~s}$ \\
\hline H & 5.807781622017 & -0.413126965785 & -2.38388888215 \\
\hline H & 6.035720892031 & -4.629082246845 & $-1.4618725660 \mathrm{~s}$ \\
\hline H & 6.888682787777 & -2.643853303573 & -2.68616488301 \\
\hline C & -0.942322790342 & 0.614035789254 & 0.31355817510 \\
\hline C & -0.158059617522 & 1.769678840765 & 0.1849359644 \\
\hline C & -2.095489273270 & 0.651530061335 & 1.10284534615 \\
\hline C & -0.510698059460 & 2.965476498259 & 0.81011365475 \\
\hline C & -2.461461366563 & 1.841475648996 & 1.73656199123 \\
\hline C & -1.672711412321 & 2.987581698941 & 1.58361708375 \\
\hline $\mathrm{H}$ & 0.115072777793 & 3.844302291358 & 0.69222358760 \\
\hline H & -3.355332094487 & 1.874182430392 & 2.35204942028 \\
\hline $\mathrm{H}$ & -1.959507838072 & 3.909762829581 & 2.08180792506 \\
\hline 0 & 3.753113120293 & 0.029709194358 & -0.78430431242 \\
\hline 0 & 0.964250543440 & 1.692013877015 & -0.59496420024 \\
\hline H & -2.698759553733 & -0.242571757557 & 1.22971142989 \\
\hline $\mathrm{H}$ & 4.096935183819 & -4.399610192187 & 0.07919969018 \\
\hline $\mathrm{Rh}$ & 0.339415647473 & -2.821623328811 & 0.51021910487 \\
\hline C & 0.835209947798 & -4.218930174247 & -1.16080873826 \\
\hline C & -0.528753201749 & -4.330053546805 & -0.85764535117 \\
\hline $\mathrm{H}$ & -1.301971842548 & -3.867939144540 & -1.45674688687 \\
\hline 0 & -0.973386042488 & -5.508020865905 & -0.22967300762 \\
\hline C & -2.292272740288 & -5.575648984685 & 0.08170038323 \\
\hline 0 & -3.098686919249 & -4.713027036573 & -0.19290377986 \\
\hline C & -2.597454929244 & -6.851418043208 & 0.82917437252 \\
\hline
\end{tabular}




$\begin{array}{lrrr}\mathrm{H} & -2.086901439462 & -7.704521444156 & 0.373629633644 \\ \mathrm{H} & -2.234172087887 & -6.759920285164 & 1.859729503836 \\ \mathrm{H} & -3.675964851316 & -7.014986554589 & 0.844719784707 \\ \mathrm{H} & 0.851201538554 & -4.052021397911 & 1.372950859936 \\ \mathrm{C} & -0.889743140690 & -2.566985112423 & 1.971360921462 \\ \mathrm{O} & -1.586922411362 & -2.514934799648 & 2.892922914012 \\ \mathrm{H} & 1.512941062445 & -5.001525395014 & -0.837167717738 \\ \mathrm{H} & 1.145125369254 & -3.667976602143 & -2.041073474599\end{array}$

SCF Done: $E$ (UB3LYP) $=-2218.316581$

DFT-gCP-D3 E (UB3LYP) $=-2218.351715$

gCP correction=

0.163910

D3 correction=

$-0.199044$

Zero-point correction=

0.567970

Thermal correction to Energy=

0.606060

Thermal correction to Enthalpy=

Thermal correction to Gibbs Free Energy=

Sum of electronic and zero-point Energies=

Sum of electronic and thermal Energies=

Sum of electronic and thermal Enthalpies=

0.607004

0.497049

$-2217.783745$

$-2217.745655$

$-2217.744711$

Sum of electronic and thermal Free Energies=

$-2217.854666$

TS-A-1

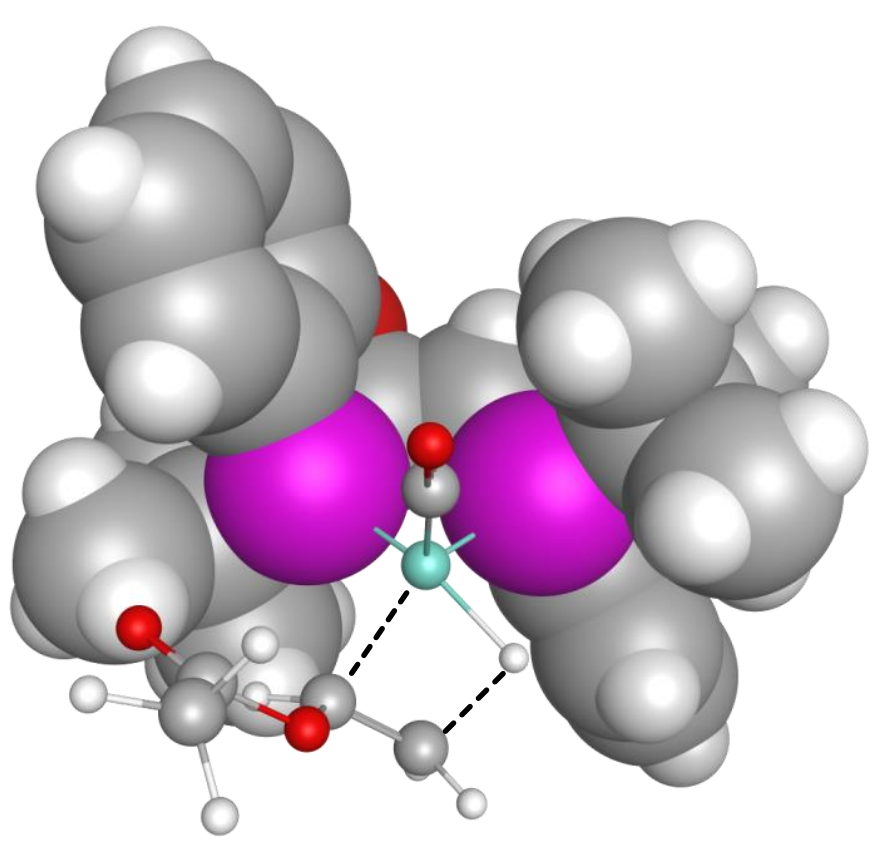

$\begin{array}{lrrr}\text { P } & 0.767240351592 & -1.306218440179 & 0.247957680699 \\ \text { P } & -1.832605890323 & -0.050730418039 & -1.129851536596 \\ \mathrm{C} & 0.784679888995 & 0.579760668827 & 0.032665816249 \\ \mathrm{H} & 0.737658133377 & 1.075324642281 & 1.005228628393 \\ \mathrm{C} & -0.365622022958 & 1.135675031848 & -0.832655951551 \\ \mathrm{H} & 0.070705553871 & 1.427768443947 & -1.790710070303 \\ \mathrm{C} & -2.423577661627 & 0.456734238231 & -2.887776381811 \\ \mathrm{C} & -3.899261447879 & 0.030355251846 & -3.031560403287\end{array}$




\begin{tabular}{|c|c|c|c|}
\hline $\mathrm{H}$ & -4.214032710900 & 0.164525212023 & -4.075114615478 \\
\hline $\mathrm{H}$ & -4.085483503914 & -1.009405741120 & -2.749750688566 \\
\hline H & -4.548976967605 & 0.654098803475 & -2.408738316445 \\
\hline C & -1.542402425997 & -0.296128016500 & -3.901906497696 \\
\hline H & -1.843547549672 & -0.030235158551 & -4.923630946697 \\
\hline $\mathrm{H}$ & -0.480954177787 & -0.040986361721 & -3.794045629985 \\
\hline H & -1.633457348620 & -1.381267178448 & -3.796092162336 \\
\hline C & -2.330468240703 & 1.972443682218 & -3.148249267567 \\
\hline H & -2.747728868251 & 2.188444406577 & -4.140964917432 \\
\hline H & -2.905744945509 & 2.547869201245 & -2.416642767594 \\
\hline $\mathrm{H}$ & -1.300664033079 & 2.344031018032 & -3.142628037297 \\
\hline C & 1.483829359614 & -1.570291949933 & 2.003636319588 \\
\hline C & 2.807402318381 & -0.814031864881 & 2.219208848565 \\
\hline $\mathrm{H}$ & 2.694464353341 & 0.268508236483 & 2.096976697514 \\
\hline $\mathrm{H}$ & 3.163861439599 & -0.994323850542 & 3.241864007876 \\
\hline H & 3.587433360686 & -1.152377304902 & 1.530542391582 \\
\hline C & 1.720791130948 & -3.084276403965 & 2.166313333017 \\
\hline H & 2.093660137724 & -3.291633192360 & 3.177803739523 \\
\hline $\mathrm{H}$ & 0.797788716139 & -3.654870028879 & 2.019938236269 \\
\hline $\mathrm{H}$ & 2.470861621307 & -3.453194405669 & 1.456844127366 \\
\hline C & 0.438514456120 & -1.095660160079 & 3.029679280268 \\
\hline $\mathrm{H}$ & 0.258763834182 & -0.015661736181 & 2.967883405913 \\
\hline $\mathrm{H}$ & -0.520041599976 & -1.605753682712 & 2.902548686199 \\
\hline $\mathrm{H}$ & 0.803060850800 & -1.306471312312 & 4.043323470693 \\
\hline C & 2.229788039695 & -1.399006699872 & -0.839916574626 \\
\hline C & 2.710020667094 & -0.111023670041 & -1.118853264902 \\
\hline C & 2.868174160834 & -2.505533501584 & -1.407352136054 \\
\hline C & 3.826964277069 & 0.091694258504 & -1.929972523446 \\
\hline C & 3.988337088197 & -2.319898044505 & -2.220767788488 \\
\hline C & 4.461429841646 & -1.026595138077 & -2.473393481557 \\
\hline H & 4.177677559698 & 1.099724556199 & -2.126995453637 \\
\hline H & 4.488547102641 & -3.177622623677 & -2.660728099955 \\
\hline $\mathrm{H}$ & 5.330405158255 & -0.885233487455 & -3.110427452162 \\
\hline C & -2.836383268385 & 1.012560924958 & -0.039419259644 \\
\hline C & -2.138805161039 & 2.180993860330 & 0.293065437575 \\
\hline C & -4.115028290238 & 0.798666476726 & 0.483109181005 \\
\hline C & -2.702089631590 & 3.157501548122 & 1.114981086165 \\
\hline C & -4.692266070452 & 1.766607733301 & 1.308418815138 \\
\hline C & -3.986955534048 & 2.936777869632 & 1.614770576775 \\
\hline H & -2.141098715264 & 4.054997319411 & 1.355494995383 \\
\hline H & -5.686562284259 & 1.609007017736 & 1.715699539115 \\
\hline H & -4.438784941307 & 3.684987125262 & 2.260661638598 \\
\hline 0 & 2.052976813211 & 0.950369926297 & -0.560263941295 \\
\hline 0 & -0.881095940920 & 2.341964009041 & -0.224218476140 \\
\hline H & -4.648798042632 & -0.117701822341 & 0.244930176098 \\
\hline H & 2.488112357315 & -3.506203857960 & -1.218709992965 \\
\hline $\mathrm{Rh}$ & -1.280527236821 & -2.272934869566 & -0.385021371322 \\
\hline C & -1.215653491652 & -3.971243767420 & -1.956516160018 \\
\hline C & -2.512709414329 & -3.418078753862 & -1.758866322912 \\
\hline H & -2.946969924817 & -2.744960790397 & -2.485693046795 \\
\hline 0 & -3.458727485452 & -4.306615026270 & -1.196936168275 \\
\hline C & -4.714859029504 & -3.840590184887 & -0.994862802558 \\
\hline 0 & -5.092710231597 & -2.740047369551 & -1.338691121384 \\
\hline C & -5.552681531180 & -4.861461064943 & -0.264590031941 \\
\hline
\end{tabular}




$\begin{array}{ll}\mathrm{H} & -5.379507724706 \\ \mathrm{H} & -5.264748589816 \\ \mathrm{H} & -6.607223365952 \\ \mathrm{H} & -0.502891310466 \\ \mathrm{C} & -2.342203154038 \\ \mathrm{O} & -3.019709376932 \\ \mathrm{H} & -1.075173866513 \\ \mathrm{H} & -0.615228963621\end{array}$

$-5.866925455732$

$-4.863078989628$

$-4.594542760895$

$-3.709283135637$

$-2.649611744640$

$-2.955903434396$

$-5.019546832753$

$-3.622841629489$
$-0.657963677747$

0.793378179158

$-0.347482790650$

$-0.487476660839$

1.221470680360

2.108635598191

$-1.703389024331$

$-2.789411758990$

SCF Done: $E$ (UB3LYP) $=-2218.29286699$

gCP correction=

D3 correction=

DFT-gCP-D3 E (UB3LYP) $=-2218.32615082$

Zero-point correction=

Thermal correction to Energy=

Thermal correction to Enthalpy=

Thermal correction to Gibbs Free Energy=

Sum of DFT-gCP-D3 and zero-point Energies=

Sum of DFT-gCP-D3 and thermal Energies=

Sum of DFT-gCP-D3 and thermal Enthalpies=

Sum of DFT-gCP-D3 and thermal Free Energies=

0.565527

0.165444

$-0.198728$

0.603692

0.604636

0.494865

$-2217.760624$

$-2217.722459$

$-2217.721515$

$-2217.831286$

TS-A-1-PDT

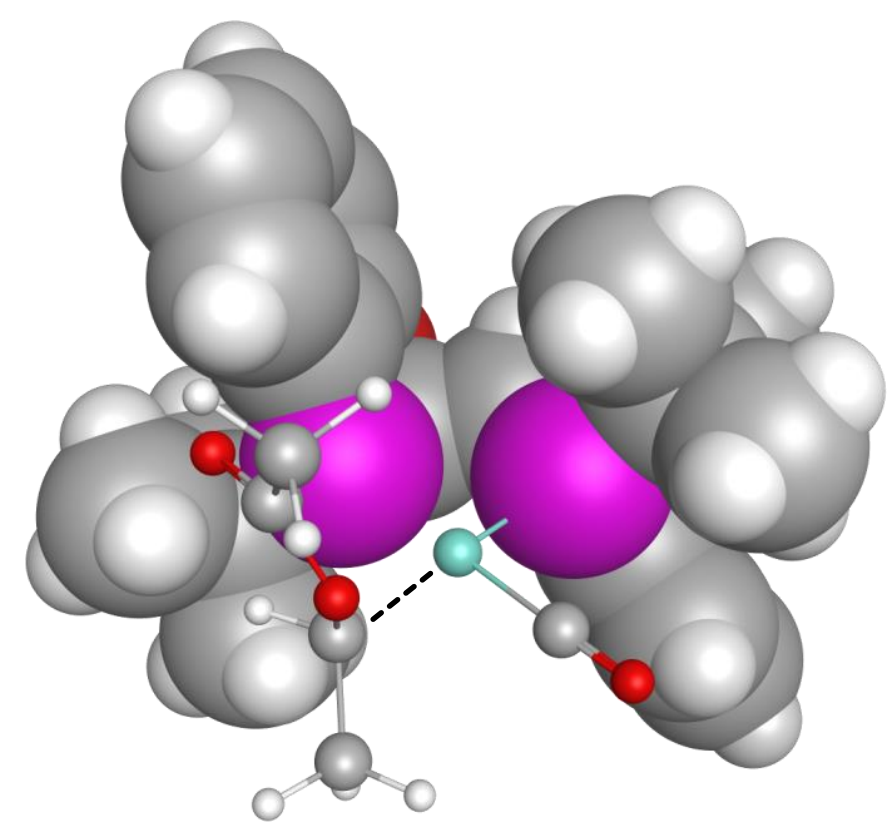

$\begin{array}{lr}\mathrm{P} & 1.040417018100 \\ \mathrm{P} & -1.523835448209 \\ \mathrm{C} & 0.978003440470 \\ \mathrm{H} & 0.729529449396 \\ \mathrm{C} & -0.046087025929 \\ \mathrm{H} & 0.479434205935 \\ \mathrm{C} & -2.219307425305 \\ \mathrm{C} & -3.735727282422\end{array}$

$-1.836082135558$

$-0.623218828981$

0.045416611077

0.514110595146

0.547984327187

0.743732669452

$-0.117612847278$

$-0.407818847388$
0.183680368020

$-1.320211479369$

$-0.012260333160$

0.943140720471

$-1.051211512629$

$-1.988206981748$

$-3.034023037760$

$-3.036243549499$ 


\begin{tabular}{|c|c|}
\hline $\mathrm{H}$ & -4.120776127236 \\
\hline $\mathrm{H}$ & -3.987828351068 \\
\hline $\mathrm{H}$ & -4.270303267926 \\
\hline C & -1.495701374789 \\
\hline H & -1.881853917893 \\
\hline H & -0.415382119214 \\
\hline $\mathrm{H}$ & -1.643071135061 \\
\hline C & -2.014718110851 \\
\hline $\mathrm{H}$ & -2.494530415281 \\
\hline H & -2.471926091250 \\
\hline $\mathrm{H}$ & -0.958972264116 \\
\hline C & 1.413974762549 \\
\hline C & 2.517515742738 \\
\hline $\mathrm{H}$ & 2.249184887221 \\
\hline $\mathrm{H}$ & 2.693685141466 \\
\hline $\mathrm{H}$ & 3.463189053618 \\
\hline C & 1.861887100494 \\
\hline $\mathrm{H}$ & 2.057973798978 \\
\hline $\mathrm{H}$ & 1.096378783990 \\
\hline $\mathrm{H}$ & 2.787018999653 \\
\hline C & 0.095858267331 \\
\hline $\mathrm{H}$ & -0.257803088076 \\
\hline $\mathrm{H}$ & -0.698189325868 \\
\hline $\mathrm{H}$ & 0.247084575054 \\
\hline C & 2.670954993750 \\
\hline C & 3.113089872326 \\
\hline C & 3.467825689659 \\
\hline C & 4.346541160096 \\
\hline C & 4.704761296164 \\
\hline C & 5.136346653043 \\
\hline $\mathrm{H}$ & 4.665521869268 \\
\hline $\mathrm{H}$ & 5.327885077138 \\
\hline $\mathrm{H}$ & 6.097698364377 \\
\hline C & -2.449613464291 \\
\hline C & -1.770900115087 \\
\hline C & -3.670987369334 \\
\hline C & -2.293097640239 \\
\hline C & -4.200227715905 \\
\hline C & -3.516972746164 \\
\hline H & -1.747257330207 \\
\hline H & -5.147107261798 \\
\hline $\mathrm{H}$ & -3.936697300103 \\
\hline 0 & 2.299097863107 \\
\hline 0 & -0.578217060590 \\
\hline $\mathrm{H}$ & -4.194436234419 \\
\hline $\mathrm{H}$ & 3.121637397409 \\
\hline $\mathrm{Rh}$ & -0.805900439305 \\
\hline C & -2.109999102488 \\
\hline C & -2.452764974227 \\
\hline $\mathrm{H}$ & -3.095773922510 \\
\hline 0 & -3.277266242088 \\
\hline C & -4.256600558672 \\
\hline 0 & -4.574893689435 \\
\hline C & -4.916033468794 \\
\hline
\end{tabular}

$-0.280856930558$

$-1.415682002297$

0.294017015697

$-0.975893809315$

$-0.731716248291$

$-0.784878860737$

$-2.045545763589$

1. 374284510987

1. 591917995253

2. 024997260434

1. 646938515051

$-2.084069374257$

$-1.143995105901$

$-0.086405404821$

$-1.345531381294$

$-1.300398082873$

$-3.545257408898$

$-3.721942881439$

$-4.258320846568$

$-3.763808396047$

$-1.839826383313$

$-0.806981218795$

$-2.504672709223$

$-2.027904104775$

$-1.820664841696$

$-0.501030915985$

$-2.879076153007$

$-0.218416973567$

$-2.611784051936$

$-1.288234791947$

0.811615974669

$-3.429965606437$

$-1.084808948926$

0.421660367576

1.635510926458

0.197467379366

2. 639065970170

1. 195462733439

2. 404377629476

3. 567387358641

1. 030113890607

3. 173586170399

0.509169309227

1.810074470712

$-0.742414518719$

$-3.903483340666$

$-2.916378924124$

$-4.885838233954$

$-3.856852662673$

$-3.093197195113$

$-4.566196708086$

$-3.899066791618$

$-2.745805935430$

$-4.737153899723$
$-4.056579432562$

$-2.698588770169$

$-2.388382484589$

$-4.089074678654$

$-5.087298603835$

$-4.098957658904$

$-3.918593602761$

$-3.357191306557$

$-4.320592005972$

$-2.606402637847$

$-3.451624496971$

2. 040983230307

2. 557344080221

2. 466215525909

3. 622109548399

2. 028715274627

2. 239276101818

3. 304750524577

1. 920235628185

1. 694085500093

2.800336218189

2. 696788586487

2. 443119015498

3. 871243775295

$-0.627991150203$

$-0.804646190799$

$-1.075959136132$

$-1.392176980916$

$-1.665925493280$

$-1.815936375798$

$-1.514500934217$

$-2.014207161210$

$-2.279884612880$

$-0.139058622754$

0.058630261948

0.505233976707

0.873314394133

1. 328321120974

1.503300213712

1.007961229496

1.833835220762

2.146253805326

$-0.371243830057$

$-0.594238412401$

0.357666958365

$-0.969842274031$

$-0.836373879711$

$-2.902287639258$

$-1.831559377110$

$-2.262513643908$

$-0.844961761991$

$-0.236058453405$

$-0.485837979029$

0.838556839490 


$\begin{array}{lrrr}\mathrm{H} & -4.932473618358 & -5.794644996373 & 0.563785686486 \\ \mathrm{H} & -4.342088194571 & -4.640867230888 & 1.768593273903 \\ \mathrm{H} & -5.929088556300 & -4.371789706855 & 1.018225116370 \\ \mathrm{H} & -1.572254691356 & -5.746778411902 & -2.492880262624 \\ \mathrm{C} & -0.180861902648 & -4.646760644713 & -0.499730276043 \\ \mathrm{O} & 0.231624578231 & -5.707686404595 & -0.285362553067 \\ \mathrm{H} & -3.016860355143 & -5.263268123702 & -3.399221170071 \\ \mathrm{H} & -1.468846817038 & -4.429342896194 & -3.664915451884\end{array}$

SCF Done: $E$ (UB3LYP) $=-2218.34222017$

gCP correction=

D3 correction=

DFT-gCP-D3 E (UB3LYP) $=-2218.37157655$

Zero-point correction=

Thermal correction to Energy=

0.610540

Thermal correction to Enthalpy=

0.611484

Thermal correction to Gibbs Free Energy=

Sum of DFT-gCP-D3 and zero-point Energies=

0.500943

Sum of DFT-gCP-D3 and thermal Energies=

Sum of DFT-gCP-D3 and thermal Enthalpies=

$-2217.799309$

$-2217.761037$

$-2217.760093$

Sum of DFT-gCP-D3 and thermal Free Energies=

$-2217.870634$

$\underline{\text { TS-B-1-SM }}$

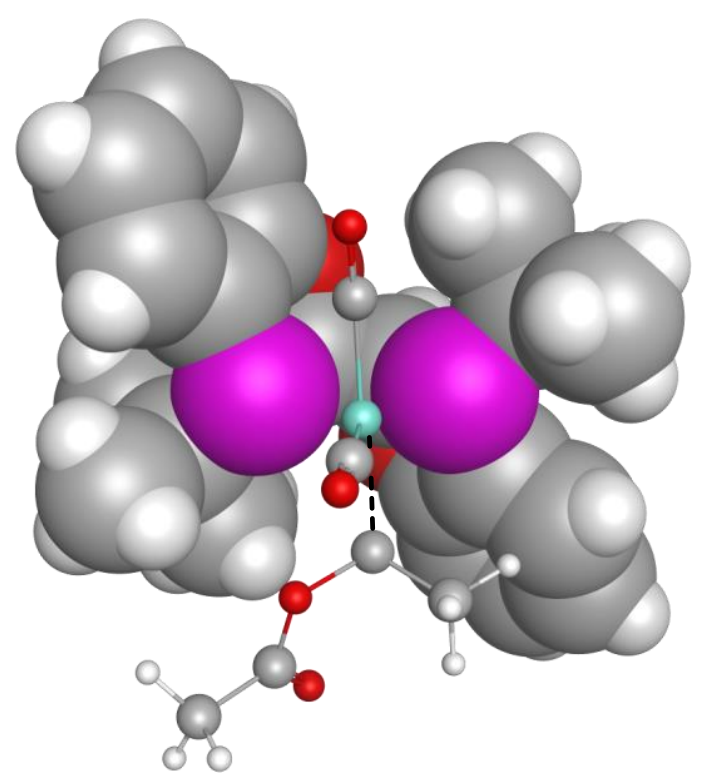

$\begin{array}{lllr}\text { P } & 4.084493129041 & 2.421311441678 & -0.957129660523 \\ \text { P } & 7.214276792784 & 2.851827262644 & 0.178867216172 \\ \text { C } & 4.458876656104 & 3.833563530779 & 0.289153682773 \\ \text { H } & 3.905110640801 & 4.736823597903 & 0.004868239017 \\ \text { C } & 5.923996368437 & 4.231527105782 & 0.474836499420 \\ \text { H } & 6.020786057466 & 4.626680491333 & 1.492947471953 \\ \text { C } & 8.100428446968 & 2.691411833558 & 1.868771378682 \\ \text { C } & 9.223945637407 & 1.655142008369 & 1.665737591292 \\ \text { H } & 9.708501095550 & 1.444586156898 & 2.628151410306\end{array}$




\begin{tabular}{|c|c|}
\hline H & 8.833286058768 \\
\hline H & 9.998042284899 \\
\hline C & 7.081510376355 \\
\hline H & 7.583904505593 \\
\hline H & 6.249413407807 \\
\hline $\mathrm{H}$ & 6.660605146669 \\
\hline C & 8.703137167204 \\
\hline H & 9.222509094494 \\
\hline H & 9.427532911205 \\
\hline H & 7.938390345175 \\
\hline C & 3.043086835676 \\
\hline C & 1.764630528715 \\
\hline $\mathrm{H}$ & 1.980835408873 \\
\hline $\mathrm{H}$ & 1.204817769603 \\
\hline $\mathrm{H}$ & 1.110842077692 \\
\hline $\mathbf{C}$ & 2.665093608818 \\
\hline H & 2.128709538897 \\
\hline $\mathrm{H}$ & 3.550827975481 \\
\hline H & 2.005011998536 \\
\hline C & 3.908975922699 \\
\hline H & 4.248505887806 \\
\hline H & 4.790445075045 \\
\hline H & 3.314754578376 \\
\hline C & 2.871521276616 \\
\hline C & 3.027721107962 \\
\hline C & 1.917387171957 \\
\hline C & 2.262537295391 \\
\hline C & 1.132851012908 \\
\hline C & 1.313693086036 \\
\hline H & 2.411811402378 \\
\hline $\mathrm{H}$ & 0.388579096087 \\
\hline H & 0.708963348918 \\
\hline C & 8.200952148307 \\
\hline C & 7.465110358086 \\
\hline C & 9.465832775424 \\
\hline C & 7.970851288056 \\
\hline C & 9.991870672808 \\
\hline C & 9.244945576550 \\
\hline H & 7.376719110375 \\
\hline H & 10.978099638828 \\
\hline H & 9.654972978918 \\
\hline 0 & 3.976353828247 \\
\hline 0 & 6.204267529856 \\
\hline H & 10.037582644415 \\
\hline H & 1.782161998006 \\
\hline Rh & 6.170648920723 \\
\hline C & 4.681035844211 \\
\hline $\mathrm{H}$ & 4.375131437487 \\
\hline $\mathrm{H}$ & 3.779842144171 \\
\hline C & 5.626755570987 \\
\hline H & 5.189270994826 \\
\hline 0 & 6.854335802448 \\
\hline C & 6.844392384968 \\
\hline 0 & 5.948250607546 \\
\hline
\end{tabular}

0.716553730138

2. 026367340362

2. 169551316478

2. 047117485159

2. 866483454139

1. 198639547817

4. 018992228063

3. 846497816079

4. 430337953448

4. 782024106059

3. 275608906073

3. 927247905582

4. 724376106489

4. 382022150406

3. 199547145360

2. 183574406086

2. 638655755243

1.673932038155

1.426249347135

4. 347826116942

5. 122483450207

3. 919222225507

4.839264121617

1.776628869691

2. 418669428878

0.759596200297

2. 073410337095

0.408974084222

1.060263129985

2. 592189060527

$-0.375842088994$

0.777548273708

4.068887312729

5.242484447292

3. 948310847685

6.304272889426

5.005163797718

6.174016263925

7.202039000622

4.914685953904

6.992263111639

3. 400966537443

5. 305596143131

3.032304663728

0.240723527316

1.139685695317

$-1.402744590399$

$-2.020128209444$

$-0.981664156786$

$-0.293616005578$

0.237364341607

$-0.941463879675$

$-1.446902300775$

$-1.286276365356$
1. 261501988482

0.984015580585

2. 901046483038

3. 869680223202

3.049178746815

2. 627640934992

2. 360229164007

3. 312379074476

1. 650797511522

2. 544977643481

$-2.321685945328$

$-1.764418450149$

$-1.044340129020$

$-2.592164171931$

$-1.275541931716$

$-3.342368127761$

$-4.185112785957$

$-3.735340562872$

$-2.904143637960$

$-3.010733811522$

$-2.315279058200$

$-3.493176214589$

$-3.791894217326$

0.250431019572

1.486298527882

0.134369180401

2. 598781472154

1.234835875274

2. 459750254887

3.540013514663

1.139891838686

3. 316795713841

$-0.766271358270$

$-0.976799976164$

$-1.351034752055$

$-1.724454123418$

$-2.098041799390$

$-2.279970018200$

$-1.861811348889$

$-2.543329650130$

$-2.865463275105$

1.576269259209

$-0.446209647228$

$-1.231447186253$

$-0.809773828621$

$-1.416338718079$

$-0.342815981936$

0.513148222983

$-0.794047483968$

0.106697298777

0.952953865295

0.597527096922

1.848483961265

2.653812574703 


$\begin{array}{lrrr}\mathrm{C} & 8.097287896315 & -2.255577448892 & 2.117022229937 \\ \mathrm{H} & 8.203099469947 & -2.417761372295 & 3.191050232303 \\ \mathrm{H} & 8.985399137992 & -1.760151126637 & 1.715210741772 \\ \mathrm{H} & 8.011193375451 & -3.227584734846 & 1.617284156761 \\ \mathrm{H} & 5.153326386750 & -2.052175322360 & -1.086592214729 \\ \mathrm{C} & 6.698746702829 & 2.209628151694 & -2.958022343240 \\ \mathrm{O} & 7.071283280908 & 2.710269991129 & -3.926844369403 \\ \mathrm{C} & 6.921587329920 & -0.409035383842 & -2.232341237569 \\ \mathrm{O} & 7.399000917449 & -1.334991876315 & -2.732715860002\end{array}$

SCF Done: E (UB3LYP) $=-2331.65997241$

gCP correction=

D3 correction=

DFT-gCP-D3 E (UB3LYP) $=-2331.69721563$

Zero-point correction=

Thermal correction to Energy=

Thermal correction to Enthalpy=

Thermal correction to Gibbs Free Energy=

Sum of DFT-gCP-D3 and zero-point Energies=

Sum of DFT-gCP-D3 and thermal Energies=

Sum of DFT-GCP-D3 and thermal Enthalpies=

Sum of DFT-gCP-D3 and thermal Free Energies=

0.173003

$-0.210247$

$\underline{\text { TS-B-1 }}$

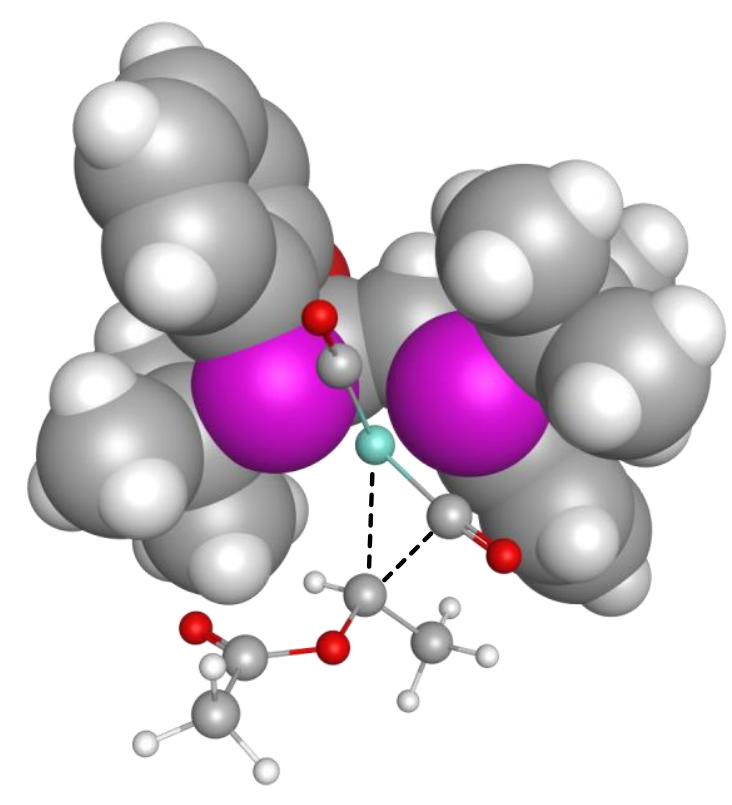

$\begin{array}{lrrr}\mathrm{P} & 1.144015037696 & -0.999811400024 & -0.848391700440 \\ \mathrm{P} & 4.176785021623 & -0.620925347381 & 0.378839855366 \\ \mathrm{C} & 1.571968073384 & 0.649916321746 & -0.015058029585 \\ \mathrm{H} & 1.699725028277 & 1.421258434643 & -0.778908071250 \\ \mathrm{C} & 2.845895048790 & 0.650501896358 & 0.858249131950 \\ \mathrm{H} & 2.544093647880 & 0.506066302728 & 1.898644143428 \\ \mathrm{C} & 5.179560288197 & -0.857357325281 & 1.999735655060 \\ \mathrm{C} & 6.520631299841 & -1.506537019138 & 1.604764630586 \\ \mathrm{H} & 7.108329525109 & -1.692922853250 & 2.513056827770\end{array}$




\begin{tabular}{|c|c|c|c|}
\hline $\mathrm{H}$ & 6.375340087845 & -2.467716476439 & 1.105071357037 \\
\hline $\mathrm{H}$ & 7.111792480748 & -0.845230279872 & 0.961114849718 \\
\hline C & 4.396648978283 & -1.799144472885 & 2.935422819056 \\
\hline $\mathrm{H}$ & 4.889793497903 & -1.819920322729 & 3.915904004978 \\
\hline H & 3.363620298346 & -1.467880424396 & 3.10087625222 \\
\hline H & 4.390898693139 & -2.822851011041 & 2.55688298322 \\
\hline C & 5.452150300620 & 0.475830479369 & 2.72287939191 \\
\hline H & 6.058469478255 & 0.267131559970 & 3.613894716341 \\
\hline H & 6.013350600785 & 1.178249260714 & 2.100069747686 \\
\hline H & 4.536300083116 & 0.972803504442 & 3.05797896347 \\
\hline C & 0.276058109009 & -0.469076442192 & -2.47646123877 \\
\hline C & -0.822338932083 & 0.585939302326 & -2.25297705832 \\
\hline H & -0.429826628790 & 1.513775100496 & -1.82331461342 \\
\hline $\mathrm{H}$ & -1.280678477271 & 0.838868098962 & -3.21807495379 \\
\hline $\mathrm{H}$ & -1.614960760059 & 0.217318073960 & -1.59509828517 \\
\hline C & -0.349542160058 & -1.743680908531 & -3.07618893070 \\
\hline $\mathrm{H}$ & -0.823337472074 & -1.502650626853 & -4.03660152323 \\
\hline $\mathrm{H}$ & 0.399687206147 & -2.520459818320 & -3.25854464966 \\
\hline $\mathrm{H}$ & -1.125647254795 & -2.158756897039 & -2.42302690113 \\
\hline C & 1.344317689781 & 0.084221313866 & -3.43635418049 \\
\hline $\mathrm{H}$ & 1.815301345998 & 0.996917768764 & -3.05145350443 \\
\hline H & 2.134847150481 & -0.646259808967 & -3.62889898909 \\
\hline H & 0.873444829628 & 0.339938334269 & -4.39430531682 \\
\hline C & -0.248497588934 & -1.159076860857 & 0.32734961599 \\
\hline $\mathrm{C}$ & -0.405074617703 & 0.024003694169 & 1.06634473801 \\
\hline C & -1.122049865214 & -2.226973447414 & 0.55845726272 \\
\hline C & -1.413864947677 & 0.163959743900 & 2.01941101028 \\
\hline C & -2.138931849382 & -2.102103394316 & 1.50820879625 \\
\hline C & -2.278752207566 & -0.911216707263 & 2.23015553180 \\
\hline H & -1.510191338145 & 1.092294302910 & 2.57324460186 \\
\hline H & -2.818075415546 & -2.930237904783 & 1.68731355425 \\
\hline H & -3.067919925522 & -0.819127065452 & 2.97138607097 \\
\hline C & 5.070410942762 & 0.742158536038 & -0.45209604108 \\
\hline C & 4.516208473529 & 1.976956775814 & -0.08026905017 \\
\hline C & 6.173277304006 & 0.719065963291 & -1.31053339276 \\
\hline C & 5.041674727072 & 3.183668142643 & -0.54081775163 \\
\hline C & 6.710456225278 & 1.919603739010 & -1.78153575492 \\
\hline C & 6.145672011980 & 3.139832734025 & -1.39391537526 \\
\hline H & 4.589858649942 & 4.122289024813 & -0.23658911299 \\
\hline $\mathrm{H}$ & 7.564522742325 & 1.903445557467 & -2.45172723926 \\
\hline $\mathrm{H}$ & 6.564797374650 & 4.070620813751 & -1.76639307742 \\
\hline 0 & 0.452933248057 & 1.055950524380 & 0.80500151731 \\
\hline 0 & 3.448735680865 & 1.961267982504 & 0.77642857650 \\
\hline H & 6.607813913080 & -0.227163587989 & -1.61689341397 \\
\hline $\mathrm{H}$ & -1.010436043587 & -3.152077257002 & 0.00010471186 \\
\hline $\mathrm{Rh}$ & 3.172383340535 & -2.367974831097 & -1.04773419788 \\
\hline C & 1.611888470451 & -4.867183960011 & 0.84047109318 \\
\hline H & 1.750841557070 & -5.576669492782 & 1.66979068639 \\
\hline $\mathrm{H}$ & 0.973818666992 & -4.052520736762 & 1.19452966256 \\
\hline C & 2.949591616758 & -4.315820881049 & 0.39351574693 \\
\hline H & 3.442116958666 & -3.770171669828 & 1.18775356853 \\
\hline 0 & 3.788967464725 & -5.421764254967 & 0.01327675021 \\
\hline C & 5.052025381102 & -5.470446429109 & 0.47582390294 \\
\hline 0 & 5.526229605394 & -4.679245758782 & 1.26924154885 \\
\hline
\end{tabular}




$\begin{array}{ll}\mathrm{C} & 5.812003535786 \\ \mathrm{H} & 6.668962813697 \\ \mathrm{H} & 6.177319501178 \\ \mathrm{H} & 5.166694101972 \\ \mathrm{H} & 1.088749281323 \\ \mathrm{C} & 4.363511866157 \\ \mathrm{O} & 5.044340623954 \\ \mathrm{C} & 2.445904956776 \\ \mathrm{O} & 1.960609887445\end{array}$

$-6.618161235259$

$-6.871653139326$

$-6.310016263619$

$-7.488873111204$

$-5.397975872427$

$-1.949564663633$

$-1.803022250050$

$-3.930188002773$

$-4.822173995236$
$-0.147300352362$

0.479213767585

$-1.134656282996$

$-0.288606332284$

0.037832916519

$-2.490709005443$

$-3.416366651836$

$-1.769952409403$

$-2.335286573316$

SCF Done: $E$ (UB3LYP) $=-2331.64363314$

gCP correction=

D3 correction=

DFT-gCP-D3 E (UB3LYP) $=-2331.67739100$

Zero-point correction=

0.172357

$-0.206115$

Thermal correction to Energy=

Thermal correction to Enthalpy=

Thermal correction to Gibbs Free Energy=

Sum of DFT-gCP-D3 and zero-point Energies=

Sum of DFT-gCP-D3 and thermal Energies=

Sum of DFT-GCP-D3 and thermal Enthalpies=

Sum of DFT-gCP-D3 and thermal Free Energies= TS-B-1-PDT

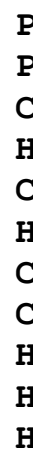

1.849538649733

4. 864795547167

2. 223080567140

2. 296807287458

3. 521714236312

3. 280312191479

5. 919835706613

7. 180067046928

7. 816288412235

6.925499985967

7. 774187120563

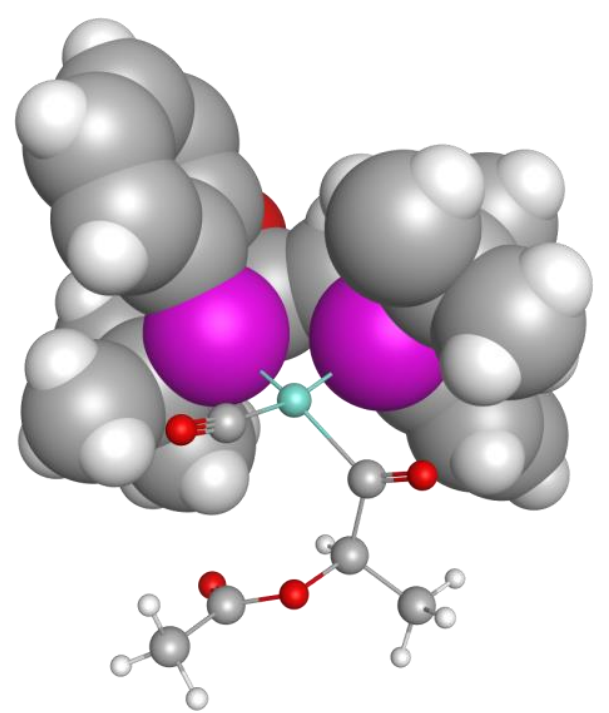

0.579575

0.619733

0.620677

0.506400

$-2331.097816$

$-2331.057658$

$-2331.056714$

$-2331.170991$
$-0.703771096901$

0.261179112405

0.051836080478

$-0.746298997992$

0.875821101721

1.924088852412

1.810088935665

1. 330771506634

2. 195215738031

0.845743615019

0.635876351962 


\begin{tabular}{|c|c|c|c|}
\hline C & 5.093315817378 & -1.910499388194 & 2.728245730496 \\
\hline $\mathrm{H}$ & 5.696479191255 & -2.179817536828 & 3. 605080717261 \\
\hline $\mathrm{H}$ & 4.186742574781 & -1.417282307814 & 3.100430829300 \\
\hline $\mathrm{H}$ & 4.810615328142 & -2.841101939501 & 2.226542797141 \\
\hline C & 6.328603568539 & 0.289468932167 & 2.560811743402 \\
\hline $\mathrm{H}$ & 6.923028585433 & 0.013727542472 & 3.441610469294 \\
\hline $\mathrm{H}$ & 6.941293775000 & 0.949805794969 & 1.939108541468 \\
\hline $\mathrm{H}$ & 5.464231969430 & 0.862078719745 & 2.912420047286 \\
\hline C & 1.053033253521 & -0.779284703168 & -2.385670166083 \\
\hline C & 0.028654400994 & 0.363699280759 & -2.267057676630 \\
\hline $\mathrm{H}$ & 0.473043133124 & 1.306234811745 & -1.930757776252 \\
\hline $\mathrm{H}$ & -0.410817378637 & 0.546198463816 & -3.256208468993 \\
\hline $\mathrm{H}$ & -0.788578048008 & 0.109314884730 & -1.584539704084 \\
\hline C & 0.346522489207 & -2.043000260644 & -2.912532890676 \\
\hline $\mathrm{H}$ & -0.080310628302 & -1.825305546123 & -3.900573725769 \\
\hline $\mathrm{H}$ & 1.033460344545 & -2.887223235800 & -3.009509236714 \\
\hline $\mathrm{H}$ & -0.481479170288 & -2.340996578761 & -2.258637427246 \\
\hline C & 2.194266131707 & -0.383646154100 & -3.341523548230 \\
\hline $\mathrm{H}$ & 2.730380981310 & 0.511458225567 & -3.002311587011 \\
\hline $\mathrm{H}$ & 2.921486451263 & -1.195093385979 & -3.447033311517 \\
\hline $\mathrm{H}$ & 1.777084439073 & -0.160537935401 & -4.332006785157 \\
\hline C & 0.458339019216 & -1.372915066722 & 0.461513511766 \\
\hline C & 0.269331223792 & -0.171901904893 & 1.162296687925 \\
\hline C & -0.374340208808 & -2.464265366856 & 0.728767930350 \\
\hline C & -0.745042550874 & -0.035019052483 & 2.110438237348 \\
\hline C & -1.393692646923 & -2.341717148069 & 1.674700799910 \\
\hline C & -1.573107571363 & -1.131225853431 & 2.355443505884 \\
\hline $\mathrm{H}$ & -0.870342919513 & 0.905455429018 & 2.637147879002 \\
\hline $\mathrm{H}$ & -2.044468322031 & -3.185303940945 & 1.884001369638 \\
\hline $\mathrm{H}$ & -2.365033926547 & -1.041232886496 & 3.094001836501 \\
\hline C & 5.673452068763 & 0.809845369837 & -0.530528026966 \\
\hline C & 5.083563458627 & 2.005121044593 & -0.087742840797 \\
\hline C & 6.730490178412 & 0.863213559779 & -1.444224130931 \\
\hline C & 5.541367612048 & 3.248605913074 & -0.522092564404 \\
\hline C & 7.198919876316 & 2.101737431539 & -1.888729915533 \\
\hline C & 6.606915672096 & 3.281969920485 & -1.423405526433 \\
\hline $\mathrm{H}$ & 5.067328434203 & 4.156315052517 & -0.162823237931 \\
\hline $\mathrm{H}$ & 8.019019630270 & 2.148048175815 & -2.598868491060 \\
\hline $\mathrm{H}$ & 6.972418877160 & 4.242848908863 & -1.775316805919 \\
\hline 0 & 1.103261485786 & 0.874141724807 & 0.878922064785 \\
\hline 0 & 4.048588226844 & 1.911446555147 & 0.803577682670 \\
\hline $\mathrm{H}$ & 7.182396645853 & -0.054938990081 & -1.809661907459 \\
\hline $\mathrm{H}$ & -0.228041076922 & -3.401004265107 & 0.197852350502 \\
\hline $\mathrm{Rh}$ & 3.834016092657 & -2.529507273266 & -0.784108215215 \\
\hline C & 1.821944956427 & -6.299748157451 & 0.043113803611 \\
\hline $\mathrm{H}$ & 1.997386668201 & -7.042138583829 & 0.828525870232 \\
\hline $\mathrm{H}$ & 0.880391569522 & -5.783554761496 & 0.261722858091 \\
\hline C & 2.965779558504 & -5.298117194026 & -0.002098151867 \\
\hline $\mathrm{H}$ & 3.079977167424 & -4.766937205716 & 0.944872171858 \\
\hline 0 & 4.193632865090 & -6.028633275853 & -0.257925656144 \\
\hline C & 5.221347623579 & -5.902830625976 & 0.612493105460 \\
\hline 0 & 5.192675402573 & -5.232538325212 & 1.624410665712 \\
\hline C & 6.414821443822 & -6.696989037643 & 0.134441897546 \\
\hline $\mathrm{H}$ & 7.147582611620 & -6.774544551347 & 0.938909751211 \\
\hline
\end{tabular}




$\begin{array}{llll}\mathrm{H} & 6.867171379039 & -6.181101045101 & -0.720322105181 \\ \mathrm{H} & 6.110385132834 & -7.691185943194 & -0.204200428526 \\ \mathrm{H} & 1.718318900507 & -6.803217309363 & -0.920952346821 \\ \mathrm{C} & 5.378272098750 & -3.285378934713 & -1.584637683367 \\ \mathrm{O} & 6.320152309336 & -3.631195879897 & -2.162420975709 \\ \mathrm{C} & 2.830781926921 & -4.271507708280 & -1.187378498805 \\ \mathrm{O} & 2.183199625730 & -4.604779993533 & -2.159115241652\end{array}$

SCF Done: $E$ (UB3LYP) $=-2331.680419$

gCP correction=

D3 correction=

DFT-gCP-D3 E (UB3LYP) $=-2331.710981$

Zero-point correction=

581826

Thermal correction to Energy=

Thermal correction to Enthalpy=

Thermal correction to Gibbs Free Energy=

Sum of DFT-gCP-D3 and zero-point Energies=

Sum of DFT-gCP-D3 and thermal Energies=

Sum of DFT-gCP-D3 and thermal Enthalpies=

Sum of DFT-gCP-D3 and thermal Free Energies=

0.172937

$-0.203499$

$\underline{\text { TS-D-1-SM }}$

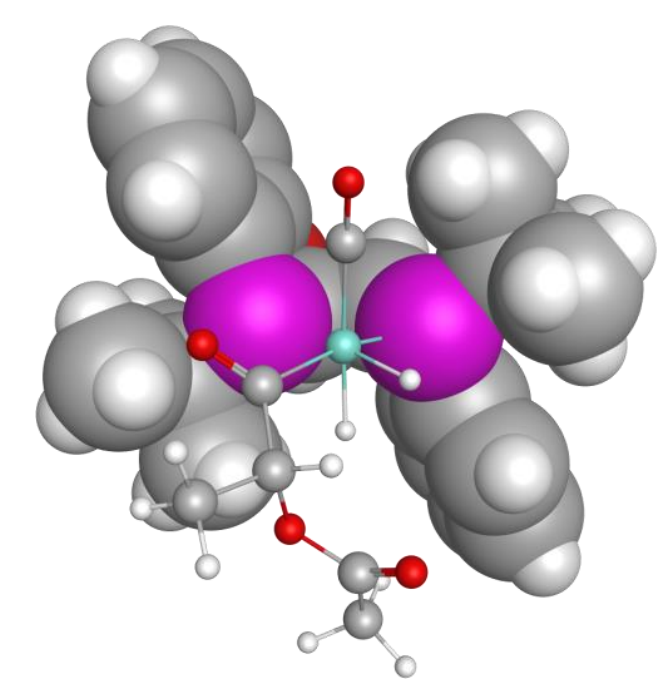

$\begin{array}{lrrr}\text { P } & 0.077145694945 & 0.900884828732 & 0.925918617269 \\ \text { P } & 1.893670272492 & 3.156149446548 & -0.590806324612 \\ \text { C } & 1.670624785483 & 0.292385900335 & 0.090582006795 \\ \text { H } & 2.392780593950 & -0.032580721915 & 0.843615962441 \\ \text { C } & 2.384732762710 & 1.326318192465 & -0.816523390454 \\ \text { H } & 2.202314687589 & 1.021402431890 & -1.849756802397 \\ \text { C } & 2.070772700480 & 3.887233747979 & -2.350168336565 \\ \text { C } & 2.178615903605 & 5.419271445320 & -2.212924416009 \\ \text { H } & 2.222516785249 & 5.864247761384 & -3.215600584563 \\ \text { H } & 1.328100556365 & 5.853415338148 & -1.683426227006 \\ \text { H } & 3.092618059357 & 5.710451275130 & -1.683802700009 \\ \text { C } & 0.804615265828 & 3.516125454581 & -3.143915502428\end{array}$




\begin{tabular}{|c|c|c|c|}
\hline $\mathrm{H}$ & 0.878837179708 & 3.933353332037 & -4.156625074996 \\
\hline $\mathrm{H}$ & 0.686890214107 & 2.429934543087 & -3.246201802165 \\
\hline H & -0.100693505103 & 3.910598958136 & -2.674136586699 \\
\hline C & 3.324752308854 & 3. 365235035614 & -3.077115058260 \\
\hline $\mathrm{H}$ & 3.389606820188 & 3.849959534286 & -4.05987070078 \\
\hline $\mathrm{H}$ & 4.242977707477 & 3.602721865688 & -2.53086432295 \\
\hline H & 3.299320305102 & 2.284523788952 & -3.24961843224 \\
\hline C & 0.026470926036 & -0.040311545646 & 2.58749547478 \\
\hline C & 0.240920472404 & -1.554732509725 & 2.41384028734 \\
\hline H & 1.214839745449 & -1.791794006627 & 1.97246647792 \\
\hline $\mathrm{H}$ & 0.197185063742 & -2.040012822084 & 3. 39763646158 \\
\hline $\mathrm{H}$ & -0.533773274418 & -2.005240103447 & 1.78632091232 \\
\hline C & -1.363482497965 & 0.220507545982 & 3.20222672606 \\
\hline $\mathrm{H}$ & -1.423955548724 & -0.263143353269 & 4.18563679839 \\
\hline $\mathrm{H}$ & -1.556427065322 & 1.289941110325 & 3.33585420865 \\
\hline $\mathrm{H}$ & -2.164432158735 & -0.195697616299 & 2.58075312527 \\
\hline C & 1.118645595574 & 0.540487558955 & 3.50460171884 \\
\hline $\mathrm{H}$ & 2.126436036587 & 0.393149539731 & 3.09796041028 \\
\hline $\mathrm{H}$ & 0.978045131408 & 1.609350550458 & 3.68584605341 \\
\hline H & 1.083885265954 & 0.032626986792 & 4.47679086890 \\
\hline C & -0.868974145069 & -0.143605270911 & -0.22439664993 \\
\hline C & -0.003599675447 & -0.976470994669 & -0.9474862929 \\
\hline C & -2.245005972964 & -0.203828904607 & -0.46415047262 \\
\hline C & -0.485575002920 & -1.888609073587 & -1.88667522579 \\
\hline C & -2.742425791250 & -1.112464374748 & -1.40120293149 \\
\hline C & -1.863377305200 & -1.948670024435 & -2.10076003367 \\
\hline H & 0.206171415951 & -2.522439952811 & -2.43207980746 \\
\hline $\mathrm{H}$ & -3.810391910426 & -1.166599354673 & -1.58982422261 \\
\hline $\mathrm{H}$ & -2.254902201916 & -2.650693019698 & -2.83197085547 \\
\hline C & 3.506539697601 & 3.441929276271 & 0.19776176976 \\
\hline C & 4.324079383110 & 2.311103502225 & 0.06540104817 \\
\hline C & 3.977859897537 & 4.563222273288 & 0.88776730371 \\
\hline C & 5.618548452289 & 2.285842716878 & 0.58438248461 \\
\hline C & 5.270987756366 & 4.550805461483 & 1.41485427885 \\
\hline C & 6.080740509656 & 3.419261542964 & 1.25633236142 \\
\hline $\mathrm{H}$ & 6.234119264261 & 1.399750357438 & 0.46761454287 \\
\hline $\mathrm{H}$ & 5.646322191489 & 5.417046826156 & 1.95118799187 \\
\hline $\mathrm{H}$ & 7.084867681399 & 3.413931673328 & 1.67171076473 \\
\hline 0 & 1.338354427871 & -0.875121596049 & -0.69657174799 \\
\hline 0 & 3.811805719224 & 1.229979790077 & -0.60022290449 \\
\hline H & 3. 335190158496 & 5.430676978597 & 1.01223671773 \\
\hline $\mathrm{H}$ & -2.918681306754 & 0.460169473495 & 0.06998466739 \\
\hline $\mathrm{Rh}$ & -0.166667848265 & 3.310605911529 & 0.71959367949 \\
\hline C & -2.135626035238 & 7.148611339785 & -0.65319993914 \\
\hline $\mathrm{H}$ & -3.167642100501 & 7.386814728933 & -0.92993079070 \\
\hline H & -1.483397853990 & 7.353273432060 & -1.50768102705 \\
\hline C & -2.036785695925 & 5.688397209488 & -0.23163696570 \\
\hline H & -2.728170873819 & 5.467408492611 & 0.58808131380 \\
\hline 0 & -2.403680923621 & 4.861082415202 & -1.35538823862 \\
\hline C & -3.550210112523 & 4.141751050399 & -1.26853757934 \\
\hline 0 & -4.317806177933 & 4.184490782572 & -0.33271544524 \\
\hline C & -3.724017581933 & 3.272740247467 & -2.49224777698 \\
\hline H & -3.413921238555 & 3.800046828325 & -3.39833092041 \\
\hline $\mathrm{H}$ & -3.090238344218 & 2.384373610265 & -2.3870368280 \\
\hline
\end{tabular}




$\begin{array}{lr}\mathrm{H} & -4.766303066425 \\ \mathrm{H} & -1.829070802520 \\ \mathrm{C} & -0.617113370944 \\ \mathrm{O} & 0.197803466010 \\ \mathrm{H} & -0.987923909167 \\ \mathrm{C} & 0.532113000727 \\ \mathrm{O} & 0.832917604745 \\ \mathrm{H} & -1.643449229587\end{array}$

2.958883398498

7.798618237481

5.282545354987

6.169885536254

2. 951456791231

3. 947659470446

4. 382470037381

3. 244058005531
$-2.570367175276$

0.170317377082

0.257744088003

0.434537878453

$-0.602222847983$

2.463379202243

3.482713518006

1.318106842631

SCF Done: $E$ (UB3LYP) $=-2332.85310909$

gCP correction=

D3 correction=

DFT-gCP-D3 E (UB3LYP) $=-2332.88582994$

Zero-point correction=

0.176371

$-0.209092$

Thermal correction to Energy=

Thermal correction to Enthalpy=

Thermal correction to Gibbs Free Energy=

Sum of DFT-gCP-D3 and zero-point Energies=

Sum of DFT-gCP-D3 and thermal Energies=

Sum of DFT-gCP-D3 and thermal Enthalpies=

Sum of DFT-gCP-D3 and thermal Free Energies=

0.598128

0.638877

0.639821

0.523406

$-2332.287702$

$-2332.246953$

$-2332.246009$

$-2332.362424$

$\underline{\text { TS-D-1 }}$

$\begin{array}{ll}\text { P } & 0.003777376736 \\ \text { P } & 2.143133214710 \\ \text { C } & 1.683219362303 \\ \text { H } & 2.263888027195 \\ \text { C } & 2.561470217935 \\ \text { H } & 2.503975659352 \\ \text { C } & 2.694426117687 \\ \text { C } & 2.852565370333 \\ \text { H } & 3.117232598358 \\ \text { H } & 1.922172707736 \\ \text { H } & 3.659213757088\end{array}$

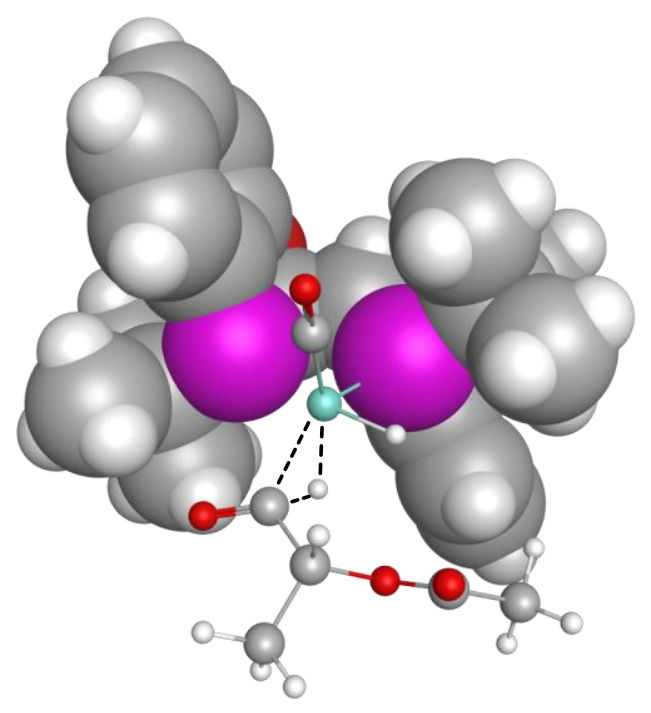
1.181434375090
0.546806230592
3. 308668591374
0.484472595152
0.182038912493
$-0.702757401229$
1.450681800316
0.006956855320
1. 128487323636
0.881591340316
3. 989969347920
$-0.822459724645$
5. 516162178023
$-1.864768774893$
$-2.402312289248$
5.946619458315
$-2.248174219418$
5.981562110963
$-3.223018216366$
5.766886248105
$-1.915211314927$
$-1.549943240146$ 


\begin{tabular}{|c|c|}
\hline C & 1.573778156423 \\
\hline H & 1.856153307218 \\
\hline H & 1.408600144708 \\
\hline H & 0.633781428644 \\
\hline C & 4.028479099526 \\
\hline H & 4.296891613000 \\
\hline H & 4.843796380816 \\
\hline H & 3.974995044199 \\
\hline C & -0.341947510550 \\
\hline C & -0.130166258333 \\
\hline H & 0.893600071663 \\
\hline $\mathrm{H}$ & -0.326027395858 \\
\hline $\mathrm{H}$ & -0.811057396698 \\
\hline C & -1.807295560879 \\
\hline $\mathrm{H}$ & -2.039508699215 \\
\hline $\mathrm{H}$ & -1.992225240492 \\
\hline $\mathrm{H}$ & -2.503922904990 \\
\hline C & 0.596086958348 \\
\hline H & 1.651981843223 \\
\hline H & 0.472348596189 \\
\hline H & 0.371345231040 \\
\hline C & -0.779577670703 \\
\hline C & 0.158558398684 \\
\hline C & -2.095940685663 \\
\hline C & -0.198543183859 \\
\hline C & -2.469231736597 \\
\hline C & -1.523006272672 \\
\hline H & 0.547283343419 \\
\hline H & -3.490762167425 \\
\hline H & -1.816576320384 \\
\hline C & 3.613118357036 \\
\hline C & 4.387559573870 \\
\hline C & 4.034925613867 \\
\hline C & 5.582824703510 \\
\hline C & 5.228162743814 \\
\hline C & 5.993734746736 \\
\hline H & 6.162366458845 \\
\hline H & 5.558590304235 \\
\hline H & 6.919527883445 \\
\hline 0 & 1.443163704368 \\
\hline 0 & 3.938552297734 \\
\hline H & 3.434989539840 \\
\hline H & -2.818161933398 \\
\hline $\mathrm{Rh}$ & -0.107231134714 \\
\hline C & -2.925772751396 \\
\hline $\mathrm{H}$ & -3.889569633897 \\
\hline $\mathrm{H}$ & -3.054598544483 \\
\hline C & -2.393560878210 \\
\hline H & -2.323385186808 \\
\hline 0 & -3.301250322252 \\
\hline C & -4.217508298856 \\
\hline 0 & -4.330022295763 \\
\hline C & -5.058743739686 \\
\hline H & -5.372013165032 \\
\hline
\end{tabular}
3. 672956724280
4. 061437504123
2. 593690842346
4. 146283008112
3. 388886343407
3. 854854539452
3. 585217407723
2. 308339965100
0.309417588437
$-1.213332061138$
$-1.478942145034$
$-1.654494483976$
$-1.683705440788$
0.609945940995
0.151237297238
1.686088203073
0.192276269068
0.922073110999
0.715366110772
2.006198706746
0.487659960146
0.109434705632
$-0.788310310506$
0.084267575721
$-1.732765331404$
$-0.855078737239$
$-1.757993429397$
$-2.416910255702$
$-0.879505711540$
$-2.483820436257$
3. 506803689639
2. 337498446240
4.598839060330
2. 242978410028
4. 518948091957
3. 347759565061
1. 326030001345
5. 364232877537
3. 289062277806
$-0.715159783524$
1.288770888898
5.503634757524
0.803807124931
3. 586584276776
6.697181379829
7.071555598292
6.275775940507
5.643616078149
6.053442328363
4.524405338429
4.496388103250
5. 353586588261
3. 243542257593
3. 054162927251
$-3.410614393044$
$-4.397819590321$
$-3.521037824283$
$-3.116957793626$
$-2.882162336032$
$-3.839146757887$
$-2.178934970896$
$-3.049968437649$
2. 213088719926
2. 137788379739
1.854837931015
3. 123811775768
1. 421901305792
2.584949854523
3.554683689984
2. 666470694989
1. 848316080692
3.269771857613
3. 057507063320
3. 344836130042
4.252169409603
$-0.700882885989$
$-1.231374484983$
$-1.170088001953$
$-2.193623649013$
$-2.133546471709$
$-2.633524145937$
$-2.585279090774$
$-2.500959582183$
$-3.386996933809$
0.358648138909
0.342533697848
1.123099458870
1.055116514745
1.844366652580
1.802918314740
1.023105272299
2. 440301728314
2. 368804556263
$-0.765113030858$
$-0.412904383326$
1.158759905488
$-0.794946479390$
0.327536458631
$-1.446038050607$
$-1.087904276058$
$-2.447992384465$
$-0.481523483172$
0.531802963318
$-0.449672297274$
0.548721744657
1. 396767575628
0.462648339667
$-0.568007875689$ 


$\begin{array}{lr}\mathrm{H} & -4.461099149995 \\ \mathrm{H} & -5.928905308330 \\ \mathrm{H} & -2.222433532892 \\ \mathrm{C} & -0.982054931125 \\ \mathrm{O} & -0.315648884844 \\ \mathrm{H} & -1.004558971870 \\ \mathrm{C} & 0.499338987558 \\ \mathrm{O} & 0.791345677455 \\ \mathrm{H} & -1.509568010980\end{array}$

2. 386053963207

3. 344015464607

7.530570956116

5.134893533409

5.808070675322

3.650291917895

4.457829180085

5.001721586546

3.507552396566
0.793330540901

1.112880735138

$-1.513922334545$

$-0.866418510471$

$-1.631163000902$

$-1.112886040780$

1.929816862596

2.905804291489

1.047844607183

SCF Done: $\mathrm{E}(\mathrm{UB} 3 \mathrm{LYP})=-2332.83474373$

gCP correction=

0.177559

D3 correction=

DFT-gCP-D3 E (UB3LYP) $=-2332.86350072$

Zero-point correction=

$-0.206316$

Thermal correction to Energy=

Thermal correction to Enthalpy=

Thermal correction to Gibbs Free Energy=

Sum of DFT-gCP-D3 and zero-point Energies=

Sum of DFT-gCP-D3 and thermal Energies=

Sum of DFT-gCP-D3 and thermal Enthalpies=

Sum of DFT-gCP-D3 and thermal Free Energies=

$\underline{\mathrm{Rh}-\mathrm{H}-\mathrm{CO}-\mathrm{Ligand}}$

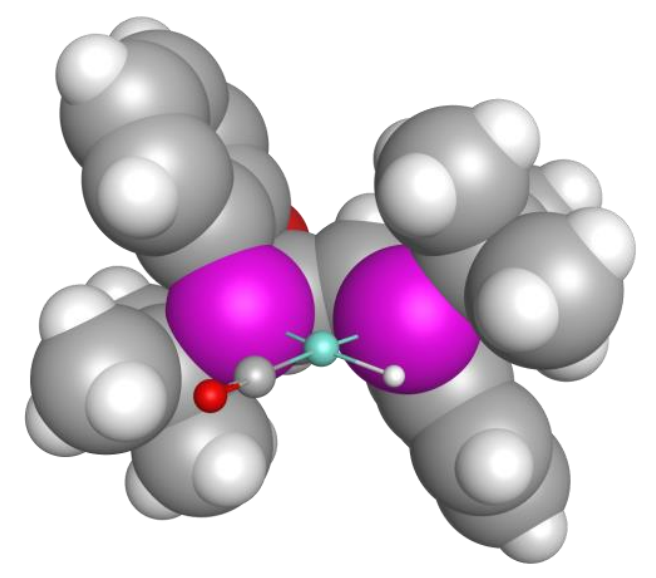

$\begin{array}{ll}\mathrm{P} & -3.552906079539 \\ \mathrm{P} & -1.533185269740 \\ \mathrm{C} & -2.043853291036 \\ \mathrm{H} & -1.322465163246 \\ \mathrm{C} & -1.304771005059 \\ \mathrm{H} & -1.648502711452 \\ \mathrm{C} & -1.399754225171 \\ \mathrm{C} & -1.215347210397 \\ \mathrm{H} & -1.181681469181 \\ \mathrm{H} & -2.039802448396 \\ \mathrm{H} & -0.276314945192 \\ \mathrm{C} & -2.735019851611 \\ \mathrm{H} & -2.734866208406 \\ \mathrm{H} & -2.896397589904\end{array}$

0.176389008119
-2.027009759373
0.829853651337
1.287511665373
-0.255132584030
-0.189498087870
-3.128154186346
-4.577806697276
-5.252909627194
-4.899999842714
-4.701445780269
-3.005063348458
-3.690259393709
-1.993262229358

$-1.023036582729$

0.079691616659

$-0.068736262919$

$-0.750057254624$

0.746325896986

1. 781713533514

1.633185506707

1. 142555647580

2.007382597945

0.498966230592

0.591189768066

2. 392269847790

3. 250105036334

2. 784130658139 


$\begin{array}{lr}\mathrm{H} & -3.585292965159 \\ \mathrm{C} & -0.224605330047 \\ \mathrm{H} & -0.206362420816 \\ \mathrm{H} & 0.737433949558 \\ \mathrm{H} & -0.307005930870 \\ \mathrm{C} & -3.652742778250 \\ \mathrm{C} & -3.454597539258 \\ \mathrm{H} & -2.470564903267 \\ \mathrm{H} & -3.536047883458 \\ \mathrm{H} & -4.216715629203 \\ \mathrm{C} & -5.044629960954 \\ \mathrm{H} & -5.112755764295 \\ \mathrm{H} & -5.223837090460 \\ \mathrm{H} & -5.844727846189 \\ \mathrm{C} & -2.564057475757 \\ \mathrm{H} & -1.553882553227 \\ \mathrm{H} & -2.680037083366 \\ \mathrm{H} & -2.636488305577 \\ \mathrm{C} & -4.617273989707 \\ \mathrm{C} & -3.849883217830 \\ \mathrm{C} & -5.997236172827 \\ \mathrm{C} & -4.438688217244 \\ \mathrm{C} & -6.599160520722 \\ \mathrm{C} & -5.819737633123 \\ \mathrm{H} & -3.823462743158 \\ \mathrm{H} & -7.670260654033 \\ \mathrm{H} & -6.292488313438 \\ \mathrm{C} & 0.172319459001 \\ \mathrm{C} & 0.819642909095 \\ \mathrm{C} & 0.860075705225 \\ \mathrm{C} & 2.145262990752 \\ \mathrm{C} & 2.187563948759 \\ \mathrm{C} & 2.820873674162 \\ \mathrm{H} & 2.621628450660 \\ \mathrm{H} & 2.725644800021 \\ \mathrm{H} & 3.852534226422 \\ \mathrm{O} & -2.500394292111 \\ \mathrm{O} & 0.110767157324 \\ \mathrm{H} & 0.359632683612 \\ \mathrm{C} & -6.591101078975 \\ \mathrm{H} & -3.467230334070 \\ & -3.605546983855 \\ -3.717750013446 \\ -4.833818061569\end{array}$

$-3.257933052211$

$-2.745052904748$

$-3.419493698115$

$-2.837925867478$

$-1.722630036238$

1. 255854590302

2. 750963504704

2. 965991880769

3. 322028251217

3. 129177542148

1.036332320508

1.604230197386

$-0.019595096895$

1. 391279576772

0.752294859399

0.915910411043

$-0.317010812781$

1.298303170510

0.989229182722

1.822027114014

0.893372786627

2.583261770982

1. 648087985229

2. 488433333382

3. 221414183976

1. 578290859755

3. 071067847931

$-1.932167876714$

$-0.789306128510$

$-2.791625231376$

$-0.503297433018$

$-2.518253526156$

$-1.382495155816$

0.386836681321

$-3.183413994331$

$-1.171146113521$

1.876646910857

0.036918480955

$-3.665193616230$

0.228166622813

$-2.142897152832$

$-3.976437222852$

$-5.088449755469$

$-2.004226218264$
1. 749686186582

2. 548753157090

3. 415003261412

2. 035135027756

2. 931588826434

$-2.587459664722$

$-2.281955192346$

$-1.852043674125$

$-3.215765153586$

$-1.593298284025$

$-3.211802449380$

$-4.148691335048$

$-3.435077740034$

$-2.551945726341$

$-3.554666713406$

$-3.159829089715$

$-3.761520524193$

$-4.504181475973$

0.208210621660

1.037208627730

0.412371810349

2.047155539206

1. 421050848134

2. 225634041432

2. 673184643651

1.584984971856

3.011700250398

$-0.563747971153$

$-0.069921717206$

$-1.425629682216$

$-0.396556203681$

$-1.763121990707$

$-1.244551215957$

0.001645468246

$-2.431789937582$

$-1.512645039807$

0.817758402066

0.759615121011

$-1.834281941953$

$-0.207911380561$

$-1.273419739547$

$-1.700128274647$

$-1.995691815970$

$-2.135888111174$

SCF Done: $E$ (UB3LYP) $=-1911.84947629$

gCP correction=

0.130006

D3 correction=

DFT-gCP-D3 E (UB3LYP) $=-1911.88477619$

$-0.165306$

Zero-point correction=

0.470871

0.501320

0.502264

Thermal correction to Energy=

Thermal correction to Enthalpy=

Thermal correction to Gibbs Free Energy=

0.409648 
Sum of DFT-gCP-D3 and zero-point Energies= Sum of DFT-gCP-D3 and thermal Energies= Sum of DFT-GCP-D3 and thermal Enthalpies= Sum of DFT-gCP-D3 and thermal Free Energies=
$-1911.413905$

$-1911.383456$

$-1911.382512$

$-1911.475128$

Formylvinylacetate-PDT

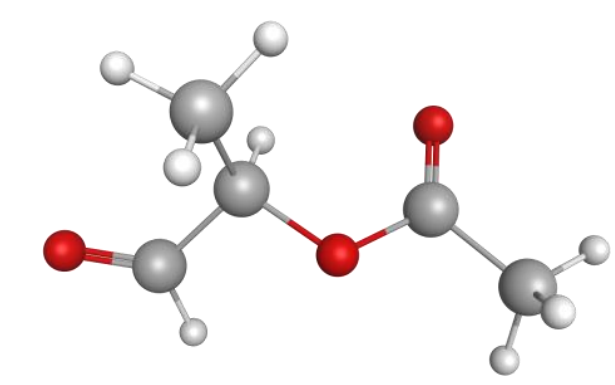

$\begin{array}{llll}\mathrm{C} & -8.386682998023 & -1.391316347285 & 1.474450479472 \\ \mathrm{H} & -9.378533947108 & -1.558154511695 & 1.046237897317 \\ \mathrm{H} & -8.222233984511 & -0.315868028412 & 1.593421041821 \\ \mathrm{C} & -7.317628321163 & -1.998935806242 & 0.577432696196 \\ \mathrm{H} & -7.551613602975 & -3.052555237619 & 0.372379811333 \\ \mathrm{O} & -7.234629976904 & -1.308478790310 & -0.689788934353 \\ \mathrm{C} & -7.976553022730 & -1.813840468491 & -1.713375173031 \\ \mathrm{O} & -8.672358425699 & -2.798542045833 & -1.614475718202 \\ \mathrm{C} & -7.805607518291 & -0.974957919103 & -2.956071426235 \\ \mathrm{H} & -8.172398817678 & 0.040536246403 & -2.772561579947 \\ \mathrm{H} & -6.745710255962 & -0.896476085881 & -3.217877226071 \\ \mathrm{H} & -8.362661876331 & -1.425583155043 & -3.777886967508 \\ \mathrm{H} & -8.338770683589 & -1.858133102213 & 2.462047536471 \\ \mathrm{C} & -5.918619324139 & -1.939791250822 & 1.173872583505 \\ \mathrm{O} & -5.693985104926 & -1.992800606062 & 2.360736858433 \\ \mathrm{H} & -5.102648499973 & -1.867425771390 & 0.422429120802\end{array}$

SCF Done: $\mathrm{E}$ (UB3LYP) $=-421.021196720$

gCP correction=

0.041656 
D3 correction=

DFT-gCP-D3 E (UB3LYP) $=-421.000533055$

Zero-point correction=

Thermal correction to Energy=

Thermal correction to Enthalpy=

Thermal correction to Gibbs Free Energy=

Sum of DFT-gCP-D3 and zero-point Energies=

Sum of DFT-gCP-D3 and thermal Energies=

Sum of DFT-gCP-D3 and thermal Enthalpies=

Sum of DFT-gCP-D3 and thermal Free Energies=
$-0.020992$

0.127405

0.136773

0.137717

0.091763

$-420.893792$

$-420.884424$

$-420.883480$

$-420.929434$

$\underline{\text { Vinylacetate-SM }}$

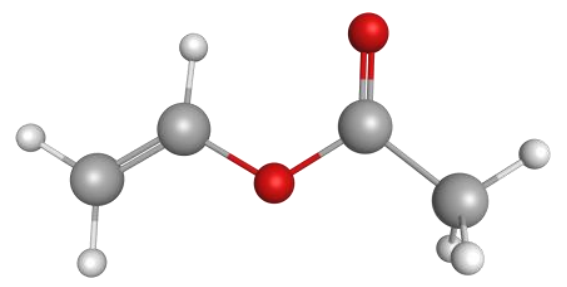

$\begin{array}{lr}\text { C } & 2.076124776253 \\ \mathrm{C} & 0.777421379878 \\ \mathrm{H} & 0.035464626552 \\ \mathrm{O} & 0.269970731445 \\ \mathrm{C} & -1.077113524676 \\ \mathrm{O} & -1.845012642512 \\ \mathrm{C} & -1.431117496888 \\ \mathrm{H} & -1.146890151417 \\ \mathrm{H} & -0.876584656312 \\ \mathrm{H} & -2.503261336470 \\ \mathrm{H} & 2.768475879915 \\ \mathrm{H} & 2.467163601834\end{array}$

2. 162649495047

2. 216454942019

1. 495914486584

3. 258486095269

3. 271433828488

2. 428553436376

4. 475787787802

5.393657187653

4. 455420210381

4. 476773635614

2. 918760373882

1. 344873769209
1.252178471488

0.978881362361

1. 302517617241

0.220071908603

$-0.036034622133$

0.360955561236

$-0.870273142910$

$-0.345092552376$

$-1.814287221875$

$-1.068157923666$

0.896700224370

1.846359320695

SCF Done: E (UB3LYP) $=-306.470702597$

gCP correction=

0.175610

D3 correction=

$-0.199021$

DFT-gCP-D3 E (UB3LYP) $=-306.455863912$

0.094691

Zero-point correction=

0.101518

Thermal correction to Energy=

0.102463 
Thermal correction to Gibbs Free Energy= Sum of DFT-gCP-D3 and zero-point Energies= Sum of DFT-GCP-D3 and thermal Energies= Sum of DFT-gCP-D3 and thermal Enthalpies= Sum of DFT-gCP-D3 and thermal Free Energies=
0.063638

$-306.376012$

$-306.369184$

$-306.368240$

$-306.407064$

Hydrogen

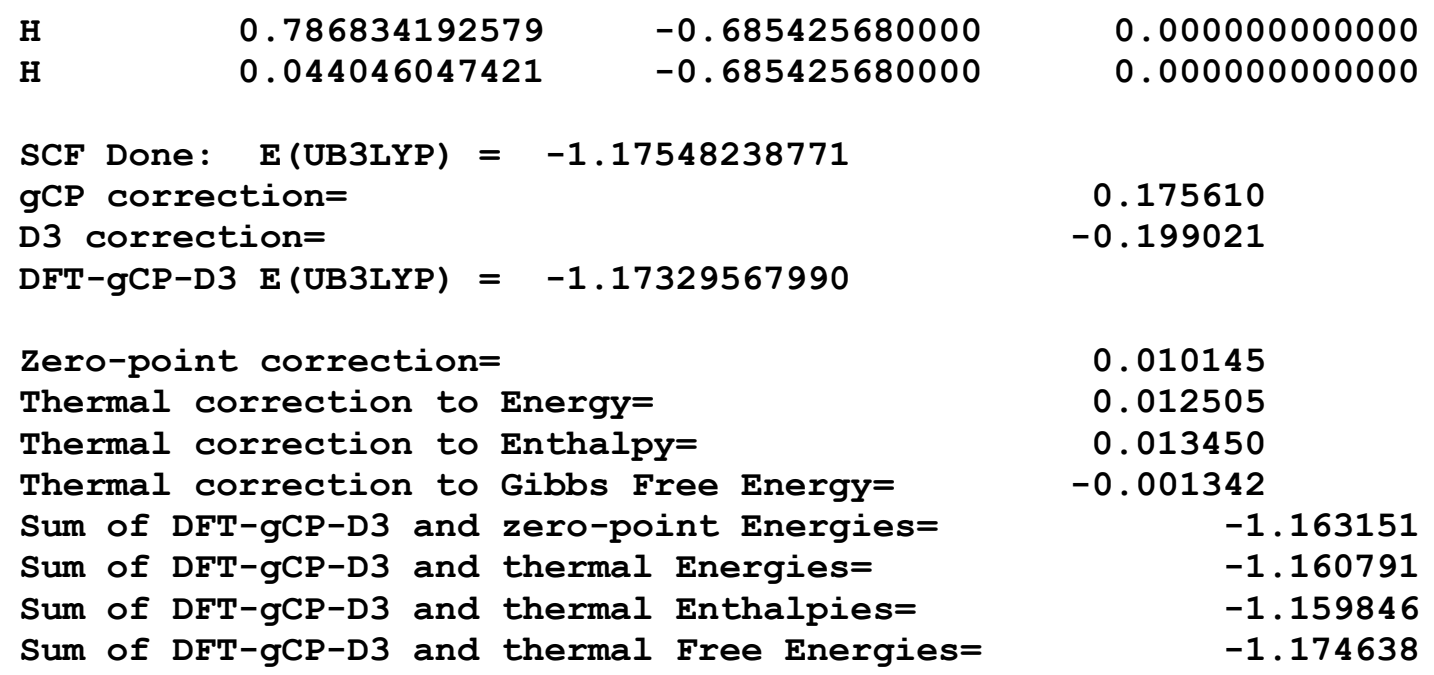

$\underline{\text { Carbon Monoxide }}$

$\begin{array}{lrrr}\text { C } & -1.022561089470 & 0.866529240000 & -0.280562820000 \\ \text { O } & 0.115382229470 & 0.866529240000 & -0.280562820000 \\ & & \\ \text { SCF Done: E (UB3LYP) }= & -113.309454336 & 0.175610 \\ \text { gCP correction= } & & -0.199021 \\ \text { D3 correction= } & \\ \text { DFT-gCP-D3 E (UB3LYP) }= & -113.308550999 & \\ \text { Zero-point correction }= & & 0.005032\end{array}$


Thermal correction to Energy=

Thermal correction to Enthalpy=

Thermal correction to Gibbs Free Energy=

Sum of DFT-gCP-D3 and zero-point Energies=

Sum of DFT-gCP-D3 and thermal Energies=

Sum of DFT-gCP-D3 and thermal Enthalpies=

Sum of DFT-gCP-D3 and thermal Free Energies=
0.007392

0.008337

$-0.014107$

$-113.303519$

$-113.301159$

$-113.300214$

$-113.322658$ 


\section{References}

[1] Tang,W.; Qu, B.; Capacci,A. G.; Rodriguez, S.; Wei, X.; Haddad, N.; Narayanan, B.; Ma, S.; Grinberg, N.; Yee, N.

K; Krishnamurthy, D; Senanayake, C.H.Org. Lett. 2010 , 12, 176.

[2] Zhang, X.; Cao, B.; Yu, S.; Zhang, X. Angew. Chem. Int. Ed. 201 0, 49, 4047.

[3] Xu, K.; Zheng, X.; Wang, Z.; Zhang, X. Chem.Eur.J. 2014, 20, 4357.

[4] Wang, X.; Buchwald, S. L.J. Org. Chem. $2013,78,3429$.

[5] Wang, Q.; Liu, X.; Liu, X.; Li, B.; Nie, H.; Zhang, S.; Chen W. Chem. Commun. 2014 , 50, 978.

[6] Yamada, T.; Sakaguchi, S.; Ishii, Y.J. Org. Chem. 2005, 70, 5471.

[7] Gaussian 09, Revision C.01, Frisch, M.J.; Trucks, G. W.; Schlegel, H. B.; Scuseria, G. E.; Robb, M. A.; Cheeseman, J. R; Scalmani, G.; Barone, V.; Mennucci, B.; Petersson, G. A; Nakatsuji, H.; Caricato, M.; Li, X.; Hratchian, H.P.; Izmaylov, A. F.; Bloino,J.; Zheng, G.; Sonnenberg,J. L.; Hada, M.; Ehara, M.; Toyota, K.; Fukuda, R; Hasegawa,J.; Ishida,M.; Nakajima, T.; Honda, Y.; Kitao, O.; Nakai,H.; Vreven, T.; Montgomery,J.A.,Jr.; Peralta,J.E.; Ogliaro, F.; Bearpark, M.; Heyd,J.J.; Brothers, E.; Kudin, K. N.; Staroverov, V. N.; Kobayashi, R,; Normand,J.; Raghavachari, K.; Rendell, A.; Burant,J. C.; Iyengar, S. S.; Tomasi,J.; Cossi, M.; Rega, N.; Millam, J. M.; Klene, M.; Knox,J.E.; Cross,J. B.; Bakken, V.; Adamo, C.; Jaramillo, J.; Gomperts, R.; Stratmann, R. E.; Yazyev, O.; Austin, A.J.; Cammi, R; Pomelli, C.; Ochterski,J.W.; Martin, R. L.; Morokuma, K; Zakrzewski, V. G.; Voth, G. A.; Salvador, P.; Dannenberg,J.J.; Dapprich, S.; Daniels, A. D.; Farkas, Ö.; Foresman,J.B.; Ortiz,J.V.; Cioslowski,J.; Fox, D.J. Gaussian, Inc, Wallingford CT, 2010.

[8] (a) Becke, A. D.J. Chem. Phys. 1993, 98, 5648-5652.(b) Lee, C.; Yang, W.; Parr, R. G. Phys. Rev. B 1988, 37, 785789.

[9] (a) Hay, P.J.; Wadt, W. R. J. Chem. Phys. 1985, 82, 270-283. (b) Wadt, W. R; Hay, P.J.J. Chem. Phys. 1985, 82, 299-310. (c) Dunning, T.H.Jr.; Hay, P.J.in Modern Theoretical Chemistry, Ed.H. F. Schaefer III, Vol. 3 (Plenum, New York, 1977) 1-28.

[10] (a) Tonks, I. A.; Froese, R. D.; Landis, C. R.ACS Catal. 2013 ,3, 2905-2909. (b) For the DFT computed rhodium catalyzed asymmetric hydroformylation pathway, see: Luo, X.; Tang, D.; Li, M. Int.J. Quantum Chem. 2005, $105,108-123$.

[11] Kruse, H.; Grimme, S.J. Chem. Phys. 2012 , 136, 154101.

[12] Grimme, S.; Ehrlich, S.; Goerigk, L. J. Comput. Chem. 201 1 , 32, 1456-1465.

[13] Kruse, H.; Goerigk, L.; Grimme, S.J. Org. Chem. 2012 , 77, 10824-10834. 